Review

\title{
Artificial Intelligence in Translational Medicine
}

\author{
Simone Brogi *(1) and Vincenzo Calderone *
}

check for

updates

Citation: Brogi, S.; Calderone, V. Artificial Intelligence in Translational Medicine. Int. J. Transl. Med. 2021, 1, 223-285. https://doi.org/10.3390/ ijtm1030016

Academic Editor: Pier Paolo Claudio

Received: 15 October 2021

Accepted: 10 November 2021

Published: 12 November 2021

Publisher's Note: MDPI stays neutral with regard to jurisdictional claims in published maps and institutional affiliations.

Copyright: (c) 2021 by the authors. Licensee MDPI, Basel, Switzerland. This article is an open access article distributed under the terms and conditions of the Creative Commons Attribution (CC BY) license (https:// creativecommons.org/licenses/by/ $4.0 /)$.
Department of Pharmacy, University of Pisa, Via Bonanno 6, 56126 Pisa, Italy

* Correspondence: simone.brogi@unipi.it (S.B.); vincenzo.calderone@unipi.it (V.C.); Tel.: +39-050-2219613 (S.B.); +39-050-2219589 (V.C.)

\begin{abstract}
The huge advancement in Internet web facilities as well as the progress in computing and algorithm development, along with current innovations regarding high-throughput techniques, enable the scientific community to gain access to biological datasets, clinical data and several databases containing billions of pieces of information concerning scientific knowledge. Consequently, during the last decade the system for managing, analyzing, processing and extrapolating information from scientific data has been considerably modified in several fields, including the medical one. As a consequence of the mentioned scenario, scientific vocabulary was enriched by novel lexicons such as machine learning (ML)/deep learning (DL) and overall artificial intelligence (AI). Beyond the terminology, these computational techniques are revolutionizing the scientific research in drug discovery pitch, from the preclinical studies to clinical investigation. Interestingly, between preclinical and clinical research, translational research is benefitting from computer-based approaches, transforming the design and execution of translational research, resulting in breakthroughs for advancing human health. Accordingly, in this review article, we analyze the most advanced applications of AI in translational medicine, providing an up-to-date outlook regarding this emerging field.
\end{abstract}

Keywords: translational medicine; machine learning; artificial intelligence

\section{Introduction}

Nowadays, artificial intelligence (AI), as well as the related specialties of machine learning (ML) and deep learning (DL), are rapidly gaining traction in many sectors, including the scientific (e.g., healthcare), with the potential to transform lives and improve patient outcomes in various fields of medicine. Accordingly, AI companies attracted approximately USD 40 billion worldwide in unveiled investment in 2019 alone [1], reaching USD 232 billion by 2025 [2]. Regarding the scientific areas, these revolutionary computer-based approaches have the potential to revolutionize how clinicians assist patients in clinical practice (precision medicine, virtual diagnosis, and patient monitoring) as well as how scientists discover and deliver new drugs and diagnostic tools [3-5]. These pieces of evidence are also supported by the papers that have been published over the years. In fact, by searching in PubMed the term "artificial intelligence", we obtained over 140,000 published papers in the fields, with a significant increment starting from 2018, testifying that the discipline is of particular interest worldwide (Figure 1, panel A). Furthermore, by adding the term "translational medicine" to "artificial intelligence", we obtained about 2000 publications, with a marked increase from 2019 (Figure 1, panel B). This basic research highlighted the growing interest in AI-based techniques in scientific fields, particularly in translational medicine.

Currently, high-throughput procedures such as parallelized sequencing, microscope imaging, and compound screening are now widely used by academic and biotech/ pharmaceutical researchers, and the number and quality of laboratory data collected have increased dramatically. These "big data" are used for producing biological insights, applying ML techniques, granting a better understanding of disease causes, uncovering new therapy options, and improving diagnostic tools for clinical use [6]. 


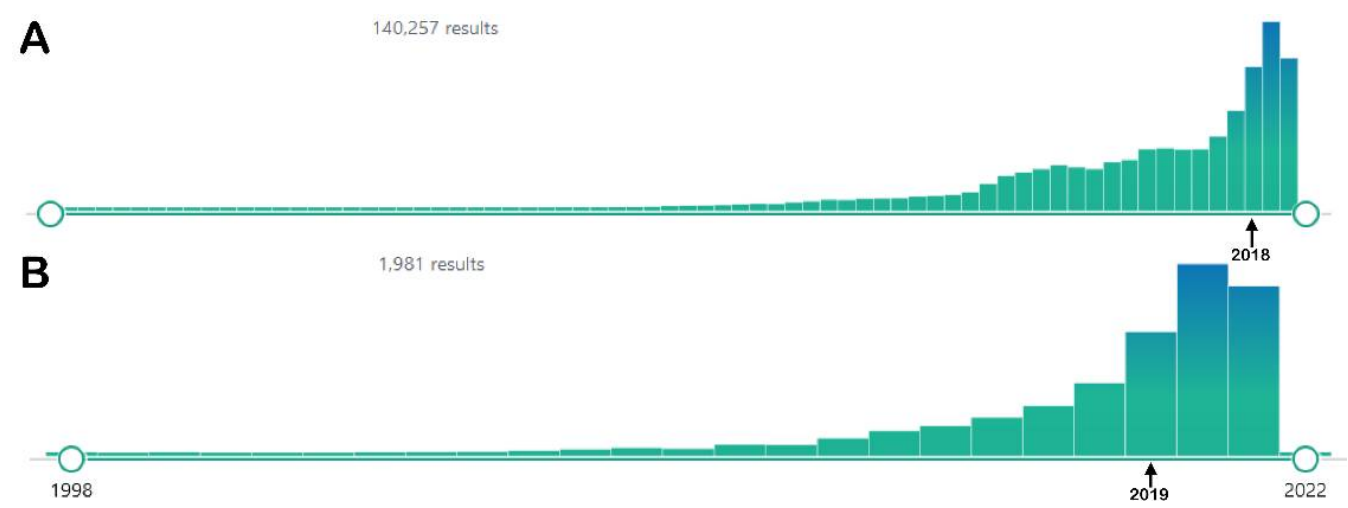

Figure 1. (A) Output of searching the term "artificial intelligence" on PubMed; (B) Output of searching the term "artificial intelligence" and "translational medicine on PubMed. The search was performed on 14 October 2021 (source PubMed https:/ / pubmed.ncbi.nlm.nih.gov/; accessed on 14 October 2021).

In fact, the term AI is defined by US Food and Drug Administration (FDA) as "the science and engineering of making intelligent machines", whereas ML means "an AI technique that can be used to design and train software algorithms to learn from and act on data" [7].

Accordingly, the main goal of these advanced technologies is to analyze the big data employing computer-based algorithms for extracting valuable information to support decision making [8]. Hence, the application of AI methods enables scientists to manage and conduct a broad assortment of tasks, including diagnosis generation and appropriate therapy selection, risk prediction and illness stratification, medical mistake reduction, and productivity improvement, among other things [5,9]. In particular, regarding translational research, a number of high-throughput assays generate data from many patient samples and are acquired into datasets that are in machine-readable format, and hypothetically critical variables are discovered by employing an ML-based algorithm. The algorithm learns relationships between the variables and may perform intelligent tasks, including grouping patients or predicting their outcomes [6]. The role of AI in medicine is summarized in Figure 2. The aspects illustrated in Figure are discussed in Section 2.
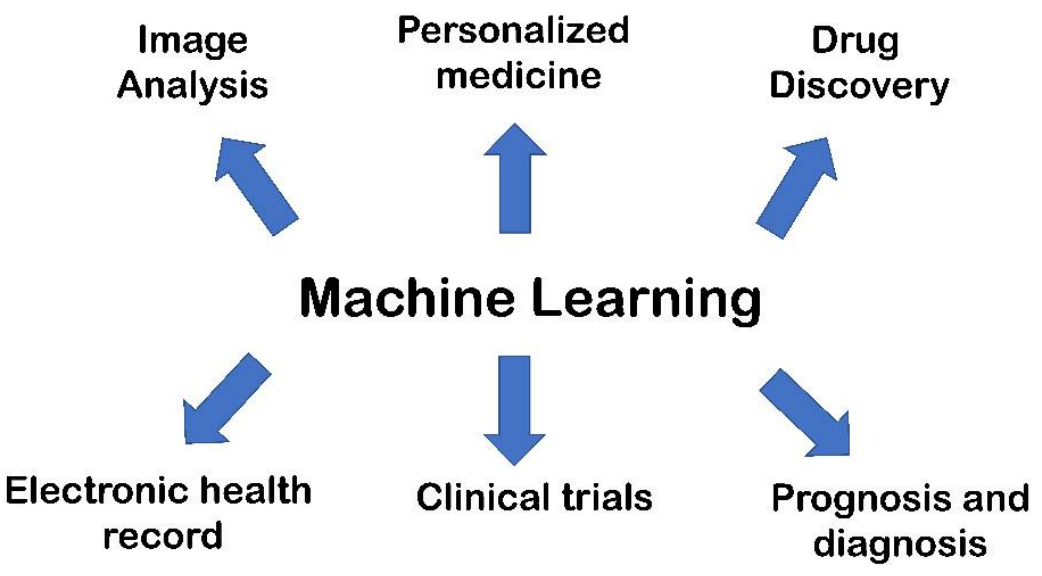

Figure 2. Schematic representation of the application of $\mathrm{AI} / \mathrm{ML}$ in translational medicine.

According to one description, ML is "the fundamental technology required to meaningfully process data that exceed the capacity of the human brain to comprehend" [10]. A large number of data points is used to train ML computer-based models. Existing information about specific data items and relationships between data elements is learned via repeated cycles of mapping between inputs and outputs, rather than being explicitly coded into the model. Therefore, cooperation between ML and clinical specialists is critical, and 
there are many computational approaches that include various degrees of clinical experience into model parameters [11]. Currently, the generation of ML models is mainly grouped into four categories: supervised learning, unsupervised learning, semi-supervised learning, and reinforcement learning. Briefly, the output labels, such as a disorder, are known in advance in supervised ML models. In fact, the objective is to generate a computational tool for predicting an output from a set of input data (i.e., the output is usually termed a target value, response variable, or label, while inputs are termed predictors or features). The method "learns" the best model by analyzing the data contained in the training set, which include many observations, each of which holds values for its characteristics as well as its label. Additionally, there are two kinds of supervised ML: classification and regression. In the classification, the output variable is divided into categories, such as "present" or "absent", "disorder" or "no-disorder", or "grading" (Grade1, Grade2, etc.), while in regression the output variable is an actual value, such as "weight", "dose", or "concentration" (IC 50 , $\mathrm{EC}_{50}, \mathrm{TC}_{50}$, etc.) for performing predictions on novel samples. Such ML may be employed in medical imaging in a variety of areas, including radiology, pathology, and other imaging fields, as well as in epidemiology. Recently, with the outbreak of the COVID-19 pandemic, some ML approaches have been applied for predicting the infection rate, starting from an epidemiology dataset [12], as well as from environmental conditions [13]. Furthermore, in recent years, supervised ML has also been used in drug discovery and development [14-16]. These approaches employing supervised ML are valuable, but they must be approached with prudence because they need huge and reliable data sets containing high-quality data to become accurate, and the data must be correctly categorized [17].

On the other hand, unsupervised ML models aim to identify relationships in data that we would not see otherwise. In particular, there are no labels on the data sets, but they do contain features. As a result, the unsupervised ML algorithm must produce groups and classes based on data set similarities. Unsupervised ML, in contrast to supervised ML, predicts unknown outcomes, uncovering previously undiscovered patterns.

Unsupervised ML is exemplified through clustering. This latter is the process of dividing data into various groups or clusters. Accordingly, when the exact information about the clusters is unknown, we can utilize unsupervised ML to cluster them [18]. Various scientific fields benefit from the application of unsupervised ML. For instance, in a recent report, the unsupervised ML technique was applied for identifying subjects showing a high likelihood of dementia in a population-based survey, with no need of a medical diagnosis of dementia in the subsample [19]. Another study investigated healthcare professionals' feelings toward a digital simulator, technology, and mentality for elucidating their effects on neonatal resuscitation performance in simulation-based assessments [20]. In general pathology, unsupervised ML is becoming a crucial tool for accelerating the transition to autonomous pathological tissue analysis [21]. In another study, an unsupervised ML approach was used to discover patient clusters established by genetic signatures [22]. Additionally, in this case, in drug discovery and development, unsupervised ML has been successfully applied in atomistic simulations or to understand the comportment of chemicals (e.g., drugs) as well as materials [23]. Recently, in a randomized clinical study, unsupervised ML was applied to cluster septic patients to determine optimal treatment (NCT03752489). To further clarify the difference between supervised and unsupervised ML models, a supervised ML model can be used to identify which subjects will develop a given disorder, a known entity, while an unsupervised ML model will be able to identify unknown subgroups of patients suffering from a given pathology since unsupervised models assume that the output labels are unknown. Most of the computer-based models incorporated into clinical workflows, as clinical decision support, are supervised ML models. For improving the performances of ML models, unsupervised and supervised ML can be combined into semi-supervised ML (Figure 3). Ma and colleagues successfully reported a combination of the two strategies for phenotyping complex diseases. They applied this technique to obstructive sleep apnea, highlighting that the phenotyping framework constructed by combining unsupervised and supervised ML techniques can be employed 
for other heterogeneous, complex diseases to phenotype patients, distinguishing significant features for high-risk phenotypes [24]. Omta and coworkers, by combining unsupervised and supervised ML-based tools, showed that they have a great capacity to increase the capability of detecting new knowledge in functional genomics screening. Firstly, they applied unsupervised exploratory ML models to the dataset to gain better insight into the quality of the data. This latter approach enhances the selection and labeling of data for establishing reliable training sets prior to applying ML. For demonstrating the validity of the approach, they used a high-content genome-wide small interfering RNA (siRNA) screen. By applying unsupervised ML models, they easily identified four robust phenotypes that were consequently used as a training set for developing a high-quality random forest (RF) ML tool for differentiating four phenotypes (accuracy $=91.1 \%$; kappa $=0.85$ ). The reported approach significantly improved the ability to obtain novel information from a screening compared with the usage of unsupervised ML techniques alone [25].

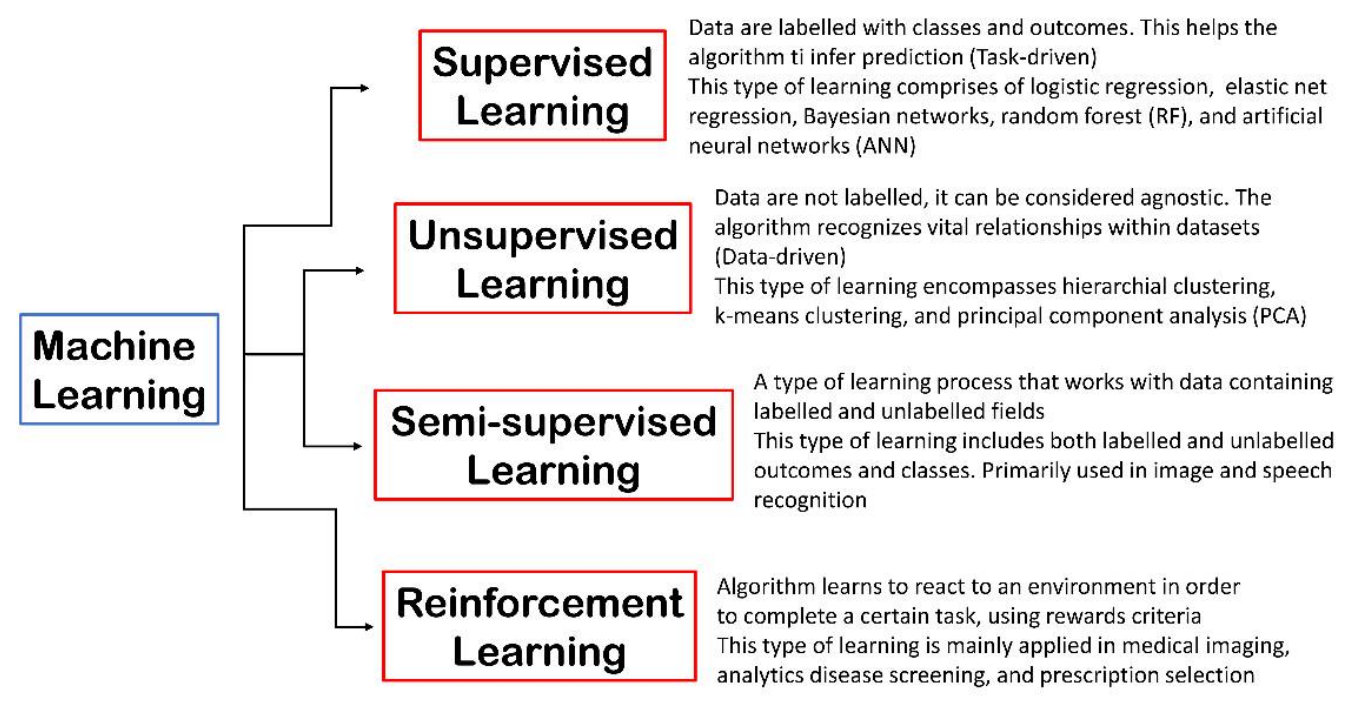

Figure 3. ML is mainly classified into four classes: supervised learning, semi-supervised learning, unsupervised learning, and reinforcement learning.

However, it is important to highlight that the accuracy of these analyses is strongly dependent on the quality of the training sets employed to generate ML models.

Finally, the reinforcement ML method allows the computational tool to learn from its failures, generating an algorithm based on what it has learned. Thus, this learning is constructed upon the trial-and-error process [26]. In the scientific field, for example, different tasks can include training an algorithm to understand the treatment regimens of medical registry data and to find the optimal strategy for treating patients with chemotherapy. A recent study reported the successful use of a reinforcement ML model for establishing an effective formulation of clinical trial dosing. The algorithm was trained with proper dosing regimens for reducing tumor diameters in patients treated by means of chemotherapy and radiation [27]. In Figure 3 is reported a schematic illustration of the mentioned ML approaches.

This brief excursion about the different ML techniques and how they can be applied to scientific fields, for improving and enhancing the understanding of complex systems, highlights the potential of ML methods. To this end, there is growing attention being paid to the application of these methodologies in the framework of translational medicine, enhancing the ability of translational scientists to provide novel effective treatments and diagnostics for healthcare. In this paper, we analyze the most advanced AI/ML methods applied to translational medicine that can learn from a range of big data sets produced in the lab and be utilized for accomplishing jobs that are difficult for human scientists. In particular, we report $\mathrm{AI} / \mathrm{ML}$, the most relevant and innovative approaches in different 
areas of medicine, with a particular focus on (a) drug discovery and development and (b) imaging, biomarkers, diagnosis, and disease progression.

\section{Artificial Intelligence (AI) and Machine Learning (ML) in Translational Medicine \\ 2.1. Drug Discovery and Development, and Drug Target Prediction \\ 2.1.1. Drug Discovery and Development}

Beyond the classical computational approaches in drug discovery, such as ligand(mainly QSAR methods and pharmacophore modeling) [28-34] and structure-based strategies (mainly based on molecular docking and molecular dynamics) [35-43] or a combination of them [44-49], currently these computational methods are integrated with ML technologies for improving the reliability of the calculation, avoiding false positive outcomes and enhancing the success ratio in identifying safer hit compounds. Some examples are represented by QSAR-ML models [50-53], and multi- and combi-QSAR approaches [54-60]. Furthermore, in the drug discovery field, advanced computational models, based on ML technology, have demonstrated strong potential in selecting effective hit compounds [61-68]. Moreover, ML-based approaches represent a valuable resource also in the drug repurposing field $[69,70]$. Interestingly, these approaches have provided potential drugs for treating COVID-19 in a short time [71,72]. Currently, protein structural modeling and design, as well as protein structure prediction, which can increase the proficiency in the drug discovery pipeline, are emerging areas of application of ML models [73-76]. In fact, ML methods offer a theoretical framework for identifying and prioritizing bioactive molecules possessing suitable pharmacological profile, as well as optimizing them as drug-like lead compounds before clinical investigation [68]. Generally, three steps allow the development of a computational protocol enabling ML-based models: (a) the selection of appropriate descriptors for capturing crucial features of compounds involved in the study; (b) the selection of a suitable metric or scoring system for comparing the set of molecules; (c) a proper ML-based technique for determining the characteristic traits of the features that help to qualitatively or quantitatively discriminate active molecules from inactive ones [77,78]. ML/DL approaches suitable in the drug discovery field include RF, Artificial Neural Networks (ANN), Deep Neural Networks (DNN), Graph Convolutional Neural Networks (GCNN), Convolutional Neural Networks (CNN), Naïve Bayesian techniques, Multiple Linear Regression (MLR), natural language processing (NLP), decision trees (DT), Logistic Regression (LR), Linear Discriminant Analysis (LDA), Multi-Layer Perceptron (MLP), Probabilistic Neural Networks (PNN), k-nearest neighbors (k-NN), and Support Vector Machine (SVM), only considering some of them in the context of ML [77,79,80].

Briefly, we report some successful and representative examples in which ML-based methods enable the discovery of interesting hit compounds against different targets (Table 1). Vignaux and colleagues used publicly available data in ChEMBL (https: / /www.ebi.ac.uk/ chembl/; accessed on 14 October 2021) [81] for building and validating Bayesian ML models for Alzheimer's disease (AD) drug targets. The first selected target was glycogen synthase kinase 3 beta (GSK-3 $\beta$ ), a well-established protein for the design of anti-AD drugs. GSK$3 \beta$ is a proline-directed serine-threonine kinase able to phosphorylate the microtubulestabilizing tau protein. The process causes dissociation of the microtubule, forming insoluble oligomers that are the constituents of neurofibrillary tangles detected in AD brains. The authors developed and validated a Bayesian ML (supervised ML) model for GSK-3 $\beta$ considering 2368 compounds (cross validation, receiver operating characteristic (ROC) curve $=0.905$ ). Hence, the developed computational tool was used for virtually screening a chemical library containing FDA-approved and investigational drugs. Experimental validation showed that following this protocol, the authors selected a series of structurally different GSK-3 $\beta$ inhibitors. Among the retrieved active compounds, a selective small-molecule inhibitor (ruboxistaurin, CHEMBL91829), showing activity against GSK-3 $\beta$ $\left(\mathrm{IC}_{50}=97.3 \mathrm{nM}\right)$ and GSK-3 $\alpha\left(\mathrm{IC}_{50}=695.9 \mathrm{nM}\right)$, deserves particular attention. This interesting approach highlights the valuable help of ML for accelerating the drug discovery process for finding effective AD therapeutic agents [63]. Fang and coworkers combined Bayesian 
ML and recursive partitioning (RP) algorithms for building classifiers to predict the activity of molecules on 25 crucial cellular targets in $\mathrm{AD}(18,741$ active compounds for the selected targets from bindingDB database, https:/ / www.bindingdb.org/bind/index.jsp; accessed on 14 October 2021) applying a multitarget quantitative structure-activity relationships (multi-QSAR) approach. The authors started to describe the selected molecules with two types of fingerprint descriptors, namely, ECFP6 and MACCS; afterwards, they built one hundred classifiers. The performance was assessed by internal and external validation (area under the ROC curve for the test sets 0.741-1.0, average 0.965). The obtained values are indicative of robust models. The validated computational tools were used for predicting the possible targets for six approved anti-AD drugs and 19 known active molecules within the AD framework. The experimental validation confirmed the prediction outcomes, with the identification of various multitarget-directed ligands (MTDLs) against $\mathrm{AD}$ (seven acetylcholinesterase $(\mathrm{AChE})$ inhibitors $\left(\mathrm{IC}_{50}=0.442-72.26 \mu \mathrm{M}\right)$; four histamine receptor $3\left(\mathrm{H}_{3} \mathrm{R}\right)$ antagonists $\left(\mathrm{IC}_{50}=0.308-58.6 \mu \mathrm{M}\right)$ ). Among the retrieved active compounds, the best MTDL, namely, DL0410, showed a dual cholinesterase inhibitor behavior $\left(\mathrm{IC}_{50} \mathrm{AChE}=0.442 \mu \mathrm{M} ; \mathrm{IC}_{50}\right.$ butyrylcholinesterase $\left.(\mathrm{BuChE})=3.57 \mu \mathrm{M}\right)$. Moreover, DL0410 behaved as a $\mathrm{H}_{3} \mathrm{R}$ antagonist, showing an $\mathrm{IC}_{50}$ of $0.308 \mu \mathrm{M}$. Remarkably, the selected work could have implications in MTDL research against other disorders [82]. Remaining in the AD context, Rodriguez and colleagues reported the development of DRIAD (Drug Repurposing In AD), an ML-based strategy for quantifying possible relationships between the pathology of AD severity (the Braak stage) and molecular mechanisms as determined in records of gene names. Authors applied DRIAD to lists of genes arising from perturbations in differentiated human neural cells by using 80 FDA-approved and investigational drugs, identifying potential drugs for repurposing. The top-ranked drugs were experimentally evaluated against their targets. Interestingly, the results show that 33 FDA-approved drugs can be used for repurposing immediately. Notably, these selected drugs, after the supplementary validation and identification of significant pharmacodynamic biomarkers, could be directly investigated in human clinical trials [69]. Considering another neurodegenerative disorder, such as Parkinson's disease (PD), Shao and collaborators described an integrated computational platform based on two in silico methods. The ML approach was represented by SVM models coupled with Tanimoto similarity-based clustering analysis (135 compounds in total, 96 from $\mathrm{A}_{2 \mathrm{~A}}$ and 39 from $\mathrm{D}_{2}$ ). Following this strategy, the authors investigated the possibility to identify molecules possessing an indole-piperazine-pyrimidine scaffold, able to modulate human adenosine receptor $\mathrm{A}_{2 \mathrm{~A}}$ and human dopamine receptor $\mathrm{D}_{2}$ subtypes. They identified two compounds that behaved as multifunctional ligands against human $\mathrm{A}_{2 \mathrm{~A}}\left(K_{\mathrm{i}}=8.7\right.$ and $\left.11.2 \mu \mathrm{M}\right)$ and $\mathrm{D}_{2}$ receptors $\left(\mathrm{EC}_{50}=22.5\right.$ and $\left.40.2 \mu \mathrm{M}\right)$. Furthermore, the retrieved hit compounds did not show any mutagenicity (up to $100 \mu \mathrm{M}$ ), cardiotoxicity, or hepatotoxicity (up to $30 \mu \mathrm{M}$ ) issues, and one molecule improved the movement and mitigation concerning the loss of dopaminergic neurons in Drosophila models of PD [83]. In the same field, Michielan and coworkers reported a different application of the SVM and Support Vector Regression (SVR) methods for describing $\mathrm{A}_{2 \mathrm{~A}}$ versus $\mathrm{A}_{3}$ receptor subtype selectivity profiles, as well as related binding affinities. The authors implemented an integrated application of the SVM-SVR method, constructed on the usage of molecular descriptors encoding for the molecular electrostatic potential (autoMEP). In this way, the computational tool can simultaneously distinguish $\mathrm{A}_{2 \mathrm{~A}}$ versus $\mathrm{A}_{3}$ receptor antagonists, predicting their binding affinity to the corresponding receptor subtype of a huge dataset composed of pyrazolo-triazolo-pyrimidine derivatives. The in silico approach was experimentally validated by synthesizing 51 novel pyrazolo-triazolo-pyrimidine-containing compounds, which confirmed the predicted receptor subtype selectivity and related binding affinity profiles [84].

Regarding the anticancer research, Deshmukh and colleagues employed two ML algorithms (SVM and RF) for generating four classification models, considering a large amount of PubChem bioassay data and probable human Flap endonuclease1 (FEN1) inhibitors and non-inhibitors (the training set contained 1163 FEN1 inhibitors and 281,583 non-inhibitors; 
the test set 388 inhibitors and 93,861 non-inhibitors). FEN1 is a crucial protein concerning DNA replication and repair processes. Accordingly, the inhibition of Flap cleavage action results in increased cellular sensitivity to DNA-damaging agents (e.g., cisplatin, temozolomide), with the possibility of improving cancer prognosis. Since FEN1 is overexpressed in several kinds of tumors, FEN1 inhibitors could represent efficacious anticancer agents. For developing the mentioned ML models, the authors used huge, freely accessible, high-throughput screening data regarding small molecules targeting FEN1. The findings showed that the SVM model with inactive molecules was superior to RF, with a Matthews's correlation coefficient (MCC) of 0.67 for the test set. The computational tool was subsequently used in a virtual screening employing the Maybridge database (53,000 molecules). Five top-ranked compounds were experimentally validated. In fact, the selected hit compounds were tested against the enzyme and in the cell-based system. The molecule JFD00950 behaved as a FEN1 inhibitor in the micromolar range, inhibiting Flap cleavage activity, Moreover, JFD00950 showed cytotoxic activity against colon cancer cells (DLD-1, $\mathrm{IC}_{50}=16.7 \mu \mathrm{M}$ ) [85]. The exploration of another drug target for developing anticancer drugs was performed by Zhang and colleagues. They investigated a promising target for cancer immunotherapy, the indoleamine 2,3-dioxygenase (IDO). The authors generated ML models using naïve Bayesian and RP techniques, considering a library of established IDO inhibitors (504 compounds, 242 active compounds and 262 inactive compounds). For building the models, they used descriptors employing 13 molecular fingerprints for predicting IDO inhibitors. Model performances were evaluated in silico, showing that the $\mathrm{Q}$ values of the top 10 models are greater than 0.76 , the MCC values are greater than 0.53 , and the values of the area under the ROC curve are greater than 0.89 . The best-performing ML computational tool was employed in a virtual screening campaign using a proprietary chemical library. This step provided 50 potential IDO inhibitors that were experimentally validated. In vitro tests confirmed the prediction provided by the ML model, since three new IDO inhibitors, belonging to the tanshinone family, were identified $\left(\mathrm{IC}_{50}=1.30,4.10\right.$, and $\left.4.68 \mu \mathrm{M}\right)$ [86]. Kang and coworkers attempted to target vascular endothelial growth factor receptor 2 (VEGFR-2), a well-established target for developing anticancer compounds with anti-angiogenic activity. The authors developed an ML model using the naïve Bayesian technique coupled with a molecular docking calculation, obtaining a virtual screening protocol that was used to identify VEGFR-2 inhibitors using a chemical library containing FDA-approved drugs. The most promising naïve Bayesian model, developed considering 3464 VEGFR-2 inhibitors, showed an MCC of 0.966 and 0.951 considering the test set and external validation set, respectively. Accordingly, using the developed computational model, 1841 FDA-approved drugs were screened and subsequently submitted to a molecular docking calculation employing LibDock. The outcome of virtual screening provided nine top-ranked drugs showing an EstPGood value $\geq 0.6$ and LibDock Score $\geq 120$, which were submitted for biological evaluation. VEGFR-2 kinase test results show that papaverine, rilpivirine, and flubendazole were able to inhibit VEGFR-2 $\left(\mathrm{IC}_{50 \mathrm{~s}}=0.47-6.29 \mu \mathrm{M}\right)$. Notably, the integrated screening platform provided three FDAapproved drugs as new VEGFR-2 inhibitors, that can be rapidly translated into clinical studies [87]. Montanari and coworkers applied four distinct ML algorithms to train the model (LR, naïve Bayesian, SVM, and RF) for identifying novel agents acting as breast cancer resistance protein (BCRP) inhibitors. BCRP is involved in multidrug resistance (MDR) events, thus emerging BCRP inhibitors for increasing the concentration of antitumor agents into resistant cancer cells has been proposed as a valuable tactic for overcoming MDR. The developed model, using 433 inhibitors and 545 noninhibitors, was validated, showing good predictivity in cross-validation (area under ROC curve $=0.9$ ) and satisfactory predictivity in prospective validation (area under ROC curve $=0.7$ ). Subsequently, the computational tool was employed in a virtual screening approach using the drug library (1702 compounds). Following this strategy, the authors identified 10 drugs as potential BCRP inhibitors to submit for biological evaluation (inhibition of mitoxantrone efflux in BCRP-expressing PLB985 cells). Among the drugs tested, two of them behaved as BCRP inhibitors (cisapride 
and roflumilast, $\mathrm{IC}_{50}=0.4 \mu \mathrm{M}$ and $0.9 \mu \mathrm{M}$, respectively) [88]. Allen and collaborators used an ML model, based on Laplacien-modified naïve Bayesian classifiers developed considering topological fingerprints, in a virtual screening campaign employing a large database (eMolecules $>6$ million compounds) for selecting dual kinase/bromodomain (EGFR/BRD4) inhibitors. Two ML models for EGFR were developed considering extended connectivity fingerprints (ECFP4) based on a total of 591,744 unique kinase compounds: one with 3058 active molecules characterized by a $\mathrm{pIC}_{50} / \mathrm{pK}_{i} \geq 7$, and another with 4785 active compounds with $\mathrm{pIC}_{50} / \mathrm{pK}_{i} \geq 6$. The two developed models showed exceptional area under the ROC curve values of 0.98 to 0.99 based on a 50/50 training/test set and assessed by employing leave-one-out cross-validations. The enrichment factors considering $1 \%$ of the dataset were 78 and 66, respectively. The ML model for kinase was coupled with a structure-based technique regarding the bromodomain. This computational protocol allowed the identification of various BRD4 inhibitors. Among them, a first-in-class dual EGFR-BRD4 inhibitor (compound 2870) was found (EGFR IC $50=44 \mathrm{nM}$; ERBB2, ERBB4, and BRD4 $\mathrm{IC}_{50}=8.73,24.2$, and $9.02 \mu \mathrm{M}$, respectively) [89].

In the field of parasitic and neglected tropical diseases, ML-based approaches can be useful for identifying novel effective therapeutic agents, as recently reported [90]. Here, we only highlighted the representative works explicative of the mentioned technology. Keshavarzi Arshadi and colleagues developed an ML model based on a GCNN algorithm. GCNN has demonstrated strong accuracy for predictions concerning the chemical properties of molecules. These ML-based computational models transform the molecules into graphs and learn higher-level abstract representations of the input solely based on the data [91]. In the above-mentioned research, GCNN represent the core of a new AI platform called DeepMalaria, with the aim to speed up the antimalarial drug discovery. The characteristic capacities of GCNNs are employed for implementing a virtual screening pipeline. A graph-based model was trained on 13,446 potential antimalarials contained in the GlaxoSmithKline database. The developed model was validated by predicting hit molecules from an additional chemical collection and an FDA-approved drug database. The molecules were also tested by employing in vitro tests for validating the ML-based model. DeepMalaria identified all molecules, showing nanomolar activity and $87.5 \%$ of the chemicals having a greater percentage of inhibition ( $>50 \%)$. Additional tests to uncover the mechanism of action of compounds showed that one of the hit molecules, DC-9237, not only inhibits all asexual stages of Plasmodium falciparum, but is a fast-acting molecule, making it a robust drug candidate to be optimized [92]. Furthermore, a very interesting ML-based approach was reported by Stokes and collaborators regarding the application of the DL method for the discovery of novel antibiotic agents. Due to the tremendous impact of antibiotic resistance in clinical practice, there is an urgent need for novel chemicals able to inhibit multidrug resistance bacteria [93]. In the mentioned work, the scientists trained a DNN model, using a dataset of 2335 molecules, for identifying compounds possessing a broad-spectrum antibacterial profile. The obtained computational tool exhibited an area under the ROC curve of 0.896 considering the test data. As a result, the authors employed the model for screening various chemical libraries. From this screening step, they identified an existing drug, namely, halicin (SU-3327, developed for inhibiting c-June N-terminal kinase (JNK)). Remarkably, the structure of this compound is totally different from classical antibiotic agents. Moreover, halicin was demonstrated to possess interesting bactericidal activity in vitro as well as in vivo. The characterization of the mechanism of action as an antibiotic revealed that halicin can dissipate the transmembrane $\Delta \mathrm{pH}$ potential in bacteria, and it was found to be effective against $M$. tuberculosis. Moreover, the developed ML model was used to screen over 100 million compounds belonging to the ZINC15 database. This additional screening provided eight further antibacterial agents, chemically unrelated to well-known antibiotics. Among them, two compounds (ZINC000100032716 and ZINC000225434673) showed strong broad-spectrum activity and overcame a range of frequent resistance factors. This approach was the first effective experiment regarding the application of DNN for drug repurposing and for discovering new drug lead compounds. The findings indicate 
that ML approaches can be relevant for identifying novel antibiotic agents, counteracting the dissemination of resistance, decreasing the assets required for discovering these compounds, and reducing the associated costs [94]. In another investigation, Li and colleagues generated ML models, employing naïve Bayesian and RP techniques, based on physicochemical descriptors and structural fingerprints (137 DNA gyrase inhibitors with an $\mathrm{IC}_{50}$ ranging from nanomolar to high micromolar), aimed at identifying novel DNA gyrase inhibitors to develop broad-spectrum antibacterial agents, bacterial DNA gyrase not being expressed in eukaryotic cells. The overall predictive accuracy, considering the training and test sets, was greater than $80 \%$. The authors used eleven promising ML models for the virtual screening of a chemical library. The potential hits, selected by virtual screening, were experimentally validated against Escherichia coli, methicillin-resistant Staphylococcus aureus and other bacteria, and DNA gyrase. For compounds able to inhibit DNA gyrase, MIC values range between 1 and $32 \mu \mathrm{g} / \mathrm{mL}$, and the relative inhibition rates of inhibitors range from $42 \%$ to $75 \%$ at $1 \mu \mathrm{M}$ [95].

In the context of antiviral research, Ekins and collaborators developed a Bayesian ML model considering viral pseudotype entry assay and the Ebola virus replication assay data (868 molecules). The developed model was submitted to an internal and external validation step (area under ROC curve greater than 0.8). The scientists employed this model in a virtual screening campaign using the MicroSource library of drugs, for selecting possible antiviral compounds. Among the retrieved potential hit compounds, three promising antiviral candidates were found (quinacrine, pyronaridine, and tilorone were experimentally validated with an $\mathrm{EC}_{50}=350,420$, and $230 \mathrm{nM}$, respectively, against Ebola virus replication). Notably, pyronaridine is an element of a combination therapy for malaria recently approved by the European Medicines Agency (EMA); consequently, it could be immediately used for clinical testing. Additionally, this study highlighted how ML models can be used for speeding up the preclinical step of the drug discovery trajectory, providing drugs for translational research [96].

Remarkably, ML approaches, especially based on reinforcement learning, can be useful for developing models that can also be applied for the de novo design of small molecules possessing desired pharmacological profiles [97-99]. Briefly, we report some representative attempts to apply this methodology to this task. Recently, Zhavoronkov and coworkers reported the development of a deep generative model, namely, generative tensorial reinforcement learning (GENTRL), useful for de novo small molecule design, acting as inhibitors of discoidin domain receptor 1 (DDR1) kinase, which is involved in fibrosis and further disorders. To develop GENTRL, the authors combined reinforcement learning, variational inference, and tensor decompositions into a generative two-step ML algorithm. In the first step, the scientists mapped the chemical space, a set of discrete molecular graphs, to a continuous space of 50 dimensions, parameterizing the structure of the learned manifold in the tensor train format to utilize partly well-known features. The computational model was generated using six data sets: (i) a big set of compounds from ZINC database; (ii) known inhibitors of DDR1 kinase; (iii) common kinase inhibitors (positive set); (iv) compounds active against non-kinase target proteins (negative set); (v) patent data of pharmaceutical companies regarding biologically active compounds; and (vi) 3D structures for DDR1 inhibitors. In the second step, they explored the mapped chemical space with reinforcement learning for the discovery of novel molecules against a selected target. The results show that GENTRL is capable of optimizing synthetic accessibility, novelty, and bioactivity. In the reported paper, GENTRL allowed the indication of several compounds for the synthesis, and the authors synthesized six lead compounds. These latter were experimentally evaluated for their inhibitory potential against DDR1. Notably, two molecules strongly inhibited DDR1 activity $\left(\mathrm{IC}_{50}=10-21 \mathrm{nM}\right)$, the other two compounds showed moderate potency $\left(\mathrm{IC}_{50}=0.278-1 \mu \mathrm{M}\right)$, while the remaining two molecules were found to be inactive. Moreover, the best-performing compounds demonstrated good selectivity against DDR1 over DDR2, and one was highly selective against a panel of 44 diverse kinases. Interestingly, these two compounds inhibited the induction of fibrotic markers $(\alpha-$ 
actin and CCN2) in MRC-5 lung fibroblasts. These chemical entities were able to inhibit the expression of collagen (a hallmark of fibrosis) in LX-2 hepatic stellate cells [100]. McCloskey and coworkers, in an interesting approach, described an effective ML platform aimed at accelerating the drug discovery pipeline considering DNA-encoded small molecule library (DEL) selection data. Two types of ML models were trained on the DEL selection data for classifying molecules (over 2000): RF and GCNN. ML models were trained on the aggregated selection data (using no prior off-DNA activity measurements). The computational tool was applied to three drug targets ( $\mathrm{SEH}$ (a hydrolase), ER $\alpha$ (a nuclear receptor), and c-KIT (a kinase)) and used in the virtual screening of large chemical databases ( 88 million compounds). The outcomes revealed that the technique is efficient, with a global hit rate of $\sim 30 \%$ at $30 \mu \mathrm{M}$, discovering powerful compounds ( $\mathrm{IC}_{50}<10 \mathrm{nM}$ ) for each drug target [101]. Lastly, a novel ML approach based on DL and reinforcement learning for the de novo design of small molecules with desirable profiles was presented by Popova and coworkers. This computational tool, named ReLeaSE (Reinforcement Learning for Structural Evolution), combines two DNNs (generative and predictive) that are trained independently, although are employed together for generating new focused chemical libraries. The methodology was separated into two phases, in the first one, a supervised learning algorithm was employed for a separate training of generative and predictive models. The second phase consisted of a joint training of both models with the reinforcement learning methodology to bias the generation of new chemicals showing the desired physical and biological profile. In the work, the authors applied ReLeaSE for generating a series of libraries containing chemical entities with a precise profile: (a) satisfactory drug-likeness, regarding physchem properties, for which the authors chose $T_{\mathrm{m}}$ and $n$-octanol/water partition coefficient $(\log P)$; (b) desired biological activity, for which the authors selected Janus protein kinase 2 (JAK2) as the target protein; and (c) novel chemotypes with significant chemical complexity, that should guarantee a higher selectivity against the selected target. In particular, the number of benzene rings and substituents was employed as a structural reward for designing focused libraries enclosing chemically complex molecules [97].

\subsubsection{Drug Target Prediction and Biomarker Identification}

Noteworthy is that in addition to the previously discussed ML approaches to identify promising drug candidates, AI techniques are also emerging in drug target prediction, with remarkable success. For instance, in the field of neurodegenerative disorders, we report here significant progress in ML approaches applied to drug target identification in the drug discovery/drug repurposing field (Table 2). In fact, a computational model based on DL methodology, namely, deepDTnet was successfully used in a repurposing approach, providing interesting hints for treating multiple sclerosis [102]. DeepDTnet was conceived for identifying novel drug targets and for drug repurposing, considering the heterogeneous drug-gene-disease network, embedding fifteen categories of chemical, genomic, phenotypic, and cellular network profiles. DeepDTnet was generated using 732 FDA-approved drugs for training. Subsequent validation analysis showed that deepDTnet was accurate in identifying innovative cellular drug targets for marketed drugs (area under the ROC curve $=0.963$ ). The experimental validation was performed considering the output of topotecan (a topoisomerase-I inhibitor), a chemotherapeutic agent approved to treat various forms of cancer, such as lung and ovarian cancer [103-105]. In fact, topotecan was predicted by deepDTnet as an inhibitor of the human retinoic-acid-receptor-related orphan receptor-gamma $\mathrm{t}(\mathrm{ROR}-\gamma \mathrm{t})$, a promising drug target for treating different disorders including psoriasis, multiple sclerosis, and rheumatoid arthritis [106,107]. According to the computational output, topotecan was found to inhibit ROR- $\gamma \mathrm{t}\left(\mathrm{IC}_{50}=0.43 \mu \mathrm{M}\right)$ and notably showed potential therapeutic effects in multiple sclerosis, being effective in reverting the pathological phenotype in vivo in the EAE mouse model at $10 \mathrm{mg} / \mathrm{kg}$ [102]. Madhukar and colleagues, in the framework of drug target identification, developed a Bayesian ML algorithm, namely, BANDIT (Bayesian ANalysis to determine Drug Interaction Targets). This computer-based tool combines various kinds of data for predicting drug targets (e.g., 
20 million data points derived from six diverse types of data, such as drug efficacy, posttreatment transcriptional response, drug structure, described undesirable effects, bioassay results, and well-established targets). Using over 2000 compounds, BANDIT showed an accuracy of $\sim 90 \%$ in identifying correct targets. Next, the authors used this computational platform employing over 14,000 molecules for which any target was known. The results show that the ML-based tool produced $\sim 4000$ undisclosed molecule target predictions. Considering the most promising data, the authors validated fourteen molecules predicted as microtubule binders. Among this subset, three compounds were highlighted for their activity against resistant tumor cells. Experimental validation fully supported the BANDIT predictions. Moreover, BANDIT was applied to ONC201 (anticancer agents in clinical development with an unknown target). The development algorithm predicted ONC201 as an antagonist of the $\mathrm{D}_{2}$ receptor. The target was validated confirming the prediction, and currently this hint derived from the mentioned studies was the basis for designing an appropriate clinical trial using ONC201. ONC201 will be evaluated for its efficacy in pheochromocytomas, a rare cancer in which was observed an overexpression of $\mathrm{D}_{2}$ receptor (NCT03034200). Lastly, BANDIT identified linkers among distinct classes of drugs, revealing undisclosed clinical observations, highlighting novel possibilities for drug repurposing. According to these findings, BANDIT is a useful screening platform that can efficiently speed up the drug discovery process, accelerating translational research toward clinical application [108]. Dezső and Ceccarelli reported the development of an ML-based approach for scoring proteins for generating a druggability score of novel unidentified drug targets. The authors included in the ML model 70 features obtained from drug targets (e.g., features indicating protein functions, features extracted from the sequence, and network features obtained from the protein-protein interaction network). They generated 10,000 ML models based on the RF algorithm using a training set built considering drug targets in complex with marketed drugs (102 targets), and a "negative" set enclosing 102 non-drug targets. The developed ML models were able to detect relevant combinations of included features, discriminating drug targets from non-pharmacological targets. The approach was validated using an external test set of clinically relevant drug targets (277 targets). The validation results showed a significant accuracy, accounting for an area under the ROC curve of 0.89 . The authors further validated their predictions using an independent set of clinical drug targets, attaining a high accuracy, as indicated by an area under the ROC curve of 0.89 . The output reported in this work provided new potential drug targets for developing innovative anticancer drugs [109]. 
Table 1. Main examples of AI/ML in the drug discovery and development field.

\begin{tabular}{|c|c|c|c|c|c|}
\hline AI Technique & Target & Dataset & $\begin{array}{c}\text { Statistical } \\
\text { Parameters }\end{array}$ & Outcomes & Ref \\
\hline $\begin{array}{l}\text { Bayesian ML and RP algorithms for } \\
\text { developing a multi-QSAR approach }\end{array}$ & 25 crucial cellular targets in $\mathrm{AD}$ & $\begin{array}{l}\text { 18,741 active compounds against } \\
\text { the selected targets }\end{array}$ & $\begin{array}{l}\text { Cross-validation, ROC curve }=0.905 \\
\text { Internal and external validation (area } \\
\text { under the ROC curve for the test set } \\
\quad 0.741-1.0 \text {, average } 0.965 \text { ) }\end{array}$ & $\begin{array}{c}\text { Virtual screening found ruboxistaurin } \\
\text { (CHEMBL91829) as GSK-3 } 3\left(\mathrm{IC}_{50}=97.3 \mathrm{nM}\right) \text { and } \\
\text { GSK-3 } \alpha\left(\mathrm{IC}_{50}=695.9 \mathrm{nM}\right) \text { inhibitor } \\
\text { Identification of various MTDLs against } \mathrm{AD} \\
\text { (seven AChE inhibitors }\left(\mathrm{IC}_{50}=0.442-72.26 \mu \mathrm{M}\right) \\
\text { four } \mathrm{H}_{3} \mathrm{R} \text { antagonists }\left(\mathrm{IC} \mathrm{C}_{50}=0.308-58.6 \mu \mathrm{M}\right) \text {. The } \\
\text { best performing MTDL }(\mathrm{DL} 0410) \text { showed a dual } \\
\text { cholinesterase inhibitor behavior } \\
\left(\mathrm{IC}_{50} \mathrm{AChE}=0.442 \mu \mathrm{M} ; \mathrm{IC}_{50} \text { BuChE }=3.57 \mu \mathrm{M}\right) \\
\text { and behaved as a } \mathrm{H}_{3} \mathrm{R} \text { antagonist } \\
\left(\mathrm{IC}_{50}=0.308 \mu \mathrm{M}\right)\end{array}$ & [63] \\
\hline ML-based approach & $\begin{array}{l}\text { DRIAD for drug repurposing } \\
\text { in AD }\end{array}$ & $\begin{array}{l}\text { DRIAD was applied to find } \\
\text { relationships between the } \\
\text { pathology of AD severity (the } \\
\text { Braak stage) and molecular } \\
\text { mechanisms as determined in } \\
\text { records of gene names by using } \\
80 \text { FDA-approved and } \\
\text { investigational drugs }\end{array}$ & $\begin{array}{l}\text { Model performance was evaluated } \\
\text { through leave-pair-out } \\
\text { cross-validation, area under the ROC } \\
\text { curve ranging from } 0.6 \text { to } 0.8\end{array}$ & $\begin{array}{l}33 \text { FDA-approved drugs can be used for } \\
\text { repurposing immediately }\end{array}$ & [69] \\
\hline $\begin{array}{l}\text { SVM models coupled with Tanimoto } \\
\text { similarity-based clustering analysis }\end{array}$ & $\begin{array}{c}\mathrm{A}_{2 \mathrm{~A}} \text { and } \mathrm{D}_{2} \text { receptor subtypes as } \\
\text { targets for } \mathrm{PD}\end{array}$ & $\begin{array}{l}135 \text { compounds ( } 96 \text { from } A_{2 A} \\
\text { and } 39 \text { from } D_{2} \text { ) }\end{array}$ & Experimental validation & $\begin{array}{l}\text { Virtual screening of over } 13.5 \text { million compounds } \\
\text { from PubChem and MDDR databases. } \\
\text { Two compounds behaved as multifunctional } \\
\text { ligands against human } \mathrm{A}_{2 \mathrm{~A}}(\mathrm{Ki}=8.7 \text { and } 11.2 \mu \mathrm{M}) \\
\text { and } \mathrm{D}_{2} \text { receptors }\left(\mathrm{EC}_{50}=22.5 \text { and } 40.2 \mu \mathrm{M}\right)\end{array}$ & [83] \\
\hline SVM and SVR & $\begin{array}{l}\text { PD drug discovery } \mathrm{A}_{2 \mathrm{~A}} \text { vs. } \mathrm{A}_{3} \\
\text { receptor subtype selectivity } \\
\text { profiles and related } \\
\text { binding affinities }\end{array}$ & $\begin{array}{l}\text { For SVM, } 104 \text { selective } \mathrm{N}^{7} \text { - and } \\
\mathrm{N}^{8} \text {-substituted } \\
\text { pyrazolo-triazolo-pyrimidine } \\
\text { analogs. For SVR, } 104 \\
\mathrm{~N}^{8} \text {-substituted } \\
\text { pyrazolo-triazolo-pyrimidine } \\
\text { derivatives. } \\
\text { A test set of } 51 \mathrm{~N}^{8} \text {-substituted } \\
\text { pyrazolo-triazolo-pyrimidine } \\
\text { analogs to validate both SVM } \\
\text { and SVR models }\end{array}$ & $\begin{array}{c}\text { LOO-cv } \\
\text { Correct prediction 93.3, } \\
\text { sensitivity 92.0, specificity } 94.4\end{array}$ & $\begin{array}{l}51 \text { novel pyrazolo-triazolo-pyrimidine containing } \\
\text { compounds that confirmed the predicted receptor } \\
\text { subtype selectivity and the related binding } \\
\text { affinity profiles }\end{array}$ & [84] \\
\hline
\end{tabular}


Table 1. Cont.

\begin{tabular}{|c|c|c|c|c|c|}
\hline AI Technique & Target & Dataset & $\begin{array}{l}\text { Statistical } \\
\text { Parameters }\end{array}$ & Outcomes & Ref \\
\hline SVM and RF & $\begin{array}{c}\text { Anticancer drug } \\
\text { discovery—target FEN1 }\end{array}$ & $\begin{array}{l}\text { The training set contained } \\
1163 \text { FEN1 inhibitors and } \\
\text { 281,583 non-inhibitors; the test } \\
\text { set } 388 \text { inhibitors and } \\
93,861 \text { non-inhibitors }\end{array}$ & $\begin{array}{c}\text { For the test set: } \\
\text { sensitivity } 0.54 \text {, specificity } 0.99 \text {, } \\
\text { MCC } 0.67\end{array}$ & $\begin{array}{l}\text { The computational tool was used in a virtual } \\
\text { screening employing the Maybridge database } \\
\text { (53,000 molecules). Five top-ranked compounds } \\
\text { were experimentally validated. The molecule } \\
\text { JFD00950 behaved as a FEN1 inhibitor in the } \\
\text { micromolar range, inhibiting Flap cleavage } \\
\text { activity, showing cytotoxic activity against colon } \\
\text { cancer cells (DLD-1, IC } 50=16.7 \mu \mathrm{M} \text { ) } \\
\text { Virtual screening campaion using a proprietary }\end{array}$ & [85] \\
\hline $\begin{array}{l}\text { ML models using naïve Bayesian and } \\
\text { RP techniques }\end{array}$ & $\begin{array}{l}\text { Indoleamine 2,3-dioxygenase } \\
\text { (IDO), a promising target for } \\
\text { cancer immunotherapy }\end{array}$ & $\begin{array}{l}\text { The model was trained using a } \\
\text { library of established IDO } \\
\text { inhibitors (504 compounds, } \\
242 \text { active and } 262 \text { inactive) }\end{array}$ & $\begin{array}{l}\text { The } Q \text { values for the test set of the top } \\
10 \text { models are greater than } 0.76 \text {, the } \\
\text { MCC values }>0.53 \text {, the area under } \\
\text { ROC curve }>0.89\end{array}$ & $\begin{array}{l}\text { Virtual screening campaign using a proprietary } \\
\text { chemical library. This step provided } 50 \text { potential } \\
\text { IDO inhibitors that were experimentally validated. } \\
\text { In vitro tests confirmed the prediction of the ML } \\
\text { model, since three new IDO inhibitors, belonging } \\
\text { to the tanshinone family, were identified } \\
\left(\mathrm{IC}_{50} \mathrm{~s}=1.30,4.10 \text {, and } 4.68 \mu \mathrm{M}\right)\end{array}$ & [86] \\
\hline $\begin{array}{l}\text { ML model using naïve Bayesian } \\
\text { technique coupled with a molecular } \\
\text { docking calculation }\end{array}$ & $\begin{array}{l}\text { VEGFR-2, a drug target for } \\
\text { developing anticancer } \\
\text { compounds with } \\
\text { anti-angiogenic activity }\end{array}$ & $\begin{array}{l}\text { The model was trained using } \\
3464 \text { VEGFR-2 inhibitors }\end{array}$ & $\begin{array}{l}\text { MCC of } 0.966 \text { and } 0.951 \text { considering } \\
\text { the test set and external validation set }\end{array}$ & $\begin{array}{l}\text { Virtual screening protocol for identifying } \\
\text { VEGFR-2 inhibitors using a chemical library } \\
\text { containing } 1841 \text { FDA-approved drugs. } \\
\text { Papaverine, rilpivirine, and flubendazole were } \\
\left.\text { able to inhibit VEGFR-2 ( } \text { IC }_{50}=0.47-6.29 \mu \mathrm{M}\right)\end{array}$ & [87] \\
\hline $\begin{array}{l}\text { ML model, based on } \\
\text { Laplacien-modified naïve Bayesian } \\
\text { classifiers. The ML model for EGFR } \\
\text { was coupled with a structure-based } \\
\text { technique regarding } \\
\text { the bromodomain }\end{array}$ & $\begin{array}{c}\text { Anticancer drug } \\
\text { discovery—target EGFR/BRD4 }\end{array}$ & $\begin{array}{l}\text { Two ML models for EGFR were } \\
\text { developed considering ECFP4 } \\
\text { based on a total of } \\
591,744 \text { unique kinase } \\
\text { compounds (one with } 3058 \text { active } \\
\text { molecules, } \mathrm{pIC}_{50} / \mathrm{pK}_{i} \geq 7 \text {, and } \\
\text { another with } 4785 \text { active } \\
\text { compounds, } \mathrm{pIC}_{50} / \mathrm{pK}_{i} \geq 6 \text { ). }\end{array}$ & $\begin{array}{l}\text { Area under ROC curve values of } 0.98 \\
\text { to } 0.99 \text { based on } 50 / 50 \text { training/test } \\
\text { set and assessed employing LOO-cv }\end{array}$ & $\begin{array}{l}\text { Virtual screening campaign employing a large } \\
\text { database (eMolecules }>6 \text { million compounds). } \\
\text { Among them, a first-in-class dual EGFR-BRD4 } \\
\text { inhibitor (compound 2870) was found (EGFR } \\
\mathrm{IC}_{50}=44 \mathrm{nM} \text {; ERBB2, ERBB4, and BRD4 } \\
\mathrm{IC}_{50}=8.73,24.2 \text {, and } 9.02 \mu \mathrm{M} \text {, respectively) }\end{array}$ & [89] \\
\hline
\end{tabular}


Table 1. Cont.

\section{AI Technique}

ML model based on a

GCNN algorithm

DL method

DNN model

ML models, employing naïve

Bayesian and RP techniques

Bayesian ML model

GENTRL

Statistical

Parameter

Outcomes

Ref

DeepMalaria antimalarial drug discovery

Discovery of novel antibiotic agents, possessing antibacterial profile

DNA gyrase to find broad-spectrum antibacterial agents

Antiviral research—Ebola virus

868 molecules viral pseudotype entry assay and the Ebola virus replication assay data

The model was generated using six data sets: (i) molecules from the ZINC database; (ii) inhibitors of DDR1 kinase; (iii) common kinase inhibitors (positive set); (iv) actives against non-kinase targets (negative set); (v) patent data of biological actives; (vi) 3D structures for DDR1 inhibitors

For de novo small molecule DDR1 kinase
Accuracy from $44.13 \%$ in the whole library to $87.75 \%$. Accuracy of $100 \%$ or all nanomolar active compound

rea under ROC curve of 0.896 considering the test data

The overall predictive accuracy, considering the training and test sets,

was greater than $80 \%$

Cross-validation showed ROC values greater than 0.8
Experimental validation-GENTRL allowed indication of several compounds for the synthesis, and the authors synthesized six lead compounds
The developed model was validated by predicting hit molecules from an additional chemical collection and a FDA-approved drug database. DeepMalaria identified all molecules showing nanomolar activity and $87.5 \%$ of chemicals with greater percentage of inhibition

Virtual screening of various chemical libraries.

From this screening step, they identify an existing drug, namely, halicin (SU-3327), showing

interesting bactericidal activity in vitro as well as in vivo. It was found to be effective against $M$. tuberculosis. Virtual screening of ZINC15

( $>100$ million compounds) provided eight further antibacterial agents, chemically unrelated to known antibiotics. ZINC000100032716 and ZINC000225434673 showed strong

broad-spectrum activity, overcoming a range of requent resistance factors

ML models used for virtual screening of a chemical library. The potential hits were

experimentally validated against DNA gyrase, $E$. bacteria. For compounds able to inhibit DNA gyrase, MIC values range between 1 and $32 \mu \mathrm{g} / \mathrm{mL}$, and the relative inhibition rates of inhibitors, range from $42 \%$ to $75 \%$ at $1 \mu \mathrm{M}$

Virtual screening campaign using the MicroSource library of drugs, for selecting possible antiviral compounds. Among the retrieved potential hit compounds, three promising antiviral candidates were found (quinacrine, pyronaridine, and tilorone $\mathrm{EC}_{50}=350,420$, and $230 \mathrm{nM}$, respectively, against Ebola virus replication).

Two molecules strongly inhibited DDR1 activity $\left(\mathrm{IC}_{50}=10-21 \mathrm{nM}\right)$, the other two compounds showed moderate potency $\left(\mathrm{IC}_{50}=0.278-1 \mu \mathrm{M}\right)$ 
Table 1. Cont.

AI Technique

Target

Three drug targets (sEH, a

hydrolase, ER $\alpha$, a nuclear

ML models

receptor, c-KIT, a kinase)

De novo design of small

DL and reinforcement learning DNN

molecules with desired profile molecules with desired profile,
and JAK2 as the target protein
The generative network was

trained with $\sim 1.5$ million

structures from the ChEMBL21 database
Models were trained on the DEL lection data for classifying molecules (over 2000)

\section{Statistical}

\section{Parameters}

Outcomes

Ref

Virtual screening of large chemical databases
( 88 million compounds). The outcomes revealed

that the technique is efficient, with a global hit

rate of $\sim 30 \%$ at $30 \mu \mathrm{M}$, discovering powerful

compounds $\left(\mathrm{IC}_{50}<10 \mathrm{nM}\right)$ for each drug target ReLeaSE was successfully applied for generating a series of libraries containing chemical entities

with a precise profile: (a) satisfactory

drug-likeness, regarding physchem properties, for which the authors chose $T_{\mathrm{m}}$ and $n$-octanol/water partition coefficient $(\log P)$; (b) desired biological partition coefficient $(\log P)$; (b) desired biological activity, for which the authors selected Janus
protein kinase 2 (JAK2) as the target protein

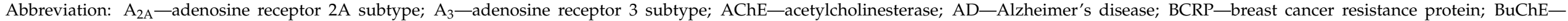

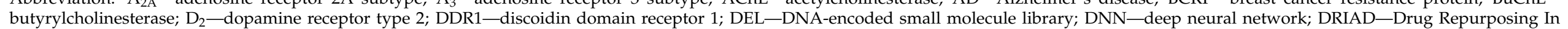

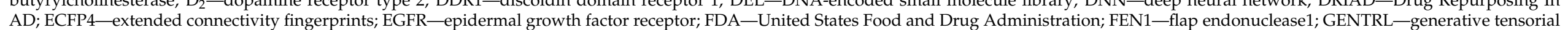

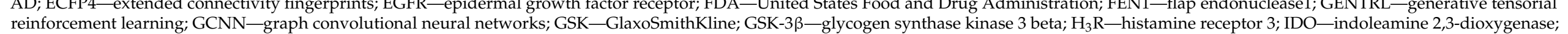

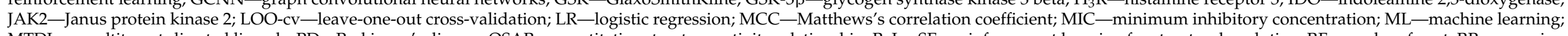

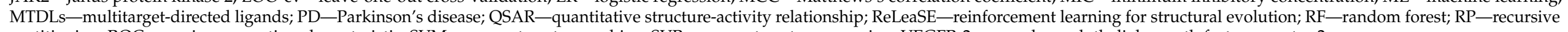
partitioning; ROC—receiver operating characteristic; SVM—support vector machine; SVR—support vector regression; VEGFR-2—vascular endothelial growth factor receptor 2.
} 
Table 2. Main examples of AI/ML in drug target prediction and biomarker identification.

\begin{tabular}{|c|c|c|c|c|c|}
\hline AI Technique & Target & Dataset & $\begin{array}{l}\text { Statistical } \\
\text { Parameters }\end{array}$ & Outcomes & Ref \\
\hline DL methodology deepDTnet & Multiple sclerosis & $\begin{array}{l}\text { DeepDTnet was generated } \\
\text { using } 732 \text { FDA-approved } \\
\text { for training }\end{array}$ & $\begin{array}{l}\text { Area under the ROC } \\
\quad \text { curve }=0.963\end{array}$ & $\begin{array}{c}\text { Topotecan was predicted as an inhibitor of } \\
\text { ROR- } \gamma \mathrm{t},\left(\mathrm{IC} \mathrm{C}_{50}=0.43 \mu \mathrm{M}\right) \text {, showing potential } \\
\text { therapeutic effects in multiple sclerosis, } \\
\text { being effective in reverting the pathological } \\
\text { phenotype in vivo in an EAE mouse model } \\
\text { at } 10 \mathrm{mg} / \mathrm{kg}\end{array}$ & {$[102]$} \\
\hline $\begin{array}{l}\text { ML-based approach } \\
\text { RF algorithm }\end{array}$ & $\begin{array}{l}\text { Druggability score of novel } \\
\text { unidentified drug targets }\end{array}$ & $\begin{array}{l}\text { The ML model included } \\
70 \text { features obtained from } \\
\text { drug targets, generating } \\
\text { 10,000 ML models using a } \\
\text { training set enclosing } \\
102 \text { complexes drug } \\
\text { targets / drugs, and a } \\
\text { "negative" set enclosing } \\
102 \text { non-drug targets }\end{array}$ & $\begin{array}{l}\text { The ML models discriminated } \\
\text { drug targets. The approach was } \\
\text { validated using an external test } \\
\text { set of } 277 \text { clinically relevant drug } \\
\text { targets (area under the ROC } \\
\text { curve of } 0.89 \text { ) }\end{array}$ & $\begin{array}{c}\text { The output reported in this work provided } \\
\text { new potential drug targets for developing } \\
\text { innovative anticancer drugs }\end{array}$ & [109] \\
\hline
\end{tabular}

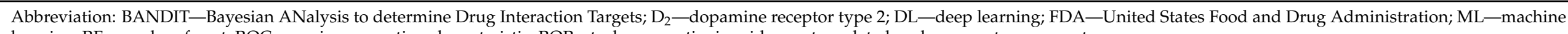
learning; RF-random forest; ROC—receiver operating characteristic; ROR- $\gamma \mathrm{t}$-human retinoic-acid-receptor-related orphan receptor-gamma $t$. 


\subsubsection{AI/ML in Quantitative Systems Pharmacology (QSP)}

Following the identification of prospective therapeutic drug targets, analysis must be performed to validate them. Computational approaches offer affordable, time-saving strategies to evaluate the likelihood that potential targets could provide an efficient method for treating a given disorder. Accordingly, a pivotal step in target validation is represented by the construction of a confidence interval for a given potential therapeutic hypothesis, employing quantitative systems pharmacology (QSP) models [110]. QSP is a stimulating and effective conjunction of biological pathways, pharmacology, and mathematical models for drug development. QSP possesses potential for providing a considerable impact on modern medicine as a result of the discovery and deployment of new molecular pathways and drug targets in the quest for innovative therapeutic agents and personalized medicine. The combination of these specialties is triggering substantial attention in pharma companies to expand predictions from a pharmacodynamic (PD) and pharmacokinetics (PK) perspective, and through improvements in computing capacity, QSP is currently capable of improving outcomes in the drug discovery trajectory. In fact, QSP models can combine information on PK/PD properties, biological processes of interest, and mechanisms of action, resulting from prior knowledge and available preclinical and clinical data, to quantitatively predict efficacy and safety responses over time and translate molecular data to clinical outcomes [111-114]. QSP provides a perfect quantitative framework for integrating different big data sources, including omics (i.e., proteomics, transcriptomics, metabolomics, and genomics) and imaging, the dimensionality of which can be reduced using ML methods. By allowing the identification of relevant association and data representations, the development of QSP platforms with higher granularity and enhanced predictive power can be further enhanced [115]. Moreover, the opportunity to implement a QSP platform with ML techniques to enhance the capacity to handle big data can offer great opportunities for systems pharmacology modeling. In fact, with the high availability of processed and organized data for building interpretable and actionable computational models, supporting decision making in the whole process of drug discovery and development, QSP can improve the reliability of predictions, providing more complex analysis, a better understanding of biomedical systems, and ultimately the design of optimized treatments. We report some examples regarding this approach.

In a recent study, Ramm and collaborators took advantage of systems biology methods coupled with multi-dimensional datasets and ML for identifying biomarkers to predict nephrotoxic molecules, for characterizing their mechanism of toxicity in vitro. The authors employed primary human kidney cells and used an approach based on systems biology, combining multidimensional datasets and ML for identifying biomarkers for predicting nephrotoxic molecules, along with the mechanism of toxicity. ML using the RF technique was applied for systematically identifying genes and imaging features from 46 different nephrotoxic compounds. From this analysis, the authors acquired information regarding changes in cell morphology as well as mRNA levels, finding and validating HMOX1 and SQSTM1 as nephrotoxic biomarkers. Furthermore, the RF algorithm was trained and validated using clinical observations of kidney toxicity and employed for nephrotoxicity classification (class labels as nontoxic $=0$ (10 instances, including 8 molecules, DMSO, and medium controls) or toxic $=1(38$ molecules) $)$. The developed computational model could discriminate nephrotoxic from non-nephrotoxic molecules and a hierarchical clustering approach, considering chemicals with an established mechanism of action, allowing the detection of the potential mechanisms of toxicity of drug candidates [116].

Notably, the individuation of appropriate and useful therapies for treating a given pathology is extremely important. Computational models can help with this issue, also providing the responsiveness of patients for a given treatment. In an interesting work, Song and coworkers reported the development and validation of a large-scale bidirectional generative adversarial network for predicting the tyrosine kinase inhibitor (TKI) response in patients with stage IV EGFR variant-positive non-small cell lung cancer. In the mentioned diagnostic/prognostic study were enrolled 465 patients, and the authors developed 
a DL semantic signature for predicting progression-free survival (PFS), which was built into the training group. The computational approach was validated by employing two external validation and two control groups, compared with the radiomics signature. Briefly, 342 subjects with stage IV EGFR variant-positive non-small cell lung cancer receiving EGFR-TKI therapy met the inclusion criteria. Of these, 145 patients from two hospitals ( $n=117$ and 28) were included in the training group, and the patients from two additional hospitals established two external validation groups (validation cohort 1: $n=101$; validation cohort 2: $n=96$ ). A total of 56 patients with advanced-stage EGFR variant-positive non-small cell lung cancer and 67 patients with advanced-stage EGFR wild-type non-small cell lung cancer who received first-line chemotherapy were included. A total of 90 subjects $(26 \%)$ receiving EGFR-TKI therapy with a high risk of rapid disease progression were detected by applying the DL semantic signature. When compared to other patients in validation groups, PFS dropped by $36 \%$ (hazard ratio, $2.13 ; 95 \%$ CI, $1.30-3.49 ; P=0.001$ ). When comparing the PFS of high-risk patients receiving EGFR-TKI treatment to chemotherapy groups, no substantial variations were detected (median PFS, 6.9 vs. 4.4 months; $P=0.08$ ). In terms of predicting tumor progression risk after EGFR-TKI therapy, clinical decisions based on the DL semantic signature led to better survival outcomes than those based on radiomics signatures across all risk probabilities by decision curve analysis [117]. Recently, $\mathrm{Lu}$ and collaborators described a significant ML approach based on the DL algorithm for predicting patient response time course from early data via neural-PK/PD modeling. Currently, analyses of patient response following doses of therapeutics are conducted employing standard PK/PD methods that require relevant human scientific expertise. Interestingly, DL has been applied to system pharmacology, as in the case of PK/PD models that directly learn the governing equations from data for predicting patient response time course, and for simulating the effects of unseen dosing regimens. Accordingly, the authors, in this new methodology, combined crucial pharmacological rules with neural ordinary differential equations. This neural-PK/PD model was used for analyzing the drug concentration and platelet response considering a clinical dataset comprising over 600 patients. In particular, the computational strategy was applied to predict drug concentration and platelet dynamics after treatment with trastuzumab emtansine (intravenous administration at $3.6 \mathrm{mg} / \mathrm{kg}$ once every three weeks) for human epidermal growth factor receptor 2 (HER2)-positive metastatic breast cancer in subjects failing treatment beforehand with trastuzumab and taxanes. The outcomes demonstrated that the computational model could predict patient responses, and simulate patient responses considering untested dosing regimens. These findings prove the potential of neural-PK/PD for automated predictive analytics of patient response time course, suggesting that the AI/ML approach can support clinical pharmacologists with the prospect, in the near future, to use neural-PK/PD as an advanced analytics tool for understanding and predicting drug concentration and response for dosing recommendation [118].

At the end of this section, day-by-day it is evident how AI has emerged in the field of drug discovery and development, being able to improve affordable and effective therapeutic treatments for common and emerging disorders, accelerating drug repurposing and minimizing the translational gap in drug development.

\subsection{Imaging, Biomarkers, Diagnosis, and Disease Progression}

\subsubsection{General Consideration}

With the growing accessibility to high-quality amounts of cell imaging data, there are currently relevant possibilities to use ML-based methods to aid researchers in cell image processing. In fact, the image features that are supposed to be crucial in producing predictions or diagnoses can be generally processed using ML algorithms. The latter offers the possibility of predictive, descriptive, and prescriptive assessment to acquire relevant information that would otherwise be impossible to obtain by human analysis, providing accurate medical diagnoses $[119,120]$. Accordingly, in recent years, numerous clinical investigations have enabled the use of $\mathrm{AI}$ in several fields, providing general pathological 
classification, risk evaluation, diagnosis, prognosis, and the prediction of appropriate therapy and possible responses to a given pharmacological treatment [121,122]. In particular, DL, a class of ML that employs ANN (CNN and recurrent neural networks (RNN)) resembling human cognitive capabilities, has proven undeniable superiority over conventional ML approaches owing to algorithm improvement, better processing hardware, and access to massive amounts of imaging data [123]. The successful incorporation of DL technology into normal clinical practice has determined that the diagnosis accuracy is comparable to that of healthcare experts. Furthermore, DL model integration provides additional advantages, including speed, efficiency, affordability, increased accessibility, and ethical behavior [120]. For these reasons, the FDA has approved the use of specific DL-driven diagnostic computational tools for clinical usage (Table 3) [124-126]. The application of AI encompasses several medical and biomedical fields, including radiology [127], gastroenterology $[128,129]$, neurology [130,131], ophthalmology [132,133], cardiology [134,135], dermatology [136], general pathology [137], oncology [138], healthcare [139,140], and clinical medicine $[141,142]$. 
Table 3. List of some examples of FDA-approved AI/ML-based solutions [124,126,138,143-145].

\begin{tabular}{|c|c|c|c|c|c|}
\hline Device/Algorithm (Company) & Type of Algorithm & Description & FDA Approval Number & Medical Field(s) & Date and Reference \\
\hline $\begin{array}{c}\text { Accipio Ix } \\
\text { (MaxQ-Al Ltd.), Tel Aviv, Israel }\end{array}$ & AI & $\begin{array}{c}\text { The tool is used for an automatic, rapid, highly accurate } \\
\text { identification and prioritization of suspected } \\
\text { intracranial hemorrhage }\end{array}$ & K182177 & $\begin{array}{l}\text { Radiology } \\
\text { Neurology }\end{array}$ & $\begin{array}{l}\text { October } 2018 \\
\quad[146]\end{array}$ \\
\hline $\begin{array}{c}\text { Advanced Intelligent Clear-IQ Engine (AiCE) } \\
\text { (Canon Medical Systems Corporation, } \\
\text { Ötawara, Japan) }\end{array}$ & Deep CNN & $\begin{array}{l}\text { AiCE system is used for reducing noise-boosting signals to } \\
\text { quickly deliver sharp, clear, and distinct images }\end{array}$ & K183046 & Radiology & $\begin{array}{l}\text { June } 2019 \\
\text { [147] }\end{array}$ \\
\hline $\begin{array}{l}\text { AI-Rad Companion (Cardiovascular) (Siemens } \\
\text { Medical Solutions USA, Inc., Malvern, PA, USA) }\end{array}$ & DL & $\begin{array}{l}\text { The software is used for detecting cardiovascular risks } \\
\text { from CT images }\end{array}$ & K183268 & Radiology & $\begin{array}{l}\text { October } 2019 \\
{[148,149]}\end{array}$ \\
\hline $\begin{array}{l}\text { AI-Rad Companion (Pulmonary) } \\
\text { (Siemens Medical Solutions USA, Inc., } \\
\text { Malvern, PA, USA) }\end{array}$ & DL & $\begin{array}{l}\text { The software is used for detecting lung nodules from } \\
\text { CT images }\end{array}$ & K183271 & Radiology & $\begin{array}{l}\text { July } 2019 \\
{[148,149]}\end{array}$ \\
\hline $\begin{array}{c}\text { AI Segmentation } \\
\text { (Varian Medical Systems, Inc., Crawley, UK) }\end{array}$ & AI & $\begin{array}{l}\text { The software is used for providing fast, accurate, and } \\
\text { intelligent contouring for improving the reproducibility of } \\
\text { structure delineation in radiation oncology }\end{array}$ & K203469 & $\begin{array}{l}\text { Radiology } \\
\text { Oncology }\end{array}$ & $\begin{array}{l}\text { April } 2021 \\
{[150]}\end{array}$ \\
\hline $\begin{array}{c}\text { AmCAD-UO } \\
\text { (AmCad BioMed Corporation, Taipei City, Taiwan) }\end{array}$ & AI & $\begin{array}{l}\text { The tool is used for detecting OSA in awake patients; it } \\
\text { can precisely scan upper airway and analyze the gap } \\
\text { between normal breathing and Müller Maneuver models }\end{array}$ & K180867 & Radiology & $\begin{array}{l}\text { December } 2018 \\
{[151]}\end{array}$ \\
\hline $\begin{array}{c}\text { AmCAD-US } \\
\text { (AmCad BioMed Corporation, Taipei City, Taiwan) }\end{array}$ & AI & $\begin{array}{c}\text { The tool is used to view and quantify ultrasound image } \\
\text { data of backscattered signals acquired from } \\
\text { ultrasound data }\end{array}$ & K162574 & Radiology & $\begin{array}{l}\text { May 2017 } \\
{[152]}\end{array}$ \\
\hline $\begin{array}{c}\text { AmCAD-UT Detection } 2.2 \\
\text { (AmCad BioMed Corporation, Taipei City, Taiwan) }\end{array}$ & AI & $\begin{array}{l}\text { The software is used for facilitating the detection, } \\
\text { visualization, and characterization of thyroid nodule } \\
\text { features on sonographic images }\end{array}$ & K180006 & Radiology & $\begin{array}{l}\text { August } 2018 \\
{[153,154]}\end{array}$ \\
\hline $\begin{array}{c}\text { AmCAD-UV } \\
\text { (AmCad BioMed Corporation, Taipei City, Taiwan) }\end{array}$ & AI & $\begin{array}{c}\text { The tool is used for classifying the ultrasonic color } \\
\text { intensity data from signals of flow Doppler } \\
\text { ultrasound images }\end{array}$ & K170069 & Radiology & $\begin{array}{l}\text { April } 2017 \\
{[155]}\end{array}$ \\
\hline $\begin{array}{c}\text { Arterys Cardio DL } \\
\text { (Arterys Inc., San Francisco, CA, USA) }\end{array}$ & DL & $\begin{array}{c}\text { The software is used for the analysis of cardiac } \\
\text { MRI images }\end{array}$ & K163253 & $\begin{array}{l}\text { Radiology } \\
\text { Cardiology }\end{array}$ & $\begin{array}{c}\text { January } 2017 \\
{[156]}\end{array}$ \\
\hline $\begin{array}{c}\text { Arterys Oncology DL } \\
\text { (Arterys Inc., San Francisco, CA, USA) }\end{array}$ & DL & $\begin{array}{l}\text { The software is used for measuring and tracking lesions } \\
\text { and nodules from MRI and CT images }\end{array}$ & K173542 & $\begin{array}{l}\text { Radiology } \\
\text { Oncology }\end{array}$ & $\begin{array}{c}\text { January } 2018 \\
{[157]}\end{array}$ \\
\hline $\begin{array}{c}\text { Arterys MICA } \\
\text { (Arterys Inc., San Francisco, CA, USA) }\end{array}$ & AI & $\begin{array}{l}\text { AI platform used for liver and lung cancer diagnosis from } \\
\text { MRI and CT images }\end{array}$ & K182034 & $\begin{array}{l}\text { Radiology } \\
\text { Oncology }\end{array}$ & $\begin{array}{c}\text { October } 2018 \\
\text { [158] }\end{array}$ \\
\hline $\begin{array}{l}\text { BladderScan Prime PLUS System } \\
\text { (Verathon Inc., Bothell, WA, USA) }\end{array}$ & DL & $\begin{array}{c}\text { The tool provides improved bladder volume } \\
\text { measurement accuracy }\end{array}$ & K172356 & Radiology & $\begin{array}{c}\text { September } 2017 \\
{[159]}\end{array}$ \\
\hline $\begin{array}{c}\text { Bone VCAR (BVCAR) } \\
\text { (GE Medical Systems SCS, Buc, France) }\end{array}$ & DL & $\begin{array}{l}\text { The tool is used for automated spine labeling (segments or } \\
\text { whole spine) from CT images }\end{array}$ & K183204 & Radiology & $\begin{array}{l}\text { April } 2019 \\
{[160]}\end{array}$ \\
\hline $\begin{array}{l}\text { Brainomix } 360^{\circ} \text { e-CTA } \\
\text { (Brainomix Limited, Oxford, UK) }\end{array}$ & AI & $\begin{array}{l}\text { The tool is used for automatically detecting LVO on } \\
\text { CT angiography }\end{array}$ & K192692 & Radiology & $\begin{array}{l}\text { May 2020 } \\
{[161,162]}\end{array}$ \\
\hline $\begin{array}{c}\text { BriefCase } \\
\text { (Aidoc Medical, Ltd., Tel Aviv, Israel) }\end{array}$ & DL & $\begin{array}{l}\text { The tool is used for detecting acute abnormalities across } \\
\text { the body, helping radiologists to prioritize life-threatening } \\
\text { cases, expediting patient care }\end{array}$ & K180647 & $\begin{array}{l}\text { Radiology } \\
\text { Emergency Medicine }\end{array}$ & $\begin{array}{l}\text { August } 2018 \\
{[163]}\end{array}$ \\
\hline
\end{tabular}


Table 3. Cont.

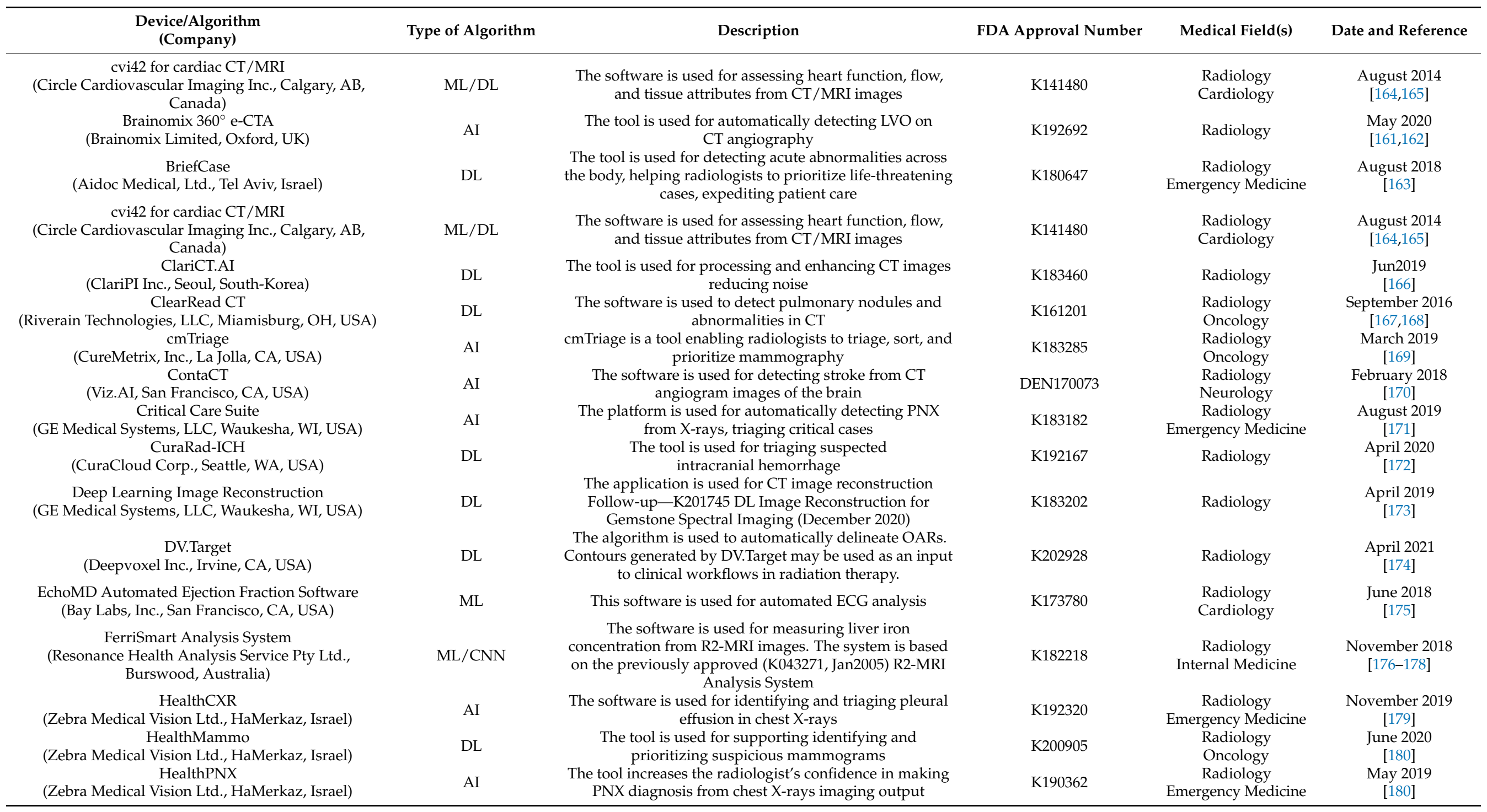


Table 3. Cont.

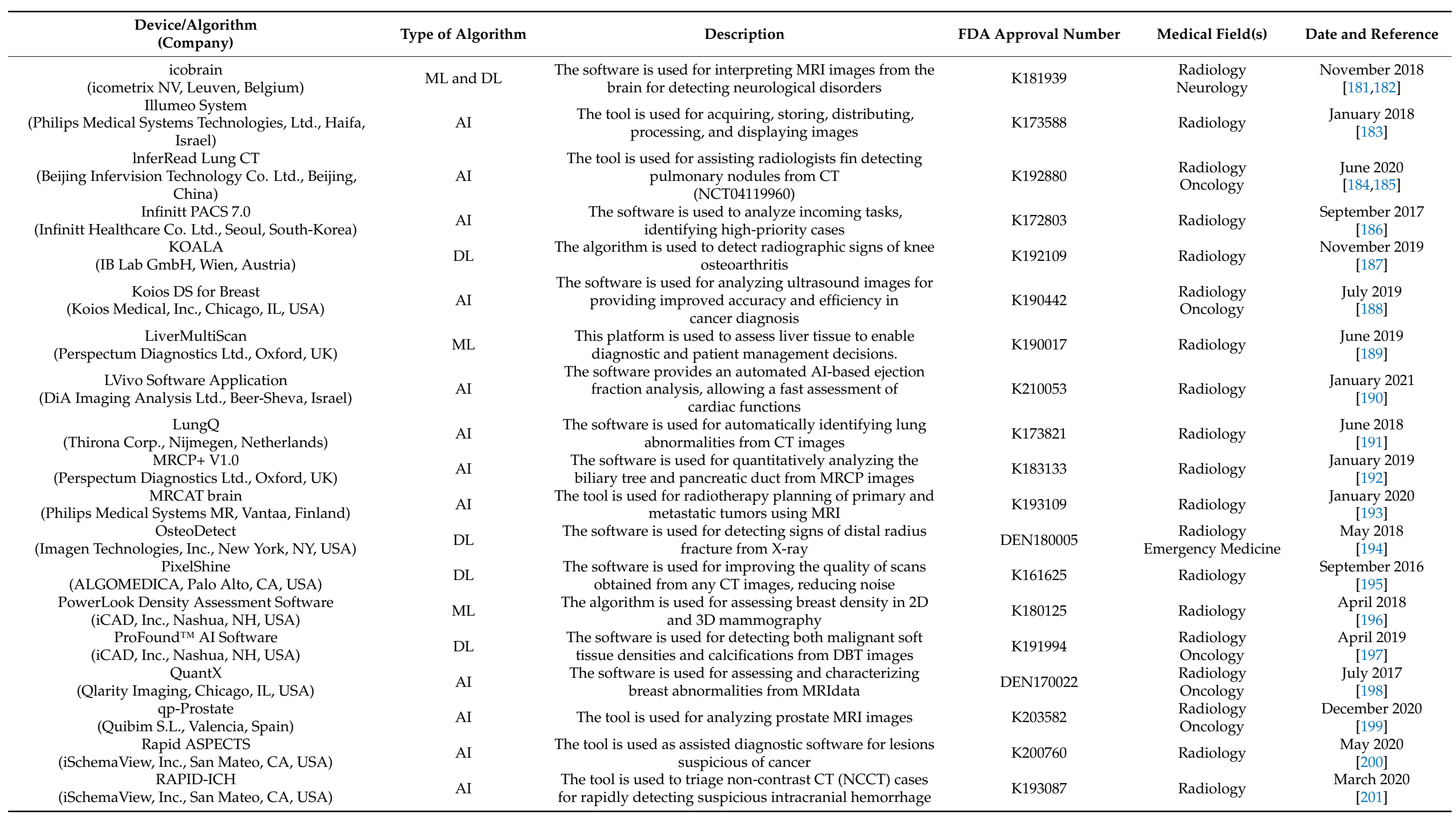


Table 3. Cont.

\begin{tabular}{|c|c|c|c|c|c|}
\hline $\begin{array}{l}\text { Device/Algorithm } \\
\text { (Company) }\end{array}$ & Type of Algorithm & Description & FDA Approval Number & Medical Field(s) & Date and Reference \\
\hline $\begin{array}{c}\text { RayCare 3.1 } \\
\text { (RaySearch Laboratories AB, Stockholm, Sweden) }\end{array}$ & $\mathrm{ML} / \mathrm{DL}$ & $\begin{array}{l}\text { The software is used for improving workflow efficiency } \\
\text { across different treatments in medical, radiation, and } \\
\text { surgical oncology to support decisions in the clinic }\end{array}$ & K200487 & $\begin{array}{l}\text { Radiology } \\
\text { Oncology }\end{array}$ & $\begin{array}{l}\text { June 2020 } \\
\text { [202] }\end{array}$ \\
\hline $\begin{array}{c}\text { RayStation } 10.1 \\
\text { (RaySearch Laboratories AB, Stockholm, Sweden) }\end{array}$ & ML & $\begin{array}{c}\text { The platform is used to automatically generate } \\
\text { treatment plans }\end{array}$ & K210645 & $\begin{array}{l}\text { Radiology } \\
\text { Oncology }\end{array}$ & $\begin{array}{l}\text { June } 2021 \\
\text { [203] }\end{array}$ \\
\hline $\begin{array}{l}\text { RBknee } \\
\text { (Radiobotics ApS, Copenaghen, Denmark) }\end{array}$ & ML & $\begin{array}{l}\text { The software is used for automatically identifying } \\
\text { osteoarthritis in the knees based on X-ray images }\end{array}$ & K203696 & Radiology & $\begin{array}{l}\text { August } 2021 \\
{[204]}\end{array}$ \\
\hline $\begin{array}{c}\text { Red Dot } \\
\text { (Behold.AI Technologies Ltd., London, UK) }\end{array}$ & AI & $\begin{array}{c}\text { The software is used for assessing PNX from chest } \\
\text { X-ray images }\end{array}$ & K191556 & Radiology & $\begin{array}{c}\text { January } 2020 \\
{[205]}\end{array}$ \\
\hline $\begin{array}{c}\text { StoneChecker } \\
\text { (Imaging Biometrics, LLC, Elm Grove, WI, USA) }\end{array}$ & AI & $\begin{array}{c}\text { The software is used with standard CT scans in people } \\
\text { with kidney stones for measuring stone parameters and to } \\
\text { inform clinical decisions }\end{array}$ & K191530 & Radiology & $\begin{array}{l}\text { June } 2019 \\
\text { [206] }\end{array}$ \\
\hline $\begin{array}{c}\text { StrokeViewer } \\
\text { (NiCo-Lab B.V., Amsterdam, Netherlands) }\end{array}$ & AI & $\begin{array}{l}\text { This tool is used for the localization and quantification of } \\
\text { stroke biomarkers from CT scans }\end{array}$ & K200873 & Radiology & $\begin{array}{l}\text { October } 2020 \\
\text { [207] }\end{array}$ \\
\hline $\begin{array}{c}\text { SubtleMR } \\
\text { (Subtle Medical, Inc., Menlo Park, CA, USA) }\end{array}$ & $\mathrm{CNN}$ & $\begin{array}{l}\text { The application is used for improving the quality of MRI } \\
\text { images increasing resolution and reducing noise }\end{array}$ & K191688 & Radiology & $\begin{array}{c}\text { September } 2019 \\
{[208]}\end{array}$ \\
\hline $\begin{array}{c}\text { SubtlePET } \\
\text { (Subtle Medical, Inc., Menlo Park, CA, USA) }\end{array}$ & DNN & The application is used for processing PET images & K182336 & Radiology & $\begin{array}{l}\text { November } 2018 \\
{[209]}\end{array}$ \\
\hline $\begin{array}{c}\text { syngo.CT Cardiac Planning } \\
\text { (Siemens Medical Solutions USA, Inc., Malvern, PA, } \\
\text { USA) }\end{array}$ & AI & $\begin{array}{l}\text { The software is used forenhancing CT images; analysis of } \\
\text { morphology and pathology of vascular and } \\
\text { cardiac structures }\end{array}$ & K200515 & Radiology & $\begin{array}{l}\text { March } 2020 \\
\quad[210]\end{array}$ \\
\hline $\begin{array}{c}\text { Transpara }{ }^{\mathrm{TM}} \\
\text { (Screenpoint Medical B.V., Nijmegen, Netherlands) }\end{array}$ & ML & $\begin{array}{l}\text { The software provides a support solution for } \\
\text { mammography, identifying suspicious areas in 2D and } \\
\text { 3D mammograms }\end{array}$ & K192287 & $\begin{array}{l}\text { Radiology } \\
\text { Oncology }\end{array}$ & $\begin{array}{l}\text { December } 2019 \\
\quad[211,212]\end{array}$ \\
\hline $\begin{array}{c}\text { Veolity } \\
\text { (MeVis Medical Solutions AG, Bremen, Germany) }\end{array}$ & ML & $\begin{array}{l}\text { The software is used to recognize even the subtlest } \\
\text { potential signs of lung cancer }\end{array}$ & K201501 & Radiology & $\begin{array}{c}\text { February } 2021 \\
{[213]}\end{array}$ \\
\hline $\begin{array}{l}\text { Workflow Box including DCLExpert } \\
\text { (Mirada } \\
\text { (Medical Ltd., Oxford, UK) }\end{array}$ & AI & $\begin{array}{l}\text { The software is used for autocontouring organs for cancer } \\
\text { treatment planning }\end{array}$ & K181572 & Radiology & $\begin{array}{l}\text { July } 2018 \\
\text { [214] }\end{array}$ \\
\hline $\begin{array}{c}\text { AI-ECG Platform } \\
\text { (Shenzhen Carewell Electronics, Ltd., Shenzhen, } \\
\text { China) }\end{array}$ & AI & $\begin{array}{l}\text { AI platform for assisting physicians in measuring and } \\
\text { interpreting ECG }\end{array}$ & K180432 & Cardiology & $\begin{array}{l}\text { November } 2018 \\
{[215]}\end{array}$ \\
\hline $\begin{array}{c}\text { AI-ECG Tracker } \\
\text { (Shenzhen Carewell Electronics, Ltd., Shenzhen, } \\
\text { China) }\end{array}$ & AI & $\begin{array}{l}\text { The tool is used for improving the detection efficiency of } \\
\text { non-persistent arrhythmias (irregular heartbeats) }\end{array}$ & K200036 & Cardiology & $\begin{array}{l}\text { March } 2020 \\
\quad[216]\end{array}$ \\
\hline $\begin{array}{c}\text { BioFlux Device } \\
\text { (Biotricity Inc., Redwood City, CA, USA) }\end{array}$ & AI & The tool is used for detecting arrhythmias & K172311 & Cardiology & $\begin{array}{c}\text { December } 2017 \\
{[217]}\end{array}$ \\
\hline $\begin{array}{l}\text { EchoGo Core } \\
\text { (Ultromics Ltd., Oxford, UK) }\end{array}$ & ML & $\begin{array}{l}\text { The application is used to automatically evaluate cardiac } \\
\text { functions from echocardiography, enabling physicians to } \\
\text { diagnose heart failure and coronary artery disease }\end{array}$ & K191171 & Cardiology & $\begin{array}{l}\text { November } 2019 \\
{[218]}\end{array}$ \\
\hline $\begin{array}{c}\text { Eko Analysis Software } \\
\text { (Eko Devices Inc., Oakland, CA, USA) }\end{array}$ & ANN & $\begin{array}{l}\text { The software is used for detecting suspected murmurs in } \\
\text { the heart sounds and atrial fibrillation from ECG data }\end{array}$ & K192004 & Cardiology & $\begin{array}{c}\text { January } 2020 \\
{[219]}\end{array}$ \\
\hline $\begin{array}{c}\text { eMurmur ID } \\
\text { (CSD Labs GmbH, Graz, Austria) }\end{array}$ & ML & $\begin{array}{l}\text { The software is used to understand, identify, and detect } \\
\text { heart murmurs }\end{array}$ & K181988 & Cardiology & $\begin{array}{l}\text { April } 2019 \\
{[220]}\end{array}$ \\
\hline
\end{tabular}


Table 3. Cont.

\begin{tabular}{|c|c|c|c|c|c|}
\hline $\begin{array}{l}\text { Device/Algorithm } \\
\text { (Company) }\end{array}$ & Type of Algorithm & Description & FDA Approval Number & Medical Field(s) & Date and Reference \\
\hline $\begin{array}{c}\text { KardiaAI } \\
\text { (AliveCor, Inc., Mountain View, CA, USA) }\end{array}$ & AI & $\begin{array}{c}\text { The tool is used for enhancing cardiac MRI to improve } \\
\text { diagnosis of heart disorders }\end{array}$ & K181823 & Cardiology & $\begin{array}{l}\text { November } 2019 \\
{[221]}\end{array}$ \\
\hline $\begin{array}{c}\text { KOSMOS } \\
\text { (EchoNous Inc., Redmond, WA, USA) }\end{array}$ & DL & $\begin{array}{l}\text { This tool combining ultrasound with DL is used for } \\
\text { clinical assessment of the heart, lungs, and abdomen }\end{array}$ & K193518 & Cardiology & $\begin{array}{c}\text { March } 2020 \\
{[222]}\end{array}$ \\
\hline $\begin{array}{l}\text { Ventripoint Medical System Plus (VMS+) } 3.0 \\
\text { (Ventripoint Diagnostics Ltd., Toronto, ON, Canada) }\end{array}$ & AI & $\begin{array}{l}\text { The tool is used for measuring whole heart function using } \\
\text { conventional ultrasound } \\
\text { (NCT01557582) }\end{array}$ & K191493 & Cardiology & $\begin{array}{l}\text { October } 2019 \\
{[223]}\end{array}$ \\
\hline $\begin{array}{c}\text { Altoida } \\
\text { (Altoida, Inc., Washington, DC, USA) }\end{array}$ & ML & $\begin{array}{l}\text { The software is used for detecting AD up to } 10 \text { years prior } \\
\text { to the onset. ML is used for classifying patients' risk of } \\
\text { MCI due to AD (NCT02843529) }\end{array}$ & FDA-ClassII & Neurology & $\begin{array}{l}\text { August } 2021 \\
{[224,225]}\end{array}$ \\
\hline $\begin{array}{l}\text { BrainScope Ahead } 100 \\
\text { (Brainscope Company, Inc., Bethesda, MD, USA) }\end{array}$ & AI & $\begin{array}{l}\text { The software is used for interpreting the structural } \\
\text { condition of the patient's brain after head injury from } \\
\text { EEG data }\end{array}$ & DEN140025 & Neurology & $\begin{array}{l}\text { November } 2014 \\
{[226]}\end{array}$ \\
\hline $\begin{array}{c}\text { Cognoa ASD Diagnosis Aid } \\
\text { (Cognoa, Inc., Palo Alto, CA, USA) }\end{array}$ & ML & The software is used for evaluating patients at risk of ASD & DEN200069 & Neurology & $\begin{array}{l}\text { June } 2021 \\
\text { [227] }\end{array}$ \\
\hline $\begin{array}{l}\text { complete control system gen2 } \\
\text { (Coapt, LLC, Chicago, IL, USA) }\end{array}$ & $\mathrm{AI} / \mathrm{ML}$ & $\begin{array}{l}\text { The platform provides a human-bionic interface that } \\
\text { learns and adapts to users, giving them unrivaled control } \\
\text { of their prosthetic arms }\end{array}$ & K191083 & Neurology & $\begin{array}{l}\text { April } 2019 \\
{[228]}\end{array}$ \\
\hline $\begin{array}{c}\text { EnsoSleep } \\
\text { (EnsoData, Inc., Madison, WI, USA) }\end{array}$ & AI & $\begin{array}{c}\text { The application assists clinicians in the diagnosis of } \\
\text { sleep disorders }\end{array}$ & K162627 & Neurology & $\begin{array}{l}\text { March } 2017 \\
\quad[229]\end{array}$ \\
\hline $\begin{array}{c}\text { Clarus } 700 \\
\text { (Carl Zeiss Meditec Inc., Dublin, CA, USA) }\end{array}$ & DL & $\begin{array}{l}\text { The algorithm is applied to diagnosing and monitoring } \\
\text { retina disorders }\end{array}$ & K191194 & Ophthalmology & $\begin{array}{l}\text { May } 2019 \\
{[232]}\end{array}$ \\
\hline $\begin{array}{c}\text { EyeArt } \\
\text { (EyeNuk, Inc., Woodland Hills, CA, USA) }\end{array}$ & AI & $\begin{array}{c}\text { The software is used as a screening tool for detecting } \\
\text { diabetic retinopathy }\end{array}$ & K200667 & Ophthalmology & $\begin{array}{l}\text { March 2020 } \\
{[233,234]}\end{array}$ \\
\hline $\begin{array}{c}\text { IDx } \\
\text { (Digital Diagnostics Inc. -IDx LLC., Coralville, IA, } \\
\text { USA) }\end{array}$ & AI & The software is used for detecting diabetic retinopathy & DEN180001 & Ophthalmology & $\begin{array}{c}\text { January } 2018 \\
{[235,236]}\end{array}$ \\
\hline $\begin{array}{c}\text { DreaMed Advisor Pro } \\
\text { (DreaMed Diabetes, Ltd., Petah Tikva, Israel) }\end{array}$ & AI & $\begin{array}{l}\text { The application is used for automatically determining the } \\
\text { optimal therapy to maintain balanced glucose levels }\end{array}$ & DEN170043 & Endocrinology & $\begin{array}{l}\text { June } 2018 \\
\text { [237] }\end{array}$ \\
\hline $\begin{array}{c}\text { Guardian Connect System } \\
\text { (Medtronic Minimed, Northridge, CA, USA) }\end{array}$ & AI & $\begin{array}{l}\text { The application is used with diabetic patients for } \\
\text { monitoring blood glucose content, predicting changes }\end{array}$ & P160007 & Endocrinology & $\begin{array}{l}\text { March } 2018 \\
{[238]}\end{array}$ \\
\hline $\begin{array}{c}\text { APAS Independence } \\
\text { (Clever Culture Systems AG, Bäch, Switzerland) }\end{array}$ & $\mathrm{AI} / \mathrm{ML}$ & $\begin{array}{l}\text { The tool is used to automate culture plate imaging, } \\
\text { analysis, and interpretation }\end{array}$ & K183648 & Microbiology & $\begin{array}{l}\text { September } 2019 \\
{[239,240]}\end{array}$ \\
\hline $\begin{array}{c}\text { NightOwl } \\
\text { (Ectosense nv, Leuven, Belgium) }\end{array}$ & AI & $\begin{array}{l}\text { parameters for evaluating sleep-related breathing } \\
\text { disorders of patients suspected of sleep apnea } \\
\text { (NCT03774199; NCT04194073) }\end{array}$ & K191031 & Anesthesiology & $\begin{array}{l}\text { March } 2020 \\
\quad[241]\end{array}$ \\
\hline
\end{tabular}


Table 3. Cont.

\section{Device/Algorithm} (Company)

NuVasive Pulse System

(NuVasive, Inc., San Diego, CA. USA) Sight OLO

(Sight Diagnostics Ltd., Tel Aviv, Israel)

$$
\mathrm{SOZO}
$$

(ImpediMed Ltd., Carlsbad, CA, USA)

wheezo WheezeRate Detector

(Respiri Ltd., Melbourne, Australia)

\section{Description}

Type of Algorithm

The tool is used during spinal surgery, neck dissection and thoracic surgeries, improving surgical procedures The algorithm is used for inspecting blood samples (NCT03595501)

The tool is use for the clinical assessment of unilateral lymphedema, combining BIS with AI to create a rapid, non-invasive scan of a person's body

The tool is used for asthma management and

$$
\text { remote monitoring }
$$

ML
Date and Reference

\section{FDA Approval Number}

Surgery

June 2018
$[242]$
November 2019
$[243,244]$

K190898

Hematology

[243,244]
K190529

Gastroenterology Urology

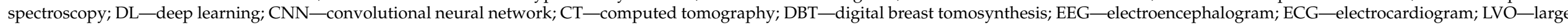

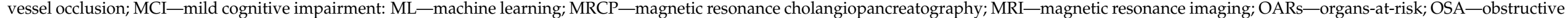
sleep apnea; PET—positron emission tomography; PNX—-pneumothorax. 


\subsubsection{Basic Research}

In this section, we illustrate some relevant and representative examples of how AI can be an added value in translational medicine, starting from research laboratories to clinical practice, speeding up the understanding of disorders (targets involved, phatophysiological mechanisms, etc.) and the translation of acquired knowledge in clinical medicine. For example, in medical/cellular imaging, ML-based methods hold great promise. Considering cell microscopy and histopathology, observation of the slides is often complicated, such that a pathologists' interpretation might be inconsistent, making histopathological diagnoses problematic [247]. Conventional approaches (e.g., microscopic/biological inspection of a sample) have limitations, reducing the possibility of discovering particular biomarkers, genomic driver mutations, and patterns within a cell's subcellular apparatus [248]. Accordingly, with the aid of ML, unravelling disease heterogeneity through enhancing the cellular profiling of specific morphological features is becoming progressively possible. ML approaches are able to improve sample categorization, allowing the acquisition of undisclosed disease characteristics that cannot be identified by humans alone (Table 4). To this end, Simm and collaborators described a fascinating approach in which an ML-based method was employed for predicting the activity of a given compound from images. The interesting idea starts with the evidence that large-scale assays (e.g., high-throughput screening) for the drug discovery pipeline are costly, time-consuming, and frequently unfeasible, mainly for the growing number of relevant physiological systems needing primary cells, organoids, or entire organisms, as well as pricey or rare reagents. The authors assumed that data from only high-throughput imaging (HTI) assay could be repurposed for predicting the bioactivities of molecules in other assays, similar to those that target different biological processes or pathways. For that purpose, they developed a protocol for predicting the activity of compounds in several orthogonal tests. In the first step, the researchers extracted a large set of image-based fingerprints of morphological descriptions for each molecule (considering the three-channel glucocorticoid receptor (GCR) as a target for the HTI assay employed in the evaluation, the authors obtained 842-dimensional feature vectors per cell). The second step consisted of introducing known activity data for orthogonal assays of interest on the considered molecules. Finally, by using the supervised ML approach, they trained models, selecting the one that showed higher predictivity. The resulting ML model was successfully used for selecting novel chemical entities for biological evaluation [249]. Another interesting study was conducted by Nassar and colleagues. They reported an MLbased method (evaluating six ML algorithms: AdaBoost, Gradient Boosting (GB), k-NN, $\mathrm{RF}$, and SVM) for classifying white blood cells (WBCs). Currently, WBC count, a method for assessing the immune system status of a person, requires a flow cytometer and fluorescent markers. Obviously, for accomplishing this process, various steps for sample preparation are required. By using the proposed label-free approach only, employing an imaging flow cytometer combined with ML methods, unstained WBCs were classified. The developed model showed good scores, being also able to discriminate B and T lymphocytes. The approach was validated by performing WBC analyses from unstained samples collected from 85 donors. Notably, the described approach allows an extremely precise classification of WBCs while avoiding cell disruption and leaving marker channels open to address further biological issues. In the end, the proposed method enables the use of ML for liquid biopsy, applying powerful information regarding cell morphology for several diagnostics of primary blood, such as, for example, the detection of tumor products or circulating tumor cells in the blood [250]. Coudray and colleagues applied ML algorithms for classifying and predicting mutations from histopathological images belonging to non-small cell lung cancer. In fact, the visual inspection represents the elected methodology for assessing stage, type, and subtype of lung cancers. Expert pathologists can distinguish adenocarcinoma (LUAD) and squamous cell carcinoma (LUSC) by visual inspection. The authors presented an ML approach based on deep CNN trained on whole-slide images acquired from The Cancer Genome Atlas for accurately and automatically classifying them into LUAD, LUSC, or normal lung tissue. The performance of the methodology is equivalent to that of pathol- 
ogists, showing an average area under the ROC curve of 0.97 . The in silico model was validated on independent datasets of frozen tissues, formalin-fixed paraffin-embedded tissues, and biopsies. Additionally, the network was also trained for predicting ten of the most frequently mutated genes in LUAD. Six of them (STK11, EGFR, FAT1, SETBP1, KRAS, and TP53) can be predicted from pathology images, with a significant area under the ROC curve (0.733-0.856) as determined on a held-out population. Remarkably, a similar approach based on ML models could aid pathologists in detecting gene mutations related to cancer subtypes [251]. Moreover, ML-based approaches can assist in identifying specific biomarkers involved in a given disease. The most fruitful computer-based approaches were recently well reviewed [6,252]. To understand the task, we report some examples highlighting ML approaches in this field. Kang and collaborators used the python package sklearn for building an ML-based computational model, employing the SVM technique, that executed 10-fold cross-validation to implement a diagnostic tool for identifying the lung cancer risk of suspected cases. The authors performed an inclusive assessment of results from genetic analysis and critical clinical data regarding patients affected by lung cancer to develop a model able to diagnose early lung cancer, also indicating tumor risks. They considered tissues from samples of patients with lung cancer and tissue from healthy persons for a total of 70 pairs. The authors evaluated the methylation rates of six genes (FHIT, p16, MGMT, RASSF1A, APC, DAPK) in lung cancer patients, as well as the critical clinical data and tumor marker concentrations of these patients. The SVM model was validated by calculating the area under the ROC curve and other statistical parameters. Based on these validation data (area under the ROC curve of 0.963 , sensitivity of 0.900 , specificity of 0.971 , and accuracy of 0.936 ), the scientists proved the validity of the developed method, highlighting the crucial role of ML models as diagnostic tools for the early diagnosis of cancers that can contribute to increase the survival rate of patients [253]. 
Table 4. Main examples of AI/ML in basic research.

\begin{tabular}{|c|c|c|c|c|c|}
\hline AI Technique & Target & Dataset & Statistical Parameters & Outcomes & Ref \\
\hline $\begin{array}{l}\text { ML-based method } \\
\text { DNN } \\
\text { algorithm }\end{array}$ & $\begin{array}{l}\text { Predicting the activity of a given } \\
\text { compound from images }\end{array}$ & $\begin{array}{l}\text { Image-based fingerprints of } \\
\text { morphological descriptions for } \\
\text { each molecule considering GCR } \\
\text { as a target for HTI assays used. A } \\
\text { total of } 842 \text {-dimensional feature } \\
\text { vectors per cell related to activity } \\
\text { data for selected orthogonal } \\
\text { assays. Supervised ML for } \\
\text { training models }\end{array}$ & $\begin{array}{l}\text { Area under the ROC curve }>0.9 \text { as threshold for } \\
\text { selecting the best performing models }\end{array}$ & $\begin{array}{l}\text { The resulting ML model was } \\
\text { successfully used for selecting } \\
\text { novel chemical entities for } \\
\text { biological evaluation }\end{array}$ & [249] \\
\hline $\begin{array}{l}\text { ML-based method (evaluating six } \\
\text { ML algorithms: AdaBoost, GB, } \\
\text { k-NN, RF, and SVM) }\end{array}$ & $\begin{array}{c}\text { Classifying WBC for assessing } \\
\text { the immune system status of } \\
\text { a person }\end{array}$ & $\begin{array}{l}\text { By using the proposed label-free } \\
\text { approach only employing an } \\
\text { imaging flow cytometer } \\
\text { combined with ML methods, } \\
\text { unstained WBCs were classified }\end{array}$ & $\begin{array}{l}\text { The developed model discriminated B and T } \\
\text { lymphocytes. Validation was achieved performing } \\
\text { WBC analyses from unstained samples from } \\
85 \text { donors. The approach allows a precise classification } \\
\text { of WBC avoiding cell disruption, leaving marker } \\
\text { channels open to address further biological issues }\end{array}$ & $\begin{array}{l}\text { The proposed method enables } \\
\text { the use of ML for liquid biopsy, } \\
\text { applying the powerful } \\
\text { information in cell morphology } \\
\text { for several diagnostics (e.g., } \\
\text { detection of tumor products or } \\
\text { circulating tumor cells in } \\
\text { the blood }\end{array}$ & [250] \\
\hline $\begin{array}{l}\text { ML algorithms } \\
\text { CNN }\end{array}$ & $\begin{array}{c}\text { Classifying and predicting } \\
\text { mutations from histopathological } \\
\text { images from non-small cell lung } \\
\text { cancer into LUAD, LUSC or } \\
\text { normal lung tissue }\end{array}$ & $\begin{array}{l}\text { Whole-slide images acquired } \\
\text { from The Cancer Genome Atlas. } \\
\text { The network was also trained for } \\
\text { predicting most frequently } \\
\text { mutated genes in LUAD (STK11, } \\
\text { EGFR, FAT1, SETBP1, KRAS, and } \\
\text { TP53) }\end{array}$ & $\begin{array}{l}\text { The ML model performance was equivalent to that of } \\
\text { pathologists (area under the ROC curve = 0.97). } \\
\text { Validation using independent datasets of frozen } \\
\text { tissues, formalin-fixed paraffin-embedded tissues and } \\
\text { biopsies. Mutated genes in LUAD correctly predicted } \\
\text { from pathology images (area under the ROC curve } \\
0.733-0.856 \text { ) }\end{array}$ & $\begin{array}{l}\text { Aid pathologists in detecting } \\
\text { gene mutations related to } \\
\text { cancer subtypes }\end{array}$ & [251] \\
\hline $\begin{array}{l}\text { ML-based model, } \\
\text { SVM algorithm }\end{array}$ & $\begin{array}{l}\text { Implementing a diagnostic tool } \\
\text { for identifying lung cancer risk of } \\
\text { suspected cases }\end{array}$ & $\begin{array}{l}\text { Tissues from samples of patients } \\
\text { with lung cancer and tissue from } \\
\text { healthy persons (70 pairs). } \\
\text { Evaluation of the methylation } \\
\text { rates of six genes (FHIT, p16, } \\
\text { MGMT, RASSF1A, APC, DAPK) } \\
\text { in lung cancer patients, the } \\
\text { critical clinical data, tumor } \\
\text { marker concentrations }\end{array}$ & $\begin{array}{l}\text { Area under the ROC curve of } 0.963 \text {, sensitivity of } \\
0.900 \text {, specificity of } 0.971 \text {, and accuracy of } 0.936\end{array}$ & $\begin{array}{l}\text { ML models as diagnostic tools for } \\
\text { the early diagnosis of cancers } \\
\text { that can contribute to increasing } \\
\text { the survival rate of patients }\end{array}$ & [253] \\
\hline
\end{tabular}

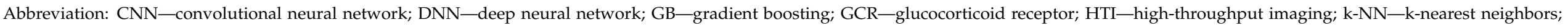

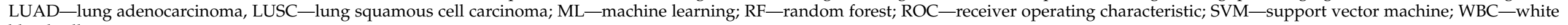
blood cell. 


\subsubsection{AI, Imaging and Ophthalmology}

In the context of imaging in diagnosis and disease progression, applying ML-based techniques, ophthalmology is one of the medical fields in which these computational approaches have been successfully employed [133]. In fact, AI principally based on DL methods has been used to detect several ocular disorders, including retinopathy of prematurity [254], diabetic retinopathy [255,256], macular edema [257,258], age-related macular degeneration [259,260], and glaucoma [261-263], using fundus images, optical coherence tomography (OCT), and visual fields. Screening, diagnosis, and monitoring of major eye disorders for patients in primary care might be achievable using DL in ocular imaging combined with telemedicine. Briefly, we report here some representative examples of how ML can revolutionize diagnostics, improving the quality of diagnosis, reducing potential medical errors and the workload of medical staff, and also saving the time of the patients examined (Table 5). Recently, Dai and coworkers reported the development of an intriguing screening platform for detecting diabetic retinopathy. It is well established that retinal screening has a tremendous impact on the early diagnosis of retinopathy to start effective treatments to avoid vision loss, slowing down the progression of the disorder. For facilitating the screening procedure, they used an ML approach based on DL algorithms for developing a computational tool, namely, DeepDR (DL Diabetic Retinopathy). DeepDR is a transfer-learning-aided multi-task network for evaluating retinal image features, retinal lesions, and diabetic retinopathy grades. This evaluation allows the detection of early-tolate stages of diabetic retinopathy. DeepDR was generated considering 666,383 fundus images from 173,346 patients, and it is trained for real-time image quality evaluation, lesion detection, and grading; 466,247 fundus images from 121,342 patients (70\%) affected by diabetes were randomly included in the training set, while the evaluation was conducted considering 52,004 patients (30\%) for a local validation set consisting of 200,136 fundus images and three external datasets containing 209,322 images. The results show an area under ROC curves of $0.901,0.941,0.954$, and 0.967 regarding the detection of microaneurysms, cotton-wool spots, hard exudates, and hemorrhages, respectively, while the grading of diabetic retinopathy as mild, moderate, severe, and proliferative accomplishes a significant area under the ROC curves $(0.943,0.955,0.960$, and 0.972 , respectively). Finally, the statistical parameters, considering the external validation, ranged from 0.916 to 0.970 (area under the ROC curves). In summary, DeepDR showed significant accuracy and high sensitivity in detecting diabetic retinopathy from early-to-late stages [255]. Asaoka and colleagues reported an ML approach based on deep and transfer learning for an accurate diagnosis regarding early-onset glaucoma using October images [263]. The DL model was built starting from 4316 October images from 1565 eyes from patients suffering from glaucoma and 193 normal eyes, used as a pre-training dataset. A smaller set of October images was used to train the model (94 eyes from patient with early glaucoma and 84 healthy eyes). The independent dataset employed as a test set for assessing the diagnostic performance of the developed model comprised 114 eyes from 114 patients at early stages of glaucoma and 82 eyes from 82 healthy people. In particular, a DL classifier based on CNN was employed in the reported study, and the input features were $8 \times 8$ grid macular retinal nerve fiber layer thickness and ganglion cell complex layer thickness from October images. Diagnostic performances were assessed using the test set and applying RF and the SVM algorithm. The results show that the DL model displayed an area under the ROC curve of $93.7 \%$, considerably decreasing (to 76.6 and $78.8 \%$ ) with no pre-training procedure, suggesting a relevant sensitivity and specificity of the DL model to diagnose glaucoma, highlighting the robustness of the proposed approach. Accordingly, also in the reported case is underlined that the use of ML approaches can offer a significant improvement in diagnostic performance, assisting clinicians in making a decision [263]. Finally, another interesting approach was conducted by Zhang and collaborators. They used October images of the fundus retina for generating and validating an ML-based model as a diagnostic model for diabetic macular edema (DME). Concisely, the authors used 38,057 October images (drusen, choroidal neovascularization (CNV), DME, and healthy) in a multiscale transfer-learning 
algorithm model by using the CNN technique. This computational-based tool consisted of two steps (self-enhancement and disease detection). The self-enhancement model was built using a multiscale feature-learning method for detecting and extracting the frame of the diagnostic target. Next, the enhanced data were employed to generate a disease diagnostic model that combined transfer-learning knowledge. The data were initially processed by convolutional and pooling layers for extracting characteristics hidden in the original data. Lastly, these features were used in a classification step for automatically determining the type of disorder. In the training set were enclosed 37,457 samples (9891 cases $(26.41 \%)$ of CNV, 9633 cases $(25.72 \%)$ of DME, 7975 cases $(21.29 \%)$ of drusen, and 9958 healthy cases $(26.58 \%)$ ), while 600 samples (150 cases $(25 \%)$ of CNV, 150 cases $(25 \%)$ of DME, 150 cases $(25 \%)$ of drusen and 150 healthy cases $(25 \%))$ comprised the validation set. The statistical parameters (accuracy, precision, sensitivity, and specificity) of the model were evaluated as well as the parameters for assessing the performance of the ML-based model from the perspective of clinical application. The developed computational tool showed $94.5 \%$ accuracy, $97.2 \%$ precision, $97.7 \%$ sensitivity, and $97 \%$ specificity in the independent testing dataset. Notably, the developed model based on a multiscale transfer-learning algorithm can accurately employ October images for assessing the health of patients, automatically and accurately diagnosing several eye health conditions. Such an approach could help clinicians by improving the effectiveness of therapies, reducing the disability ratio of severe disorders [258]. 
Table 5. Main examples of AI/ML in ophthalmology.

\begin{tabular}{|c|c|c|c|c|c|}
\hline AI Technique & Target & Dataset & $\begin{array}{l}\text { Statistical } \\
\text { Parameters }\end{array}$ & Outcomes & Ref \\
\hline $\begin{array}{l}\text { ML approach based on DL } \\
\text { algorithms }\end{array}$ & $\begin{array}{l}\text { Development of DeepDR, an } \\
\text { intriguing screening platform } \\
\text { for detecting } \\
\text { diabetic retinopathy }\end{array}$ & $\begin{array}{l}\text { DeepDR was generated considering } \\
666,383 \text { fundus images ( } 173,346 \text { patients), } \\
\text { and it was trained for real-time image } \\
\text { quality valuation, lesion detection and } \\
\text { grading by } 466,247 \text { fundus images from } \\
\text { 121,342 patients with diabetes. The } \\
\text { evaluation was conducted considering } \\
52,004 \text { patients. Validation set: } \\
\text { 200,136 fundus images, three external } \\
\text { datasets, } 209,322 \text { images }\end{array}$ & $\begin{array}{c}\text { Area under ROC curves of } 0.901,0.941, \\
0.954 \text {, and } 0.967 \text { regarding the detection } \\
\text { of microaneurysms, cotton-wool spots, } \\
\text { hard exudates, and hemorrhages, } \\
\text { respectively. Area under the ROC curves } \\
\text { of } 0.943,0.955,0.960 \text {, and } 0.972, \\
\text { regarding the grading of diabetic } \\
\text { retinopathy (mild, moderate, severe, and } \\
\text { proliferative). External validation, area } \\
\text { under the ROC curves ranging from } \\
0.916 \text { to } 0.970\end{array}$ & $\begin{array}{l}\text { DeepDR showed significant } \\
\text { accuracy and high sensitivity } \\
\text { in detecting diabetic } \\
\text { retinopathy from } \\
\text { early-to-late stages }\end{array}$ & [255] \\
\hline $\begin{array}{l}\text { ML approach based on deep } \\
\text { and transfer learning }\end{array}$ & $\begin{array}{l}\text { Diagnosis regarding } \\
\text { early-onset glaucoma using } \\
\text { October images }\end{array}$ & $\begin{array}{l}\text { The DL model was built from } \\
\text { 4316 October images ( } 1565 \text { eyes from } \\
\text { patients suffering from glaucoma and } \\
193 \text { normal eyes) used as a pre-training } \\
\text { set. A set of October images trained the } \\
\text { model ( } 94 \text { eyes from patient with early } \\
\text { glaucoma, } 84 \text { healthy eyes). Test set } \\
\text { comprised } 114 \text { eyes from } 114 \text { patients at } \\
\text { early stages of glaucoma and } 82 \text { eyes } \\
\text { from } 82 \text { healthy people }\end{array}$ & $\begin{array}{l}\text { The DL model displayed an area under } \\
\text { the ROC curve of } 93.7 \% \text {, considerably } \\
\text { decreasing (to } 76.6 \text { and } 78.8 \% \text { ) with no } \\
\text { pre-training procedure, suggesting a } \\
\text { relevant sensitivity and specificity of the } \\
\text { DL model to diagnose glaucoma }\end{array}$ & $\begin{array}{l}\text { The use of ML approaches } \\
\text { can offer a significant } \\
\text { improvement in diagnostic } \\
\text { performances, assisting } \\
\text { clinicians in making } \\
\text { a decision }\end{array}$ & [263] \\
\hline $\begin{array}{l}\text { ML-based model } \\
\text { CNN algorithm }\end{array}$ & Diagnostic model for DME & $\begin{array}{c}\text { The model was generated from } \\
\text { 38,057 October images (drusen, CNV, } \\
\text { DME, healthy) by CNN technique. } \\
\text { Training set } 37,457 \text { samples ( } 9891 \mathrm{CNV} \text {, } \\
9633 \text { DME, } 7975 \text { drusen, and } \\
9958 \text { healthy). Validation set } 600 \text { samples } \\
\text { (150 CNV, } 150 \text { DME, } 150 \text { drusen and } \\
150 \text { healthy) }\end{array}$ & $\begin{array}{l}\text { The developed computational tool } \\
\text { showed } 94.5 \% \text { accuracy, } 97.2 \% \text { precision, } \\
97.7 \% \text { sensitivity, and } 97 \% \text { specificity in } \\
\text { the independent testing dataset }\end{array}$ & $\begin{array}{l}\text { October images can be used } \\
\text { for assessing the health of } \\
\text { patients, automatically and } \\
\text { accurately diagnosing several } \\
\text { eye health conditions }\end{array}$ & [258] \\
\hline
\end{tabular}




\subsubsection{AI/ML in Central Nervous System (CNS)-Related Disorders}

Another interesting area in which AI/ML and DL have also been widely employed for brain image assessment to develop imaging-based diagnostic and classification systems is that of neurology and central nervous system (CNS)-related disorders, such as psychiatric disorders, demyelinating diseases, neurodegenerative disorders, epilepsy, and strokes [131,264-269]. Together with extensive usage in image recognition, language processing, and data mining, ML approaches have also obtained growing interest in neurological-related applications, ranging from automated imaging assessment to disorder prediction. In epilepsy, ML approaches are currently applied for automatically detecting seizures using electroencephalography (EEG), video, and kinetic data, automated imaging analysis and pre-surgical planning, prediction of medication response, and prediction of medical and surgical outcomes using several data sources. This was accomplished by different ML techniques, including ANN, SVM, decision tree, RF, and decision forest (Table 6) [269]. For example, in a recent study, Abdelhameed and Bayoumi used EEG data for developing an ML model based on a DL approach for identifying seizures in pediatric patients based on the classification of raw multichannel EEG signal recordings after a limited pre-processing step. The developed ML model based on the CNN technique takes advantage of the automatic feature learning abilities of a two-dimensional deep convolution autoencoder (2D-DCAE) associated with a neural network-based classifier to generate a unified system that is trained in a supervised way to attain the best classification accuracy between the ictal and interictal brain state signals. Generally, two subsequent steps are required for accomplishing the automatic detection of seizure after the acquisition and pre-processing steps of EEG raw signals. The first step involves the extraction and selection of specific characteristics of the EEG signals. In the second stage, it is required to build and train a classification system to use the extracted features for detecting epileptic events. Notably, the step regarding feature extraction directly influences the accuracy/precision of the developed automatic seizure detection model. In the mentioned study, the used dataset was recorded at Boston Children's Hospital, and consists of the long-term EEG scalp recordings of 23 pediatric patients with intractable seizures, while a DL-based system using a supervised 2D-DCAE approach was used for retrieving epileptic seizures in the multichannel EEG signal recording. In order to test and assess the strategy, two models were developed and evaluated, employing three different EEG data segment lengths and a 10-fold cross-validation scheme. Considering five evaluation metrics, the best-performing ML-based tool was a supervised DCAE. In particular, this model showed $98.79 \%$ accuracy, $98.72 \%$ sensitivity, $98.86 \%$ specificity, $98.86 \%$ precision, and an F1-score of $98.79 \%$ [268]. According to this study and other similar research works in the field, the use of ML-based models can be useful in detecting seizures in epilepsy. Furthermore, due to the improvement in processing capabilities, the availability of efficient and more sophisticated ML methods, and the collection of larger datasets, scientists will benefit from these computational approaches, with considerable progress acquired in their use in epilepsy $[268,269]$. 
Table 6. Main examples of AI/ML in CNS-related disorders.

\begin{tabular}{|c|c|c|c|c|c|}
\hline AI Technique & Target & Dataset & $\begin{array}{l}\text { Statistical } \\
\text { Parameters }\end{array}$ & Outcomes & Ref \\
\hline $\begin{array}{l}\text { ML model based on } \\
\text { CNN technique }\end{array}$ & $\begin{array}{l}\text { Automatic detection of seizure } \\
\text { for identifying epileptic events }\end{array}$ & $\begin{array}{l}\text { Extraction and selection of specific } \\
\text { characteristics of the EEG signal dataset was } \\
\text { recorded at Boston Children's Hospital from } \\
23 \text { pediatric patients with intractable seizures } \\
\text { The model was trained using } 117 \text { adults }\end{array}$ & $\begin{array}{l}\text { The model showed accuracy } 98.79 \% \text {, } \\
\text { sensitivity } 98.72 \% \text {, specificity } 98.86 \% \text {, } \\
\text { precision } 98.86 \% \text {, F1-score of } 98.79 \%\end{array}$ & $\begin{array}{l}\text { ML-based models can be useful } \\
\text { in detecting seizure in epilepsy }\end{array}$ & [268] \\
\hline $\begin{array}{l}\text { ML algorithm based on } \\
\text { SVM technique }\end{array}$ & $\begin{array}{c}\text { Classification of adult ADHD } \\
\text { using EEG data }\end{array}$ & $\begin{array}{l}\text { (67 ADHD, } 50 \text { healthy) from four conditions: } \\
\text { two resting conditions (eyes open and eyes } \\
\text { closed) and two neuropsychological tasks } \\
\text { (visual and emotional continuous } \\
\text { performance tests). Four datasets (one for } \\
\text { each condition) independently trained } \\
\text { diverse SVM classifiers }\end{array}$ & $\begin{array}{c}\text { Model performances: } \\
\text { normal vs. ADHD }>70 \% \\
\text { ADHD II vs. ADHD III }>90 \% \\
\text { ADHD III vs. ADHD IV }>87 \%\end{array}$ & $\begin{array}{l}\text { ML-based model discriminated } \\
\text { patients with ADHD from } \\
\text { healthy subjects, differentiating } \\
\text { ADHD subtypes }\end{array}$ & [270] \\
\hline ML-based model & $\begin{array}{l}\text { Prediction of ADHD by } \\
\text { employing CPT indices }\end{array}$ & $\begin{array}{l}\text { CPT indices from } 458 \text { children were used for } \\
\text { training, cross-validating, and testing ML } \\
\text { models (age 6-12 years, } 213 \text { ADHD patients } \\
\text { and } 245 \text { healthy) }\end{array}$ & $\begin{array}{l}\text { The tool was capable of discriminating } \\
\text { patients with ADHD, showing an accuracy of } \\
87 \% \text {, sensitivity of } 89 \% \text {, and specificity of } 84 \%\end{array}$ & $\begin{array}{l}\text { ML models can accurately } \\
\text { classify ADHD using CPT data }\end{array}$ & [271] \\
\hline ML model based on RF technique & $\begin{array}{l}\text { Approach for discriminating } \\
\text { ADHD patients from healthy } \\
\text { subjects using multivariate, } \\
\text { genetic, and PET imaging data }\end{array}$ & $\begin{array}{l}\text { The model was built considering } 16 \text { ADHD } \\
\text { patients and } 22 \text { healthy subjects. These } \\
\text { groups were scanned via PET for measuring } \\
\text { the SERT binding potential. The subjects were } \\
\text { analyzed on the basis of } 30 \text { possible SNPs }\end{array}$ & $\begin{array}{l}\text { The results regarding the model performances } \\
\text { revealed an accuracy of } 0.82 \text {, sensitivity of } \\
0.75 \text {, and specificity of } 0.86\end{array}$ & $\begin{array}{l}\text { The outcomes highlighted the } \\
\text { relevance of SERT along with } \\
\text { SNPs in ADHD, indicating that a } \\
\text { diagnostic tool based on these } \\
\text { features supports clinical } \\
\text { decisions }\end{array}$ & [272] \\
\hline $\begin{array}{l}\text { ML model based on the } \\
\text { CNN technique }\end{array}$ & $\begin{array}{l}\text { Discrimination of ADHD patients } \\
\text { from healthy subjects using data } \\
\text { extracted from EEG analysis }\end{array}$ & $\begin{array}{c}\text { EEG data obtained from } 20 \text { ADHD patients } \\
\text { and } 20 \text { healthy controls were used to train the } \\
\text { model }\end{array}$ & $\begin{array}{c}\text { The computational tool can correctly } \\
\text { categorize ADHD patients with an accuracy } \\
\text { of } 88 \%\end{array}$ & $\begin{array}{l}\text { CNN algorithm built using EEG } \\
\text { data is suitable for developing } \\
\text { diagnostic tools for ADHD }\end{array}$ & [273] \\
\hline $\begin{array}{l}\text { ML-based approach based on DL } \\
\text { technique }\end{array}$ & $\begin{array}{l}\text { Approach for an early diagnosis } \\
\text { of AD from MRI and FDG-PET } \\
\text { images }\end{array}$ & $\begin{array}{l}\text { Data from } 1242 \text { subjects with both a } \\
\text { T1-weighted MRI scan and FDG-PET images } \\
\text { from ADNI database were used for } \\
\text { developing and validating the model. } \\
\text { Subjects were clustered into } 5 \text { classes: (1) sNC } \\
360 \text { subjects; (2) sMCI } 409 \text { subjects; (3) pNC } \\
18 \text { subjects; (4) pMCI } 217 \text { subjects; (5) sAD } \\
238 \text { subjects }\end{array}$ & $\begin{array}{l}\text { The classifier trained using pNC, pMCI, and } \\
\text { sAD samples showed the highest } \\
\text { classification accuracy of } 82.4 \% \text { (identification } \\
\text { of individuals with MCI who will convert to } \\
\text { AD), a } 94.23 \% \text { sensitivity in classifying } \\
\text { persons with probable AD, a } 86.3 \% \text { specificity } \\
\text { in classifying non-dementia controls }\end{array}$ & $\begin{array}{l}\text { The results indicate that DNN } \\
\text { classifiers may be useful as a } \\
\text { potential tool for providing } \\
\text { evidence in support of the } \\
\text { clinical diagnosis of probable AD }\end{array}$ & [274] \\
\hline DL algorithm based on DPN & $\begin{array}{l}\text { Approaches for AD diagnosis } \\
\text { and progression }\end{array}$ & $\begin{array}{c}\text { Data from ADNI dataset (MRI and PET } \\
\text { images from } 51 \text { AD patients, } 99 \mathrm{MCI} \text { patients } \\
\text { (43 MCI-C, who progressed to AD, and } \\
56 \mathrm{MCI}-\mathrm{NC} \text {, who did not progress to AD in } \\
18 \text { months), and } 52 \mathrm{NC}\end{array}$ & $\begin{array}{c}\text { Validation results using ROC curve showed } \\
\text { an area under the curve of } 0.897\end{array}$ & $\begin{array}{l}\text { ML-based approaches for correct } \\
\text { AD diagnosis, classifying all } \\
\text { stages of AD progression }\end{array}$ & [275] \\
\hline
\end{tabular}


Table 6. Cont

\begin{tabular}{|c|c|c|c|c|c|}
\hline AI Technique & Target & Dataset & $\begin{array}{l}\text { Statistical } \\
\text { Parameters }\end{array}$ & Outcomes & Ref \\
\hline ML approach based on CNN & $\begin{array}{l}\text { Classification of CT brain images } \\
\text { for AD patients }\end{array}$ & $\begin{array}{l}\text { Three main groups containing subjects with } \\
\text { AD (1000 images), lesions (e.g., cancer) } \\
\text { (947 images), or normal aging ( } 2129 \text { images). } \\
\text { These data were used for training the model }\end{array}$ & $\begin{array}{c}\text { Accuracy of } 88.8 \%, 76.7 \% \text {, and } 95 \% \text { for groups } \\
\text { of AD, lesion and normal, respectively } \\
\text { (average of } 86.8 \% \text { ) }\end{array}$ & $\begin{array}{l}\text { ML approach based on CNN is } \\
\text { suitable for classifying CT brain } \\
\text { images for AD }\end{array}$ & [276] \\
\hline $\begin{array}{l}\text { ML-based models, } \\
\text { LRCV technique }\end{array}$ & $\begin{array}{c}\text { Extraction of extracting } \\
\text { spectrogram features from } \\
\text { speech data for identifying early } \\
\text { AD }\end{array}$ & $\begin{array}{l}\text { Info from speech dataset, based on the } \\
\text { spectrogram features (extracted based on } \\
\text { audio data using an algorithm ad hoc), that } \\
\text { enclosed AD patients and healthy subjects as } \\
\text { controls. A total of } 36 \text { subjects were included } \\
\text { in the collected speech dataset ( } 23 \mathrm{AD} \\
13 \text { healthy) }\end{array}$ & $\begin{array}{c}\text { LRCV accuracy } 0.833 \text {, precision } 0.869 \text {, recall } \\
0.869, \text { F1-score } 0.869\end{array}$ & $\begin{array}{l}\text { Identification of AD at early } \\
\text { stages for providing therapies for } \\
\text { delaying the disorder progression }\end{array}$ & {$[277]$} \\
\hline $\begin{array}{c}\text { ML approach } \\
\text { EN, SVM, GP, k-NN }\end{array}$ & $\begin{array}{l}\text { Prediction of possible } \\
\text { progression of patients with MCI } \\
\text { and preMCI to AD in } 3 \text { years }\end{array}$ & $\begin{array}{l}\text { ML models were trained employing } \\
\text { information from } 90 \text { patients with MCI and } \\
94 \text { subjects with PreMCI }\end{array}$ & $\begin{array}{l}\text { The best performing ML model based on } \\
\text { SVM technique showed an area under the } \\
\text { ROC curve of } 0.962 \text { and an accuracy of } 0.913\end{array}$ & $\begin{array}{l}\text { Possible use of ML applications } \\
\text { in medical practice and clinical } \\
\text { trials }\end{array}$ & [278] \\
\hline
\end{tabular}

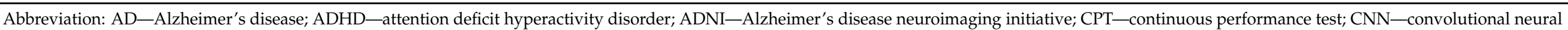

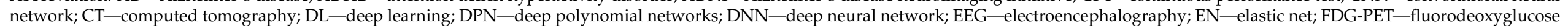

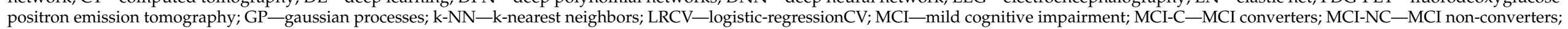

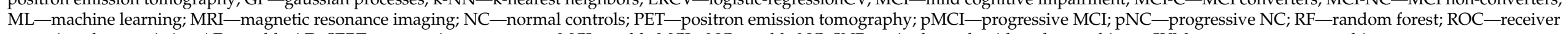
operating characteristic; sAD—stable AD; SERT—serotonin transporter; sMCI—stable MCI; sNC—stable NC; SNPs—single-nucleotide polymorphisms; SVM-support vector machine. 
Considering CNS-related disorders, AI/ML approaches have been used for classifying and diagnosing patients with ADHD (attention deficit hyperactivity disorder). Tenev and colleagues used an ML algorithm based on the SVM technique for classifying adult ADHD using EEG data. The model was trained by enclosing 117 adults (67 ADHD, 50 healthy). Four conditions were considered during measurements: two resting conditions (eyes open and eyes closed) and two neuropsychological tasks (visual continuous performance test and emotional continuous performance test). The authors considered four datasets (one for each condition) that independently trained diverse SVM classifiers. The output was combined, employing a logical expression obtained from the Karnaugh map. The evaluation of the developed computational protocol indicated that following this strategy, it is possible to discriminate patients with ADHD from healthy subjects, differentiating ADHD subtypes [270]. Slobodin and coworkers applied an ML-based model for predicting ADHD by employing a continuous performance test (CPT) index. These data from 458 children were used for training, cross-validating, and testing ML models (age 6-12 years, 213 ADHD patients and 245 healthy). Authors used the CPT total score containing four indices (timeliness, attention, impulsiveness, and hyperactivity) and four variables (gender, age, day of the week, and time of day), to obtain relevant data capable of discriminating patients with ADHD. The developed model showed significant predictivity, displaying accuracy, sensitivity, and specificity of $87 \%, 89 \%$, and $84 \%$, respectively. Interestingly, ML models can accurately classify ADHD using CPT data [271]. In another impressive work, Kautzky and collaborators described the development of an ML model for discriminating ADHD patients form healthy subjects using multivariate, genetic, and positron emission tomography (PET) imaging data. They selected 16 patients with ADHD and 22 healthy subjects. These groups were scanned via PET for measuring the serotonin transporter (SERT) binding potential, employing the radioligand $\left[{ }^{11} \mathrm{C}\right] \mathrm{DASB}$ (3-amino4-(2-dimethylaminomethylphenylsulfanyl)-benzonitrile). The considered subjects were analyzed based on 30 possible single-nucleotide polymorphisms (SNPs) involving HTR1A, HTR1B, HTR2A, and TPH2 genes. Accordingly, authors defined cortical and subcortical regions of interest (ROI), and an ML model based on the RF technique was employed for selecting and classifying relevant features in a 5-fold cross-validation model (10 repeats). The results regarding the model performances revealed an accuracy, sensitivity, and specificity of $0.82,0.75$, and 0.86 , respectively, indicating the significant predictivity of the model. Furthermore, the outcomes highlighted the relevance of SERT along with HTR $1 B$ and HTR2A genes in ADHD, indicating disease-specific effects and suggesting that a diagnostic tool based on these features can be suitable for supporting clinical decisions [272]. In the last example, Dubreuil-Vall and colleagues developed an ML model based on the $\mathrm{CNN}$ technique with a four-layer architecture combining filtering and pooling, employing various types of data extracted from EEG analysis for discriminating ADHD patients from healthy subjects. These data obtained from 20 ADHD patients and 20 healthy controls were used to train the model. Based on the results presented by the authors, the computational tool can correctly categorize ADHD patients, showing an accuracy value of $88 \%$, outperforming other models such as RNN and other ML models previously reported. Although the data are interesting and promising, studies considering a more consistent number of participants are highly desirable [273].

A different field in which the imaging techniques can be helpful during diagnosis is the area of neurodegenerative diseases. In fact, the multifactorial and complex molecular mechanisms involved in neurodegeneration make the discovery of tools for early diagnosis challenging, as well as the identification of effective treatments. In this scenario, ML-based approaches allow this gap to be reduced, assisting researchers in devising an early diagnosis, interpreting brain images and developing potential effective therapeutic strategies [266]. In fact, regarding $\mathrm{AD}$, a precise diagnosis, and its early-stage characterization, such as mild cognitive impairment (MCI), is essential to opportunely treat and possibly slow down $\mathrm{AD}$ progression. Accordingly, Lu and coworkers described an ML-based approach based on the DL technique for an early diagnosis of AD. They proposed a multimodal and multiscale 
ML-based method in which information from magnetic resonance imaging (MRI) and fluorodeoxyglucose positron emission tomography (FDG-PET) images were combined within the DNN framework for discriminating AD patients. For developing the abovementioned model, the following two steps are required: (I) Pre-processing images from MRI and FDG-PET. This step allows the sub-division of the gray matter into patches of a range of sizes, for extracting features from each patch size; (II) Training a DNN algorithm for learning the patterns for discriminating AD individuals. Next, the ML-based model can be employed for an individual classification. Data from 1242 subjects with both a T1-weighted MRI scan and FDG-PET images from the Alzheimer's Disease Neuroimaging Initiative (ADNI) database were used for developing and validating the model. Subjects were clustered into five classes based on clinical diagnosis: (1) stable normal controls (sNC), 360 subjects; (2) stable MCI (sMCI), 409 subjects; (3) progressive NC (pNC), 18 subjects assessed to be NC at baseline visit but progressed to a clinical diagnosis of possible AD; (4) progressive MCI (pMCI), 217 subjects evaluated to be $\mathrm{MCI}$ at the baseline visit and progressed to a clinical diagnosis of possible AD at some point in the future; and (5) stable $\mathrm{AD}$ (sAD), 238 subjects with AD. Furthermore, the classifier trained with the combined samples of $\mathrm{pNC}, \mathrm{pMCI}$, and $\mathrm{sAD}$ was found to yield the highest overall classification accuracy of $82.4 \%$ (accuracy in identifying individuals with MCI who will convert to AD at 3 years before conversion ( $86.4 \%$ combined accuracy for conversion within $1-3$ years)), a $94.23 \%$ sensitivity in the classification of persons with a clinical diagnosis of probable $\mathrm{AD}$, and an $86.3 \%$ specificity in the classification of non-dementia controls. These results suggest that DNN classifiers may be useful as a potential tool for providing evidence in support of the clinical diagnosis of probable AD [274]. Shi and colleagues highlighted the importance of combining information derived from different tests. To this end, they developed a DL algorithm based on deep polynomial networks (DPN) to develop a computational model trained by multimodal neuroimaging data (MRI and PET). In the selected work, they built a multimodal stacked DPN (MM-SDPN) algorithm. MM-SDPN involves two SDPN stages, one dedicated to fusing multimodal neuroimaging data, and the other devoted to learning high-level features from AD diagnosis. The authors used data from the ADNI dataset (same MRI and PET images from $51 \mathrm{AD}$ patients, $99 \mathrm{MCI}$ patients (43 MCI converters [MCI-C], who progressed to $\mathrm{AD}$, and $56 \mathrm{MCI}$ non-converters [MCI-NC], who did not progress to $\mathrm{AD}$ in 18 months), and 52 normal controls (NC)). The developed MMSDPN algorithm was applied to the ADNI dataset for conducting both binary classification and multiclass classification tasks. Validation results using a ROC curve showed an area under the curve of 0.897 , indicating that the MM-SDPN approach performed better than other multimodal ML-based approaches in achieving correct AD diagnosis, being able to classify all stages concerning AD progression [275]. Gao and collaborators, by using an ML approach based on the CNN technique, classified computed tomography (CT) brain images with the aim to translate images into clinical applications. This classification was carried out considering three main groups: containing subjects with AD (1000 images), lesions (e.g., cancer) (947 images), or normal aging (2129 images). Interestingly, because of the features of CT brain images with higher thickness, the authors considered both 2D and $3 \mathrm{D} \mathrm{CNN}$ in this research. The fusion was consequently performed considering both 2D CT images along the axial direction and 3D segmented blocks with accuracy rates of $88.8 \%$, $76.7 \%$, and $95 \%$ for groups of $\mathrm{AD}$, lesion, and normal, respectively, leading to an average of $86.8 \%$. Accordingly, adopting the ML approach based on CNN makes it possible to classify CT brain images for AD with great accuracy [276]. In another interesting approach, Liu and collaborators conceived a different ML approach to identify AD. In particular, the authors collected a novel speech dataset, based on the spectrogram features (extracted based on audio data using an algorithm ad hoc) that enclosed AD patients and healthy subjects as a control. Next, ML-based models were employed for comparing this new dataset with the speech provided by the Dem@Care project. Among the assessed ML-based models, the logistic regression CV (LRCV) model showed the best performance. Notably, the authors demonstrated that ML-based approaches, trained by extracting spectrogram features from 
speech data, can be applied for identifying $\mathrm{AD}$, helping in understanding the development of $\mathrm{AD}$ at early stages for providing therapies to delay disorder progression [277]. Finally, we report an interesting ML approach described by Grassi and colleagues. Their study focused on the development of an algorithm for predicting, based on a time of 3 years, a possible progression of patients with $\mathrm{MCI}$ and preMCI to $\mathrm{AD}$. ML models were trained by employing information from 90 patients with MCI and 94 subjects with PreMCI, with a diagnostic follow-up evaluation for at least 3 years. They extracted several features from the data for a total of 36 predictors (e.g., diagnostic subtypes, clinical and neuropsychological test scores, sociodemographic characteristics, cardiovascular risk indexes, and levels of medial temporal lobe brain atrophy in the hippocampus (HPC), the perirhinal cortex (PRC), and the entorhinal cortex (ERC), and assessed by a clinician-rated Visual Rating Scale (VRS)). To model these data, the authors used several ML-based techniques, including Elastic Net (EN) with polynomial features, SVM, Gaussian processes (GP), and $\mathrm{k}-\mathrm{NN}$. The resulting models were validated using leave-pair-out-cross-validation. The best-performing ML model based on the SVM technique showed an area under the ROC curve of 0.962 , with an accuracy of 0.913 [278]. The reported work further demonstrated how ML applications can assist translational research, providing computational tools for prompt applications in medical practice and clinical trials. Similarly, comparable procedures, extracting specific features from available data, allowing the development of ML-based models for Parkinson's disease (PD). In fact, as reported for AD, several studies highlighted that through ML-based approaches applied to PD [279], it is possible to predict the progression of the disorder by employing serum cytokines [280], MRI [281], and walking tests [282]; to estimate the state of PD, employing longitudinal data [283]; to rate the main symptoms (resting tremor and bradykinesia) [284]; and to produce a correct diagnosis from EEG analysis $[285,286]$ and from voice datasets $[287,288]$.

\subsubsection{AI in Cardiology and Cardiovascular Diseases}

Due to the enormous progress in cardiovascular imaging, along with the advancement of recording technologies, enabling the acquisition of complex and huge multi-dimensional data, AI/ML can be applied in cardiology. ML-based techniques allow cardiologists to investigate new possibilities, producing findings not detected using classical strategies. Additionally, considering this field, ML can offer novel chances for improving patient support (survival prediction, appropriate diagnoses, and pharmacological treatments) and medical decision making, covering the gap between the swift progress of cardiac imaging and clinical care $[134,289,290]$. Several studies in cardiology and related fields employed supervised ML models as diagnostic predictors [291,292]. These computer-based tools can extract specific features obtained from imaging data and clinical outcomes, selecting features derived from any imaging data sample (e.g., electrocardiograms (ECG), echocardiograms, cardiac MRI, cardiac computed tomography (CCT)) for providing specific diagnoses [293]. In this section, some relevant and innovative examples of ML applications in cardiology field are examined and discussed (Table 7). Madani and colleagues developed an ML protocol based on the DL approach using the CNN algorithm for establishing an $\mathrm{AI}$ tool to interpret echocardiograms. They trained a $\mathrm{CNN}$ using images and video from 267 transthoracic echocardiograms depicting real-world clinical variation (e.g., different patient variables, echocardiographic indications, technical qualities, and pathologies) for classifying 15 distinct standard echocardiographic views. For generating the CNN model, they employed over 200,000 images (240 studies) for arranging a training and validation set of over 20,000 images (27 studies), comprising the test set. The developed computer-based model showed an overall accuracy of $97.8 \%$ on videos ( $F$-score 0.964 ) and of $100 \%$ on seven of the twelve video views, supporting the robustness of the approach [294]. Another study performed by Madani and colleagues reported the development of an ML-based approach using the $\mathrm{CNN}$ technique, employing DL classifiers for automatically interpreting echocardiographic data. The results from this report showed an accuracy of $94.4 \%$, considering 15 echocardiographic view classifications of still images and 91.2\% accuracy for binary 
left ventricular hypertrophy view classification. Subsequently, the authors employed a semi-supervised generative adversarial network model for detecting left ventricular hypertrophy. The model showed excellent performances, accounting for an accuracy of $80 \%$ in view classification and of $92.3 \%$ accuracy for left ventricular hypertrophy [295]. Zhang and colleagues reported the development of different ML models based on the CNN technique for an automatic classification of echocardiogram data for detecting three distinct cardiovascular diseases: hypertrophic cardiomyopathy, cardiac amyloid, and pulmonary arterial hypertension. For training and validating the models for multiple tasks, the authors used 14,035 echocardiograms, spanning a 10-year period. The results were assessed by comparing data from manual segmentation and measurements considering 8666 echocardiograms from routinary clinical assessment. The developed CNN models were able to identify views, including flagging partially obscured cardiac chambers and facilitated the segmentation of individual cardiac chambers. Overall, the authors' findings demonstrated that automated measurements can be similar or even superior to manual measurements, considering 11 internal consistency metrics (e.g., the correlation of left atrial and ventricular volumes). Furthermore, CNN models appropriately detect hypertrophic cardiomyopathy, cardiac amyloidosis, and pulmonary arterial hypertension, showing $C$ statistical parameters of $0.93,0.87$, and 0.85 , respectively [296]. Interestingly, echocardiography outcomes were used from Samad and colleagues to develop a supervised ML model based on RF algorithm to predict future adverse cardiac events. In fact, the RF algorithm was employed for predicting survival from echocardiography data. They trained the model employing the information obtained from echocardiograms considering 171,510 patients, providing three different classes of input: (I) clinical variables, such as 90 cardiovascular-relevant international classification of diseases (ICD)-10 codes, sex, weight, age, height, blood pressures, heart rate, LDL, HDL, and smoking; (II) clinical variables plus physician-reported ejection fraction; and (III) clinical variables, ejection fraction, plus 57 additional echocardiographic measurements. The ML models based on the RF algorithm showed good accuracy regarding the prediction, with an area under the ROC curve $>0.82$ greater than conventional clinical risk scores (area under the ROC curve ranging from 0.61 to 0.79). Accordingly, ML can successfully use combining several and distinct input variables for predicting survival considering echocardiography data [297]. Again, the CNN technique was also used from Strodthoff and coworkers for developing an ML model for detecting myocardial infarction directly from ECG with no preprocessing. They used a dataset of 549 ECG outcomes from 290 subjects available from the Physikalisch Technische Bundesanstalt (PTB) database, which enclosed a large amount of publicly accessible ECG data. The developed ML model based on a DL approach showed a sensitivity and specificity of $93.3 \%$ and $89.7 \%$, respectively, as assessed by employing a 10-fold cross-validation with sampling established on patients. The described model was able to detect myocardial infarction and it showed performances comparable with those obtained from human cardiologists. Furthermore, another analysis showed that it is also able to discriminate channel-specific regions, substantially contributing to the neural network's decision. This highlighted that the same signs indicative of myocardial infarction recognized by human cardiologists were underlined from the ML model. This work further demonstrated that ML models applied to ECG evaluation can be progressed into clinical application [298]. Hannun and coworkers developed an ML model based on the DNN technique, employing ECG data, for detecting arrhythmias. The DNN algorithm was trained using 91,232 single-lead ECG records from 53,549 patients who used a single-lead ambulatory ECG monitoring device for classifying 12 rhythm classes (10 arrhythmias, as well as sinus rhythm and noise). The resulting model was validated using an independent test set (328 ECGs collected from 328 patients), showing an average area under the ROC curve of 0.97 . Moreover, the median $F_{1}$ score, which represents the harmonic mean of the positive predictive value and sensitivity, for the DNN (0.837) surpassed that of average cardiologists (0.780) for all rhythm classes. The results clearly indicate that the ML approach based on DNN can be used for correctly classifying different types of arrhythmias from ECG outcomes. This approach could hold 
tremendous potential if used in clinical settings, reducing misdiagnoses to prioritize urgent health status [299]. Recently, Elul and colleagues used ECG data for developing an ML model to detect a heterogeneous combination of known and unknown arrhythmias and to identify underlying cardio-pathology, considering segments marked as normal sinus rhythm documented in patients with intermittent arrhythmia [300]. Furthermore, asymptomatic left ventricular dysfunction (ALVD) can be predicted using a CNN algorithm employing ECG data, as reported by Attia and colleagues. The authors used paired 12-lead ECG and echocardiogram data, including the left ventricular ejection fraction (a measure of contractile function), considering 44,959 patients for training a CNN algorithm for identifying subjects affected by ventricular dysfunction (defined as ejection fraction $\leq 35 \%$ ). The developed model was tested against an independent set of 52,870 subjects, showing an area under the ROC curve, accuracy, specificity, and sensitivity of $0.93,85.7 \%, 85.7 \%$, and $86.3 \%$, respectively. Very interesting is that the authors found that in patients devoid of ventricular dysfunction, those with positive outcomes, indicated by the ML model, were at four times the risk (hazard ratio, 4.1; 95\% confidence interval, 3.3 to 5.0) of developing future ventricular dysfunction compared with those with a negative screen. Remarkably, the application of AI/ML to ECG data is versatile for predicting many possible outputs, in order to find potential subjects who will develop a given disorder, as in the case of ALVD [301]. The following example reported the use of an unsupervised ML approach for assessing diastolic dysfunction. The objective of the study conducted by Pandey and collaborators was to develop an ML model based on the DNN technique for integrating multidimensional echocardiographic data, with the aim to detect distinct patient subgroups with heart failure in conjunction with preserved ejection fraction (HFpEF). This study is particularly relevant, since, currently, no algorithms translated for clinical use exist for phenotyping the severity of diastolic dysfunction in HFpEF. The authors established a DNN model for predicting high- and low-risk phenogroups in a derivation group $(n=1242)$. Next, two external groups were considered for validating the performance of the model in identifying high left ventricular filling pressure $(n=84)$ and assessing its prognostic capacity in patients $(n=219)$ presenting different degrees of systolic and diastolic dysfunction. Notably, the clinical relevance of the ML model was evaluated in three HFpEF clinical trials by assessing the relationships of the groups with adverse clinical consequences (TOPCAT trial, NCT00094302, $n=518$ ), cardiac biomarkers, and exercise parameters (NEAT-HFpEF trial, NCT02053493, and RELAX trial, $n=346)$. Notably, the developed unsupervised ML model based on the DNN technique showed an area under the ROC curve higher than that reported by the American Society of Echocardiography guidelines for predicting high left ventricular filling pressure ( 0.88 vs. $0.67 ; p=0.01)$. Furthermore, the developed model showed high performance when also considering the validation sets, including the three HFpEF clinical trials. In fact, the DNN classifier can depict the severity of diastolic dysfunction and identify a specific subgroup of patients with HFpEF showing high left ventricular filling pressure, biomarkers of myocardial injury and stress, and adverse events, and those who are more likely to respond to spironolactone [302]. Another interesting application of an ML model applied to the cardiovascular system was described by Ma and coworkers. They started considering the relationships between carotid plaque echogenicity in ultrasound images and the risk of stroke in atherosclerotic patients. For accurately classifying carotid plaques to estimate their stability to predict cardiovascular events, the authors used an ML model employing the CNN technique. This approach automatically provides a carotid plaque echogenicity classification. For improving the reliability of the method, the authors redesigned the spatial pyramid pooling (SPP) and proposed multilevel strip pooling (MSP) for the automatic and accurate classification of carotid plaque echogenicity in the longitudinal section. By performing this step, the resulting MPS module was able to accept arbitrarily sized carotid plaques as input and capture a long-range informative context for improving the accuracy of classification. Accordingly, the scientists implemented an MSP-based CNN, employing the visual geometry group (VGG) network as the backbone. They trained the model using 1463 carotid plaque images (335 echo-rich plaques, 405 inter- 
mediate plaques, and 723 echolucent plaques). The five-fold cross-validation results show that the proposed MSP-based VGGNet achieved a sensitivity of $92.1 \%$, specificity of $95.6 \%$, accuracy of $92.1 \%$, and F1-score of $92.1 \%$. The findings of this work prove that this strategy is relevant for enhancing the applicability of $\mathrm{CNN}$ using any input size of samples, leading to an improvement in the accuracy of classification, making the objective risk assessment more effective [303].

The rising usage of ML-based approaches in cardiology is likely to continue in the foreseeable future. Following a proper validation, they might enhance treatment outcomes by facilitating daily workflow, patient satisfaction, early identification, and the correct interpretation of data. 
Table 7. Main examples of AI/ML in cardiology and cardiovascular diseases.

AI Technique Target

Dataset

Statistical Parameters

The model was trained using images and video (267 transthoracic echocardiograms) consisting of 200,000 images (240 studies) for

DL approach CNN algorithm

I tool to interpre

echocardiograms

Development of DL

ML-based approach using CNN technique

ML models based on $\mathrm{CNN}$ technique

Supervised ML model based on RF algorithm

ML model based on $\mathrm{CNN}$ technique

ML model based on DNN technique interpreting

echocardiography data

Approach for an automatic classification of

echocardiograms to detect three cardiovascular diseases: hypertrophic cardiomyopathy, cardiac amyloid, and pulmonary arterial hypertension Approach for predicting future adverse cardiac events-RF algorithm for predicting survival from echocardiography data

ML model for detecting myocardial infarction directly from ECG with no preprocessing

Approach for detecting arrhythmias employing ECG data classifiers for automatically rranging a training and validation set and a test set of over 20,000 images

The model was built using a dataset of 347,726 echocardiogram images $(325,980$ images were in the training set)

For training and validating the models 14,035 echocardiograms spanning a

10-year period were used. Results were assessed by comparing data from measurements considering 8666 echocardiograms from clinical assessment

The model was trained using echocardiograms from 171,510 patients

Dataset of 549 ECG outcomes from 290 subjects (PTB database) was used

The model was trained using 91,232 single-lead ECG records from 53,549 patients for classifying 12 rhythm classes (10 arrhythmias, sinus rhythm and noise). Validation test set 328 ECGs collected from 328 patients manual segmentation and
The developed computer-based model showed an overall accuracy of $97.8 \%$ on videos ( $F$-score 0.964$)$ and of $100 \%$ on seven of the 12 video view

The model showed accuracy of $94.4 \%$ considering 15 echocardiographic view classifications of still images and 91.2\% accuracy for binary left ventricular hypertrophy view classification

CNN models appropriately detect hypertrophic cardiomyopathy, cardiac amyloidosis, and pulmonary arterial hypertension showing $C$ statistical parameters of $0.93,0.87$, and 0.85 , respectively

The ML model showed an area under the ROC curve $>0.82$ greater than conventional clinical risk scores (area under the ROC curve ranging from 0.61 to 0.79 )

The ML model showed sensitivity of $93.3 \%$ and specificity of $89.7 \%$ as assessed employing 10-fold cross-validation with sampling established on patients

The ML model showed an area under the ROC curve of 0.97 . The $F_{1}$ score of 0.837 surpassed that of average cardiologists (0.780) for all rhythm classes
Outcomes

Ref

The use of CNN algorithms is suitable for a correct interpretation of echocardiograms

Efficient DL solutions for medical imaging assessment in cardiology

ML models are useful for classifying echocardiograms and for detecting cardiovascular disorders

ML can successfully be used for predicting survival considering echocardiography data

The model detected myocardial infarction with performances comparable with those obtained from human cardiologists

The results clearly indicate that the ML approach based on DNN can be used for correctly classifying different types of arrhythmias from ECG outcomes 
Table 7. Cont

\begin{tabular}{|c|c|c|c|c|c|}
\hline AI Technique & Target & Dataset & $\begin{array}{l}\text { Statistical } \\
\text { Parameters }\end{array}$ & Outcomes & Ref \\
\hline $\mathrm{CNN}$ algorithm & $\begin{array}{l}\text { ALVD can be predicted } \\
\text { employing ECG data }\end{array}$ & $\begin{array}{c}\text { Dataset composed of } \\
\text { ECG/echocardiogram data from } \\
44,959 \text { patients for training a CNN } \\
\text { algorithm. The developed model was } \\
\text { tested against an independent set of } \\
52,870 \text { subjects }\end{array}$ & $\begin{array}{c}\text { The model showed an area under the } \\
\text { ROC curve, accuracy, specificity, and } \\
\text { sensitivity of } 0.93,85.7 \%, 85.7 \% \text {, and } \\
86.3 \% \text {, respectively }\end{array}$ & $\begin{array}{l}\text { AI/ML to ECG data is } \\
\text { versatile for predicting } \\
\text { possible outputs for finding } \\
\text { potential subjects who will } \\
\text { develop ALVD }\end{array}$ & [301] \\
\hline $\begin{array}{l}\text { Unsupervised ML approach } \\
\text { based on DNN technique }\end{array}$ & $\begin{array}{l}\text { Approach for assessing } \\
\text { diastolic dysfunction } \\
\text { integrating multidimensional } \\
\text { echocardiographic data with } \\
\text { the aim to detect distinct } \\
\text { patient subgroups } \\
\text { with HFpEF }\end{array}$ & $\begin{array}{l}\text { The established DNN model predicted } \\
\text { high- and low-risk phenogroups in a } \\
\text { derivation group }(n=1242) \text {. Two } \\
\text { external groups for validating the model } \\
\text { to identify high left ventricular filling } \\
\text { pressure }(n=84) \text { and assessing its } \\
\text { prognostic capacity in patients }(n=219) \\
\text { showing different degrees of systolic and } \\
\text { diastolic dysfunction }\end{array}$ & $\begin{array}{l}\text { The relevance of the ML model was } \\
\text { evaluated in three HFpEF clinical trials } \\
\text { by assessing the relationships of the } \\
\text { groups with adverse clinical outcomes. } \\
\text { The developed model showed an area } \\
\text { under ROC curve higher than that } \\
\text { reported by the American Society of } \\
\text { Echocardiography guidelines for } \\
\text { predicting high left ventricular filling } \\
\text { pressure }(0.88 \text { vs. } 0.67 ; p=0.01)\end{array}$ & $\begin{array}{l}\text { The DNN classifier can depict } \\
\text { the severity of diastolic } \\
\text { dysfunction and identify a } \\
\text { specific subgroup of patients } \\
\text { with HFpEF }\end{array}$ & [302] \\
\hline $\begin{array}{l}\text { ML model employing } \\
\text { CNN technique }\end{array}$ & $\begin{array}{l}\text { Approach for accurately } \\
\text { classifying carotid plaques to } \\
\text { estimate their stability to } \\
\text { predict cardiovascular events }\end{array}$ & $\begin{array}{l}\text { The model was trained using } \\
1463 \text { carotid plaque images ( } 335 \\
\text { echo-rich plaques, } 405 \text { intermediate } \\
\text { plaques, and } 723 \text { echolucent plaques) }\end{array}$ & $\begin{array}{l}\text { The model showed sensitivity of } 92.1 \% \text {, } \\
\text { specificity of } 95.6 \% \text {, accuracy of } 92.1 \% \text {, } \\
\text { F1-score of } 92.1 \%\end{array}$ & $\begin{array}{l}\text { The findings of this work } \\
\text { proved that this strategy is } \\
\text { relevant for enhancing the } \\
\text { applicability of CNN using } \\
\text { any input size of samples }\end{array}$ & [303] \\
\hline
\end{tabular}

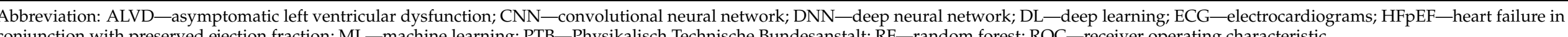
conjunction with preserved ejection fraction; ML—machine learning; PTB—Physikalisch Technische Bundesanstalt; RF—random forest; ROC—receiver operating characteristic. 


\subsection{6. $\mathrm{AI}$ in Gastroenterology}

In the area of gastroenterology, clinicians work with many clinical data and several imaging technologies, including endoscopy and ultrasound. In this context, for managing and analyzing huge quantities of information, AI/ML methodologies can play a pivotal role regarding image analysis, diagnosis, prognosis, and possible treatments. AI/ML-based techniques can be applied to gastroenterology for improving endoscopic diagnosis, allowing the detection of abnormalities of the gastrointestinal tract such as colorectal polyps, malignancies such as esophageal, gastric, and intestinal tumors, and conditions such as inflammatory bowel disease, irritable bowel syndrome, and peptic ulcer bleeding [304-306]. We report here some relevant examples demonstrating the translational potential of the AI/ML-based approach in gastroenterology (Table 8). Mori and collaborators reported an AI approach for detecting small $(<5 \mathrm{~mm})$ adenomatous or sessile polyps, usually extremely difficult to identify for clinicians employing colonoscopy. For validating the approach in a prospective, single-group, and open-label clinical trial (UMIN000027360), they trained an ML-based model with data from 325 subjects presenting 466 microscopic polyps. In this prospective study, the model showed an accuracy of $94 \%$ (with a negative predictive value of $96 \%$ ), including a pathologic prediction rate of $98.1 \%$ (457 of 466) [307]. In another approach, Wang and colleagues developed an ML algorithm for detecting polyps in clinical colonoscopy investigations. Specifically, they generated a DL algorithm trained by employing data derived from 1290 patients (5545 colonoscopy images). The training of the model was performed in two separate steps: (1) A training step in which 4495 images were used, selecting 2607 images containing polyps and 1888 images with no polyps. The training data were employed for optimizing the network parameters; (2) A tuning step in which 1050 images (1027 with polyps and 23 without polyps) were considered for optimizing hyperparameters. The authors validated the approach using information obtained from (I) A novel collected set consisting of 27,113 colonoscopy images taken from 1138 patients, presenting, as a minimum, one detected polyp. The calculated statistical parameters demonstrated the validity of the approach, showing a sensitivity of $94.38 \%$ and a specificity of $95.92 \%$, with an area under the ROC curve of 0.984; (II) A public database containing clinical images of 612 polyps (sensitivity of $88.24 \%$ ); (III) A total of 138 colonoscopy videos, including histologically established polyps (sensitivity of $91.64 \%$; per-polyp sensitivity of $100 \%$ ); (IV) A set of 54 intact full-range colonoscopy videos with no polyps (specificity of $95.40 \%$ ). The developed DL model has great potential in assisting clinicians while conducting colonoscopy, being able to correctly discriminate polyps and adenomas [308]. Byrne and coworkers developed an ML model based on the deep CNN technique for real-time evaluation of endoscopic video images of colorectal polyps. The model was trained and validated using untouched video data derived from routine clinical investigations not adapted for classification based on an $\mathrm{AI}$ approach. For assessing the performance of the developed computational tool, the authors tested the model, employing an independent set of 125 videos of sequentially encountered diminutive polyps classified as adenomatous or hyperplastic polyps. The ML model showed a sensitivity of $98 \%$, a specificity of $83 \%$, and an accuracy of $94 \%$, being able to discriminate hyperplastic from adenomatous polyps [309]. Urban and colleagues used a similar approach to develop a deep CNN algorithm for detecting polyps from colonoscopy exams. They trained an ML model by employing 8641 hand-labeled images, with 4088 unique polyps, from colonoscopy screenings derived from over 2000 subjects. The authors tested the model using 20 colonoscopy videos ( $5 \mathrm{~h}$ of duration). When validated considering manually labeled images, the developed model detected polyps with an area under the ROC curve of 0.991 and an accuracy of $96.4 \%$. Interestingly, in the examination of colonoscopy videos where 28 polyps were removed, four expert reviewers found eight extra polyps with no ML-based support that had not been removed and observed further 17 polyps by taking advantage of CNN support (45 total polyps). Notably, every one of the polyps removed and detected by experts were found using the ML-based model, although the computational tool showed $7 \%$ false positives. However, the CNN algorithm identified a number of polyps higher than that observed by 
expert clinicians. Notably, the additional polyps found by the model were small adenomas with a size ranging from 1-3 to 4-6 mm [310]. Regarding gastrointestinal malignancies, some methods, based on $\mathrm{AI} / \mathrm{ML}$, for detecting cancers in the gastrointestinal tract have been described. For example, Tokai and colleagues, in their study, estimated the diagnostic capability of an ML tool based on the CNN algorithm in detecting esophageal squamous cell carcinoma (ESCC) and assessing its invasiveness. For a comprehensive assessment of the performances, they compared the acquired results with the findings obtained from thirteen expert endoscopists. The $\mathrm{CNN}$ algorithm was trained using white light imaging and narrow-band imaging endoscopic images, including 1751 images of ESCC. In the validation step, the ML-based model identified $95.5 \%$ of ESCC in test pictures $(279 / 291)$ in ten seconds, properly estimating the invasion depth of ESCC, with a sensitivity of $84.1 \%$ and accuracy of $80.9 \%$, in six seconds. The diagnosis assisted by the CNN algorithm was more accurate than the diagnosis of expert clinicians alone, indicating a potential role of ML as an ESCC diagnostic tool [311]. Another example of an AI/ML application to detect cancer and its invasive potential was carried out by Nakagawa and collaborators. They reported the development of a DNN approach for diagnosing the invasion depth of ESCC. The ML-based model was built by employing endoscopic images from subjects affected by superficial ESCC. In particular, the authors generated a training set by collecting 8660 non-magnified endoscopic images, as well as 5678 magnified images, from 804 patients with superficial ESCC presenting cancer invasion, while they compiled a validation test set consisting of 405 non-magnified images and 509 magnified images from 155 subjects. The DNN algorithm showed the following statistical parameters: specificity $95.8 \%$, sensitivity $90.1 \%$, accuracy $91 \%$, positive predicted value $99.2 \%$, and negative predictive value $63.9 \%$. These parameters highlighted the capacity of the model to identify pathologic mucosal and submucosal microinvasive (SM1) cancers from submucosal deep invasive (SM2/3) cancers. Compared with the assessment performed by a pool of experts, employing the same validation set, the model showed a slight improvement in the performances, confirming the ability to detect the invasion depth in patients with superficial ESCC [312]. Other interesting works in the field regard the possibility to assess the severity of inflammatory bowel disease (IBD) and improve its classification by using the AI/ML approach. Ozawa and coworkers developed an ML-based system for evaluating the severity of ulcerative colitis. They developed a CNN algorithm trained on colonoscopy images (26,304 images) derived from 841 subjects affected by ulcerative colitis. The performance of the ML model was assessed considering an independent test set composed of 3981 images from 114 patients with ulcerative colitis. The model was examined for its capacity to distinguish normal mucosa (Mayo 0) and mucosal healing state (Mayo 0-1). The validation was achieved by calculating the areas under the ROC curve, and the results for the ML-based model were 0.86 and 0.98 in identifying Mayo 0 and $0-1$, respectively. The CNN algorithm better performed for the rectum than for the right side and left side of the colon when identifying Mayo 0 (areas under the ROC curve $=0.92,0.83$, and 0.83 , respectively). This work underlined the robustness of the method in identifying endoscopic inflammation seriousness in subjects with ulcerative colitis, indicating that the $\mathrm{CNN}$ algorithm can assist clinicians in determining severity-based therapies as well as follow-up endoscopy waits for IBD [313]. Mossotto and collaborators developed an ML model for classifying pediatric IBD, employing data derived from endoscopic and histological imaging of 287 children affected by IBD. These data were used for developing, training, testing, and validating an ML model for classifying disorder subtypes. Unsupervised ML models displayed wide clustering of Crohn's disease/ulcerative colitis, but no apparent subtype differentiation, while hierarchical clustering recognized new categories with varying levels of colonic contribution. Furthermore, endoscopic data alone, histological data alone, and a combination of endoscopic/histological data were used to generate three supervised ML models, showing a classification accuracy of $71.0 \%, 76.9 \%$, and $82.7 \%$, respectively. The most promising ML model was assessed by considering an independent group of 48 children affected by IBD. The findings demonstrated that the ML-based model appropriately classified patients 
with an accuracy of $83.3 \%$. This work highlighted that for the development of a proper supervised ML model, it is necessary to consider both endoscopic and histological data for performing a more accurate classification of a disease [314].

A fascinating approach in which the AI/ML-based approach can be used is in the field of food intolerance. In particular, starting from a decade ago, several computational attempts were made for detecting subjects presenting celiac disease and for classifying the disorder [315]. In a pioneering approach, Vècsei and collaborators developed a computerbased methodology for automatically classifying celiac severity on 612 endoscopic images from pediatric patients considering a two-class issue: mucosa affected by celiac disease and unaffected duodenal tissue. Even though the classification method was able to discriminate celiac disease into two groups (disease vs. no disease), showing an overall accuracy of $88 \%$, the model displayed a reduced accuracy $(63.7 \%)$ in classifying the severity of disorders, possibly due to the small set for training the model [316]. Afterwards, Wimmer and collaborators theorized that AI methods can be employed for classifying luminal endoscopic images of celiac disease. They developed a CNN transfer-learning method that categorized luminal endoscopic images from the duodenum gathered by white light and narrow-band imaging endoscopy, collecting 1661 images. The CNN algorithm showed an accuracy of $90.5 \%$ in the identification of celiac disease considering endoscopic images alone. The authors indicated that while the gold standard for the diagnosis of celiac disease remains unchanged, ML could offer a new method in diagnostic settings, especially where acquiring biopsies is complicated [317]. Hujoel and collaborators developed an ML model for detecting undiagnosed celiac disease. For this purpose, they collected serum samples derived from 47,557 subjects, with no previous diagnosis of celiac disease. From this set, 408 undiagnosed cases were detected. To apply ML in a retrospective study, they developed various ML-based predictive models, employing several approaches such as LR, EN, tree-based models with and without boosting and/or bagging, SVM with radial basis functions, ANN, RF, and LDA. The performances of all the developed models were assessed by applying the calculation of the area under the ROC curve. Ten models were trained considering the images set including and excluding variables, and a predictor set including sex, age, number of symptoms, history of any autoimmune condition, thyroid disorder, anemia, hypothyroidism, previous indication to test for celiac disease, dyspepsia, and recurring abdominal pain. Unfortunately, by using this approach, the authors obtained MLbased models with limited discriminatory power, showing an area under the ROC curve ranging from 0.49 to 0.53 . Two models ( $\mathrm{RF}$ and bagged classification trees) showed better performance with respect to the random chance (likelihood $>95 \%$ ), although the predictive power showed a slight improvement compared to the other models. The partial failure in developing effective ML-based models can probably be ascribable to the subtle symptoms in atypical cases, suggesting that considering the mentioned variables for developing predictive models could be impractical, since they did not characterize undiagnosed celiac disease [318]. Accordingly, for improving diagnostic rates, other approaches must be investigated for detecting celiac disease, and recently, Koh and coworkers developed a new ML algorithm for an automated classification of duodenal biopsy images, aiding clinicians to detect celiac disorder and the severity of villous atrophy, taking into account the Marsh score. In the first step, the authors performed a pre-process procedure on biopsy images, subjecting images to a Steerable Pyramidal Transform (SPT) for obtaining sub band coefficients. Considering each sub band diverse entropy (Fuzzy entropy, Kapur entropy, Renyi entropy, Shannon entropy, Vajda entropy, Yager entropy), nonlinear features were calculated and used as input to the decision tree (DT), k-NN, SVM, Adaboost M1 for two classes and Adaboost M2 for multiclass classification, and Bagged Trees and Discriminant Subspace for automatically classifying the extracted features (734 features were extracted from each set of data and so, 26,424 features were extracted from three diverse sets of data) from two classes (normal and celiac) and multiclass (diverse degree of severity of villous atrophy considering Marsh scores) biopsy images. Interestingly, for avoiding the bias created by data imbalance, the authors employed an adaptive synthetic sampling 
(AdaSyn) technique. Next, the authors employed a ten-fold cross-validation approach for training and testing the model. In the ten-fold scheme, the set was divided into ten parts, where nine parts were employed to train the model and one part for testing. Consequently, a different part was utilized to test the model, while the other nine parts were used for training. This procedure was repeated ten times for each part. The performance of the developed ML model was evaluated, and the results showed an accuracy, sensitivity, and specificity of $88.89 \%, 89.67 \%$, and $86.67 \%$ in the two-class classification of two sets of data (Marsh I + II and Marsh III) of Hematoxylin-Eosin-DAB (HED) biopsy images. Furthermore, $82.92 \%$ accuracy, $85.67 \%$ sensitivity, and $76.67 \%$ specificity results were achieved in the two-class classification of two sets of data (Marsh I + II and Marsh III) of RGB biopsy images. Considering the results of multi-class classification (three sets of data), an accuracy of $72 \%$ was obtained for HED biopsy images employing SVM. The suggested approach for an automatic classification of biopsy pictures can help with the process of evaluating villous atrophy using the Marsh score, suggesting that automation of biopsy images is a feasible task. Nevertheless, more data with improved quality (e.g., well-orientated biopsy images) are needed to appropriately train the model, enhancing its predictive power [319]. Remarkably, the reported results have shown great potential for $\mathrm{AI} / \mathrm{ML}$ in the automation of biopsy images for detecting celiac disease, as well as other disorders. Finally, we discuss a recent article in which ML based on the DL technique was adopted for detecting Helicobacter pylori, considering gastric biopsies. Klein and colleagues reported for the first time a computer-based approach for accelerating the recognition of Helicobacter pylori on histological samples. They developed a DL decision support algorithm to be employed on conventional images of gastric biopsies for detecting H. pylori on H\&Eand Giemsa-stained slide images. The latter were classified using a DNN algorithm trained with Giemsa and H\&E slides (191 H\&E-stained and 286 Giemsa-stained slides, for a total of 2629 tiles containing Giemsa and $790 \mathrm{H \& E}$; additionally, 4241 (Giemsa) and 1533 (H\&E) tiles without Helicobacter pylori-like bacterial structures). Several validation approaches presented in the work showed a significant area under the ROC curve $>0.8$, indicating the ability of the model to detect Helicobacter pylori, indicating that AI/ML tools can assist clinicians to formulate a more accurate diagnosis regarding the presence of $H$. pylori on gastric biopsies [320]. 
Table 8. Main examples of AI/ML in gastroenterology.

\begin{tabular}{|c|c|c|c|c|c|}
\hline AI Technique & Target & Dataset & Statistical Parameters & Outcomes & Ref \\
\hline ML algorithm & $\begin{array}{l}\text { Approach for detecting small } \\
(<5 \mathrm{~mm}) \text { adenomatous or } \\
\text { sessile polyps }\end{array}$ & $\begin{array}{l}\text { The ML-based model was trained with } \\
\text { data from } 325 \text { subjects presenting } \\
466 \text { microscopic polyps }\end{array}$ & $\begin{array}{c}\text { The model showed an accuracy of } 94 \% \\
\text { (negative predictive value } 96 \% \text { ), with a } \\
\text { pathologic prediction rate of } 98.1 \% \\
(457 / 466)\end{array}$ & $\begin{array}{l}\text { The application of ML models can be } \\
\text { useful for assisting clinicians in detecting } \\
\text { gastric pathological state }\end{array}$ & [307] \\
\hline $\begin{array}{l}\text { ML model based on } \\
\text { DL algorithm }\end{array}$ & $\begin{array}{l}\text { Approach for detecting polyps in } \\
\text { clinical colonoscopy } \\
\text { investigations }\end{array}$ & $\begin{array}{l}\text { The model was trained using data from } \\
1290 \text { patients ( } 5545 \text { colonoscopy images } \\
\text { containing polyps and images with no } \\
\text { polyps). Validation set of } \\
27,113 \text { colonoscopy images of } \\
1138 \text { patients with one detected polyp }\end{array}$ & $\begin{array}{c}\text { The model showed sensitivity of } 94.38 \% \text {, } \\
\text { specificity of } 95.92 \% \text {, area under the ROC } \\
\text { curve of } 0.984\end{array}$ & $\begin{array}{l}\text { The developed DL model has great } \\
\text { potential in assisting clinicians while } \\
\text { conducting colonoscopy, being able to } \\
\text { correctly discriminate polyps } \\
\text { and adenomas }\end{array}$ & [308] \\
\hline $\begin{array}{l}\text { ML model based on deep } \\
\text { CNN technique }\end{array}$ & $\begin{array}{l}\text { Approach for real-time } \\
\text { evaluation of endoscopic video } \\
\text { images of colorectal polyps }\end{array}$ & $\begin{array}{l}\text { The model was trained and validated } \\
\text { using untouched video data derived from } \\
\text { routine clinical investigations. An } \\
\text { independent set of } 125 \text { videos was used } \\
\text { for the validation } \\
\text { The ML model was trained used }\end{array}$ & $\begin{array}{l}\text { The ML model showed a sensitivity of } \\
98 \% \text {, a specificity of } 83 \% \text {, and an accuracy } \\
\text { of } 94 \%\end{array}$ & $\begin{array}{l}\text { The model was able to discriminate } \\
\text { hyperplastic from adenomatous polyps }\end{array}$ & [309] \\
\hline $\begin{array}{l}\text { ML model based on deep } \\
\text { CNN technique }\end{array}$ & $\begin{array}{l}\text { Approach for detecting polyps } \\
\text { from colonoscopy exams }\end{array}$ & $\begin{array}{l}8641 \text { hand-labeled images, with } \\
4088 \text { unique polyps, from colonoscopy } \\
\text { derived from over } 2000 \text { subjects. The } \\
\text { authors tested the model using } \\
20 \text { colonoscopy videos ( } 5 \mathrm{~h} \text { of duration) }\end{array}$ & $\begin{array}{c}\text { The model showed an area under the } \\
\text { ROC curve of } 0.991 \text { and an accuracy } \\
\text { of } 96.4 \%\end{array}$ & $\begin{array}{l}\text { The CNN algorithm identified a number } \\
\text { of polyps higher than that observed from } \\
\text { expert clinicians }\end{array}$ & [310] \\
\hline $\begin{array}{l}\text { ML tool based on } \\
\text { DNN algorithm }\end{array}$ & $\begin{array}{l}\text { Approach for diagnosing the } \\
\text { invasion depth of ESCC }\end{array}$ & $\begin{array}{l}\text { The model was built using a training set } \\
\text { of } 8660 \text { non-magnified endoscopic images } \\
\text { and } 5678 \text { magnified images from } \\
804 \text { patients with superficial ESCC } \\
\text { presenting cancer invasion. Validation set } \\
\text { consisted of } 405 \text { non-magnified ad } \\
509 \text { magnified images from } 155 \text { subjects }\end{array}$ & $\begin{array}{c}\text { The model showed specificity } 95.8 \% \text {, } \\
\text { sensitivity } 90.1 \% \text {, accuracy } 91 \% \text {, positive } \\
\text { predicted value } 99.2 \% \\
\text { negative predictive value } 63.9 \%\end{array}$ & $\begin{array}{l}\text { These parameters highlighted the } \\
\text { capacity of the model to detect invasion } \\
\text { depth in patients with superficial ESCC }\end{array}$ & [312] \\
\hline ML tool based on CNN algorithm & $\begin{array}{l}\text { Approach for assessing the } \\
\text { severity of IBD and improving } \\
\text { its classification }\end{array}$ & $\begin{array}{l}\text { The model was trained on } \\
26,304 \text { colonoscopy images derived from } \\
841 \text { subjects with ulcerative colitis. The } \\
\text { model was assessed using an } \\
\text { independent test set ( } 3981 \text { images from } \\
114 \text { patients with ulcerative colitis) }\end{array}$ & $\begin{array}{l}\text { The validation was achieved by } \\
\text { calculating the areas under the ROC } \\
\text { curve, and the results for the ML-based } \\
\text { model were } 0.86 \text { and } 0.98 \text { in identifying } \\
\text { normal mucosa (Mayo 0) and mucosal } \\
\text { healing state (Mayo } 0-1 \text { ) }\end{array}$ & $\begin{array}{l}\text { This work indicated that the CNN } \\
\text { algorithm can assist clinicians in } \\
\text { determining severity-based therapies as } \\
\text { well as follow-up endoscopy waits } \\
\text { for IBD }\end{array}$ & [313] \\
\hline
\end{tabular}


Table 8. Cont.

\begin{tabular}{|c|c|c|c|c|c|}
\hline AI Technique & Target & Dataset & $\begin{array}{l}\text { Statistical } \\
\text { Parameters }\end{array}$ & Outcomes & Ref \\
\hline ML model & $\begin{array}{l}\text { ML model for classifying } \\
\text { pediatric IBD }\end{array}$ & $\begin{array}{l}\text { The model was trained using data } \\
\text { derived from endoscopic and histological } \\
\text { imaging of } 287 \text { children affected by IBD }\end{array}$ & $\begin{array}{c}\text { Three supervised ML models showed a } \\
\text { classification accuracy of } 71.0 \%, 76.9 \% \text {, } \\
\text { and } 82.7 \% \text {. The most promising ML } \\
\text { model properly classified patients } \\
\text { (accuracy of } 83.3 \% \text { ) }\end{array}$ & $\begin{array}{l}\text { This work indicated that for the } \\
\text { development of a proper model, it is } \\
\text { necessary to consider both endoscopic } \\
\text { and histological data for a more accurate } \\
\text { disease classification }\end{array}$ & [314] \\
\hline ML model & $\begin{array}{l}\text { Approach for detecting subjects } \\
\text { presenting celiac disease and for } \\
\text { classifying the disorder }\end{array}$ & $\begin{array}{l}\text { The model was trained using } \\
612 \text { endoscopic images from pediatric } \\
\text { patients considering a two-class issue: } \\
\text { mucosa affected by celiac disease and } \\
\text { unaffected duodenal tissue }\end{array}$ & $\begin{array}{c}\text { The model discriminated celiac disease } \\
\text { with an overall accuracy of } 88 \% \text {. The } \\
\text { model showed a reduced accuracy } \\
(63.7 \%) \text { in classifying the severity of } \\
\text { disorders }\end{array}$ & $\begin{array}{l}\text { The classification method was able to } \\
\text { discriminate celiac disease into two } \\
\text { groups (disease vs. no disease) }\end{array}$ & [316] \\
\hline $\mathrm{CNN}$ transfer-learning & $\begin{array}{l}\text { Approach for classifying luminal } \\
\text { endoscopic images of } \\
\text { celiac disease }\end{array}$ & $\begin{array}{c}\text { The training set was composed of } \\
1661 \text { images from luminal endoscopic } \\
\text { data }\end{array}$ & $\begin{array}{l}\text { The model showed an accuracy of } 90.5 \% \\
\text { in identifying celiac disease considering } \\
\text { endoscopic images alone }\end{array}$ & $\begin{array}{l}\text { ML could offer a new method in } \\
\text { diagnostic settings, especially where } \\
\text { acquiring biopsies is complicated }\end{array}$ & [317] \\
\hline ML-based models & $\begin{array}{l}\text { Approach for detecting } \\
\text { undiagnosed celiac disease }\end{array}$ & $\begin{array}{l}\text { The training set was composed of serum } \\
\text { samples derived from } 47,557 \text { subjects, } \\
\text { whit no previous diagnosis of } \\
\text { celiac disease }\end{array}$ & $\begin{array}{l}\text { The models showed an area under the } \\
\text { ROC curve ranging from } 0.49 \text { to } 0.53 \text {. } \\
\text { Two models (RF and bagged classification } \\
\text { trees) showed better performance } \\
\text { (likelihood }>95 \% \text { ) }\end{array}$ & $\begin{array}{l}\text { Considering the selected variables, the } \\
\text { development of predictive models could } \\
\text { be impractical, since they did not } \\
\text { characterize undiagnosed celiac disease }\end{array}$ & [318] \\
\hline ML algorithm & $\begin{array}{l}\text { Approach for an automated } \\
\text { classification of duodenal } \\
\text { biopsy images }\end{array}$ & $\begin{array}{l}\text { The model was trained using biopsy } \\
\text { images extracting features ( } 734 \text { features } \\
\text { from each set of data and so, } \\
\text { 26,424 features were extracted from three } \\
\text { diverse sets of data) from two classes } \\
\text { (normal and celiac) }\end{array}$ & $\begin{array}{l}\text { The model showed: accuracy of } 88.89 \% \\
\text { sensitivity of } 89.67 \% \text { specificity of } 86.67 \% \\
\text { in the two-class classification }\end{array}$ & $\begin{array}{l}\text { The approach for an automatic } \\
\text { classification of biopsy pictures can help } \\
\text { with the process of evaluating villous } \\
\text { atrophy, suggesting that automation of } \\
\text { biopsy images is a feasible task }\end{array}$ & [319] \\
\hline $\begin{array}{l}\text { DL decision support method } \\
\text { based on DNN algorithm }\end{array}$ & $\begin{array}{l}\text { Approach for detecting } \\
\text { Helicobacter pylori considering } \\
\text { gastric biopsies }\end{array}$ & $\begin{array}{l}\text { The model was trained considering } \\
\text { Giemsa and H\&E slides (191 H\&E- and } \\
286 \text { Giemsa-stained slides for a total of } \\
2629 \text { tiles containing Giemsa and } \\
790 \text { H\&E; additionally, } 4241 \text { (Giemsa) and } \\
1533 \text { (H\&E) tiles without } H \text {. pylori-like } \\
\text { bacterial structures) }\end{array}$ & $\begin{array}{c}\text { Several validation approaches were used } \\
\text { showing an area under the ROC } \\
\text { curve }>0.8\end{array}$ & $\begin{array}{l}\text { The model was able to detect } H . \text { pylori, } \\
\text { indicating that ML tools can assist } \\
\text { clinicians in diagnosis regarding the } \\
\text { presence of } H . \text { pylori in gastric biopsies }\end{array}$ & [320] \\
\hline
\end{tabular}

RF- random forest; ROC—-receiver operating characteristic. 


\subsubsection{AI in Dermatology}

As discussed for different medical fields, the translational power of AI/ML in medicine is great. From diagnosis to targeted therapy, ML techniques have great potential to improve dermatologists' practices. Current progress in computing, along with the availability of huge datasets (e.g., image and -omics databases, electronic medical records), has spurred the development of ML-based approaches in dermatology [136,321]. Some relevant examples were analyzed here (Table 9). Spyridonos and coworkers described a computational approach for discriminating actinic keratoses from healthy skin based on color texture examination of typical clinical photographs. It is important to recognize such skin lesions early, since they are frequent pre-malignant injuries that indicate the possibility of developing invasive skin squamous cell carcinoma. They collected non-standardized clinical photographs of 22 patients of both actinic keratoses and healthy skin, labeled by experienced dermatologists, highlighting ROI. In this way, the authors obtained a dataset composed of 6010 (actinic keratoses) and 13,915 (healthy) ROI. Information about color texture was obtained by employing local binary patterns (LBP) or texton frequency histograms and assessed using a classifier based on the SVM technique. The classification method was evaluated by employing the leave-one-patient-out procedure in RGB, YIQ, and CIELab color spaces. The best performing configuration of the SVM model was tested using 157 actinic keratoses and 216 healthy skin rectangular regions of arbitrary size. The actinic keratoses treatment outcome was assessed in a further group of eight subjects with 32 skin lesions. The excellent configuration for discriminating the samples was obtained using LBP color texture descriptors estimated from Y and I of the YIQ color space, and the SVM model achieved a sensitivity of $80.1 \%$ and a specificity of $81.1 \%$ at ROI level, while a sensitivity of $89.8 \%$ and a specificity of $91.7 \%$ at region level. The authors observed a quantitative actinic keratoses reduction of $83.6 \%$ considering the classifier used. Interestingly, this work indicated that a combination of clinical photographs with the ML algorithm for a detailed image analysis represents a useful, non-invasive, cost-effective approach to monitor actinic keratoses for early therapeutic strategies against such skin lesions [322]. Intriguingly, some AI-based models have been established for predicting skin sensitization. In this context, Tsujita-Inoue and collaborators developed an ML approach based on ANN algorithm for assessing the skin sensitization risk derived from several chemicals. The authors used several descriptors (e.g., data from antioxidant response element (ARE) tests and LogP, indicating lipid solubility and skin absorption) for implementing a previous version of a software able to predict the murine local lymph node assay (LLNA) test results [323]. In fact, LLNA is the most used in vivo method to assess the sensitizing potential of chemical entities. Accordingly, they developed iSENS ver.2. The authors used the data obtained for 62 compounds in murine LLNA tests. Among them, 53 composed the training set, while the others were employed for validating the developed computational tool. The predictivity of the ANN-based model was assessed by employing a 10-fold cross-validation method. The accuracy, specificity, and sensitivity of the computational model were $84.9 \%, 92.3 \%$ and $82.5 \%$, respectively [324]. According to the results, ML approaches for evaluating the risk estimation of compounds regarding skin sensitization can represent a valuable resource for replacing animal testing. Subsequently, Zang and collaborators improved the number of selected chemicals for developing an ML model to predict the skin sensitization, considering two datasets, one including LLNA results regarding 120 chemicals and the other covering human skin sensitization results taking into account 87 chemicals (all these substances were included in the LLNA dataset). Moreover, the authors included six physicochemical features of these chemicals related to skin exposure and penetration (octanol/water partition coefficient, water solubility, vapor pressure, melting point, boiling point, and molecular weight). The molecules were distributed into the training set (75\%) and test set $(25 \%)$. Different ML approaches were used for developing predictive models, including classification and regression tree, LDA, LR, and SVM. The validation step was performed by applying the leave-one-out cross-validation procedure. SVM was found to be the best method in modeling LLNA output with an accuracy of $89 \%$, sensitivity of 
$86 \%$, and specificity of $92 \%$ on the test set. Regarding the prediction for human outcomes, SVM model demonstrated an accuracy of $81 \%$, a sensitivity and specificity of $86 \%$, and $78 \%$, respectively [325]. Another area of dermatology regards skin lesions and malignancies. Esteva and coworkers generated a deep CNN-based model for classifying skin lesions. They trained a CNN model, employing a set of 129,450 clinical images enclosing 2032 diverse disorders, matching the performance of 21 dermatologists experienced across three serious diagnoses: keratinocyte carcinoma classification, melanoma classification and melanoma classification by means of dermoscopic data. The results show an area under the ROC curve of 0.96 for carcinoma, and of 0.94 for melanoma [326]. Haenssle and colleagues, in an interesting experiment, evaluated the accuracy of melanoma skin cancer diagnosis considering the performance of 58 experts in comparison with the assessment performed by an ML-based model generated using the CNN technique. The ML model was developed, validated, and tested for classifying dermoscopic images of lesions of melanocytic origin (melanoma, benign nevi) for diagnostic purposes. The dataset enclosed a test set composed of 300 images containing $20 \%$ melanomas (in situ and invasive) of all body sites and of all common histotypes, and $80 \%$ benign melanocytic nevi. The average of the calculated area under the ROC curves was 0.79 , considering the results from the 58 dermatologists, and 0.86 , considering the ML model, respectively, indicating an improvement concerning the diagnostic performance derived from the application of the computer-based tool. Accordingly, the study highlighted that appropriately trained ML models have the ability to perform accurate diagnostic classification of dermoscopic images of melanocytic origin $[327,328]$. Han and coworkers developed an ML model using the CNN algorithm for classifying clinical images from 12 skin diseases (basal cell carcinoma, squamous cell carcinoma, intraepithelial carcinoma, melanocytic nevus, pyogenic granuloma, seborrheic keratosis, actinic keratosis, wart, malignant melanoma, hemangioma, lentigo, and dermatofibroma). The ML model was trained, tested, and validated employing the Asan dataset, MED-NODE dataset, and atlas site images, for a total of 19,398 images, divided into the training set and test set. Considering the Asan dataset, the area under the ROC curve concerning the diagnosis of basal cell carcinoma, squamous cell carcinoma, intraepithelial carcinoma, and melanoma was $0.96,0.83,0.82$, and 0.96 , respectively. Considering the Edinburgh dataset, the area under the ROC curve for the same disorders was 0.90, 0.91, 0.83 , and 0.88 , respectively. The developed ML-based model demonstrated comparable performances to those obtained from 16 dermatologists. Furthermore, as indicated by the authors, for improving the performance of the CNN algorithm, supplementary images representing a larger variety of ages and ethnicities should be employed [329]. Following this trend, other studies have employed data from dermoscopic images sometimes combined with macroscopic images for training supervised or unsupervised ML models, based principally on $\mathrm{CNN}$ algorithms to detect and/or classify cutaneous malignancies, including melanoma and basal cell carcinoma [330-337]. Notably, CNN algorithms showed interesting performances also in classifying and detecting other relevant dermatological disorders, including onychomycosis, rosacea, atopic dermatitis, and psoriasis [338-344]. 
Table 9. Main examples of AI/ML in dermatology.

\begin{tabular}{|c|c|c|c|c|c|}
\hline AI Technique & Target & Dataset & Statistical Parameters & Outcomes & Ref \\
\hline $\begin{array}{l}\text { ML model based on } \\
\text { SVM technique }\end{array}$ & $\begin{array}{l}\text { Computational approach for } \\
\text { discriminating actinic keratoses } \\
\text { from healthy skin based on color } \\
\text { texture examination of typical } \\
\text { clinical photographs }\end{array}$ & $\begin{array}{l}\text { Dataset composed of } 6010 \text { (actinic } \\
\text { keratoses) and } 13,915 \text { (healthy) ROI from } \\
22 \text { patients. The model was tested using } \\
157 \text { actinic keratoses and } 216 \text { healthy skin } \\
\text { rectangular regions of arbitrary size }\end{array}$ & $\begin{array}{l}\text { The SVM model achieved a sensitivity of } \\
80.1 \% \text { and a specificity of } 81.1 \% \text { at ROI } \\
\text { level, while a sensitivity of } 89.8 \% \text { and a } \\
\text { specificity of } 91.7 \% \text { at region level }\end{array}$ & $\begin{array}{l}\text { This work indicated that combining } \\
\text { clinical photos with ML algorithms for a } \\
\text { detailed image analysis is a useful, } \\
\text { non-invasive, cost-effective method to } \\
\text { monitor actinic keratoses for early } \\
\text { therapeutic strategies against such } \\
\text { skin lesions }\end{array}$ & [322] \\
\hline $\begin{array}{l}\text { ML approach based on } \\
\text { ANN algorithm }\end{array}$ & $\begin{array}{l}\text { Approach for assessing the skin } \\
\text { sensitization risk derived from } \\
\text { several chemicals }\end{array}$ & $\begin{array}{l}\text { Dataset obtained for } 62 \text { compounds in } \\
\text { murine LLNA ( } 53 \text { composed the training } \\
\text { set, while the others were used for } \\
\text { validating the computational tool) }\end{array}$ & $\begin{array}{c}\text { The model was assessed using a } 10-\text { fold } \\
\text { cross-validation method. The accuracy, } \\
\text { specificity, and sensitivity of the model } \\
\text { were } 84.9 \%, 92.3 \% \text {, and } 82.5 \% \text {, } \\
\text { respectively }\end{array}$ & $\begin{array}{l}\text { ML approaches for evaluating the risk } \\
\text { estimation of compounds regarding skin } \\
\text { sensitization can represent a valuable } \\
\text { resource for replacing animal testing }\end{array}$ & [324] \\
\hline $\begin{array}{l}\text { ML model based on } \\
\text { SVM technique }\end{array}$ & $\begin{array}{l}\text { Approach for assessing the skin } \\
\text { sensitization risk derived from } \\
\text { several chemicals }\end{array}$ & $\begin{array}{l}\text { Dataset composed of } 120 \text { chemicals with } \\
\text { data on human skin sensitization, } \\
\text { including LLNA. The molecules were } \\
\text { distributed into the training set }(75 \%) \text { and } \\
\text { test set }(25 \%)\end{array}$ & $\begin{array}{l}\text { The validation step was performed } \\
\text { applying LOO-cv. SVM was found to be } \\
\text { the best method in modeling LLNA } \\
\text { output with an accuracy of } 89 \% \text { and a } \\
\text { sensitivity of } 86 \% \text {, and specificity of } 92 \% \\
\text { on the test set }\end{array}$ & $\begin{array}{l}\text { SVM model showed interesting results } \\
\text { regarding the prediction of } \\
\text { human outcomes }\end{array}$ & [325] \\
\hline Deep CNN-based model & $\begin{array}{l}\text { Approach for classifying } \\
\text { skin lesions }\end{array}$ & $\begin{array}{l}\text { The model was trained using a set of } \\
129,450 \text { clinical images }\end{array}$ & $\begin{array}{l}\text { Results show an area under the ROC } \\
\text { curve of } 0.96 \text { for carcinoma, and of } 0.94 \\
\text { for melanoma }\end{array}$ & $\begin{array}{l}\text { Computational tools based on CNN } \\
\text { algorithms correctly classified skin lesions }\end{array}$ & [326] \\
\hline $\begin{array}{l}\text { ML model using } \\
\text { CNN algorithm }\end{array}$ & $\begin{array}{l}\text { Approach for classifying clinical } \\
\text { images from } 12 \text { skin diseases }\end{array}$ & $\begin{array}{l}\text { ML model was trained, tested, and } \\
\text { validated employing the Asan dataset, } \\
\text { MED-NODE dataset, and atlas site } \\
\text { images, for a total of 19,398 images, } \\
\text { opportunely divided in training set and } \\
\text { test set }\end{array}$ & $\begin{array}{l}\text { Considering the Asan dataset, the area } \\
\text { under the ROC curve concerning the } \\
\text { diagnosis of basal cell carcinoma, } \\
\text { squamous cell carcinoma, intraepithelial } \\
\text { carcinoma, and melanoma was } 0.96,0.83 \text {, } \\
0.82 \text {, and } 0.96 \text {, respectively. Considering } \\
\text { the Edinburgh dataset, the area under the } \\
\text { ROC curve for the same disorders was } \\
0.90,0.91,0.83 \text {, and } 0.88 \text {, respectively. }\end{array}$ & $\begin{array}{l}\text { The ML-based model showed } \\
\text { comparable performances to those } \\
\text { obtained from } 16 \text { dermatologists }\end{array}$ & [329] \\
\hline
\end{tabular}

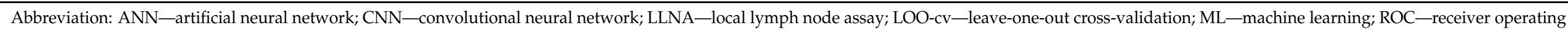
characteristic; ROI—regions of interest; SVM—support vector machine. 


\section{Conclusions and Future Perspective}

$\mathrm{AI} / \mathrm{ML}$ has reemerged in recent years as a powerful set of tools for unlocking value from big datasets. The extraordinary increase in the use of AI and ML techniques in almost all fields of technology, science, and medicine clearly indicates a significantly greater role for these procedures in the discovery of innovative therapies in the near future. The above descriptive examples demonstrate how useful these methodologies can be in discovering novel drug candidates, biomarkers, and drug targets, as well as for detecting and evaluating the progression of a given disease. It is also clear from the literature that the rate of adoption of these methods is increasing significantly. This is determined by the increase in the usage of high-throughput screens, increased power and availability of open-source ML methods, and the development of new AI/ML algorithms, generating more accurate descriptors and model relationships. Remarkably, the quality of the generated ML algorithms is also principally defined by the quality of the input data, so proper data acquisition and curation are crucial steps for developing predictive/effective ML-based models. In the context of ML as a new diagnostic technique and for identifying appropriate therapeutic regimens, most of the developed models were found to outperform current clinical standards based on the assessment of sensitivity, specificity, and accuracy, employing the ROC method for comparing ML algorithms and clinician performances. This validation undoubtedly added validity to model performances, but for a real-world assessment, any new methodology employed in clinical settings should demonstrate superior performance in properly designed, randomized clinical trials. Nonetheless, advances in ML will provide, in the near future, effective methods for addressing the uncertainty observed in translational medicine, facilitating more forceful, data-driven decision making for developing the next generation of diagnostic tools and therapeutic agents.

Author Contributions: Data curation, V.C. and S.B.; writing—original draft preparation, S.B.; writing-review and editing, V.C. and S.B.; supervision, S.B. All authors have read and agreed to the published version of the manuscript.

Funding: This research received no external funding.

Institutional Review Board Statement: Not applicable.

Informed Consent Statement: Not applicable.

Data Availability Statement: Not applicable.

Conflicts of Interest: The authors declare no conflict of interest.

\section{References}

1. Available online: https://www.brookings.edu/techstream/what-investment-trends-reveal-about-the-global-ai-landscape/ (accessed on 14 October 2021).

2. Available online: https:// outsideinsight.com/insights/global-ai-investment-150-billion-2025/ (accessed on 14 October 2021).

3. Hinton, G. Deep Learning-A Technology With the Potential to Transform Health Care. JAMA 2018, 320, 1101-1102. [CrossRef]

4. Esteva, A.; Robicquet, A.; Ramsundar, B.; Kuleshov, V.; DePristo, M.; Chou, K.; Cui, C.; Corrado, G.; Thrun, S.; Dean, J. A guide to deep learning in healthcare. Nat. Med. 2019, 25, 24-29. [CrossRef]

5. He, J.; Baxter, S.L.; Xu, J.; Xu, J.; Zhou, X.; Zhang, K. The practical implementation of artificial intelligence technologies in medicine. Nat. Med. 2019, 25, 30-36. [CrossRef]

6. Toh, T.S.; Dondelinger, F.; Wang, D. Looking beyond the hype: Applied AI and machine learning in translational medicine. EBioMedicine 2019, 47, 607-615. [CrossRef]

7. Available online: https://www.fda.gov/medical-devices/software-medical-device-samd/artificial-intelligence-and-machinelearning-software-medical-device\#whatis (accessed on 14 October 2021).

8. Murdoch, T.B.; Detsky, A.S. The inevitable application of big data to health care. JAMA 2013, 309, 1351-1352. [CrossRef]

9. Jiang, F.; Jiang, Y.; Zhi, H.; Dong, Y.; Li, H.; Ma, S.; Wang, Y.; Dong, Q.; Shen, H.; Wang, Y. Artificial intelligence in healthcare: Past, present and future. Stroke Vasc. Neurol. 2017, 2, 230-243. [CrossRef] [PubMed]

10. Rajkomar, A.; Dean, J.; Kohane, I. Machine Learning in Medicine. N. Engl. J. Med. 2019, 380, 1347-1358. [CrossRef] [PubMed]

11. Beam, A.L.; Kohane, I.S. Big Data and Machine Learning in Health Care. JAMA 2018, 319, 1317-1318. [CrossRef]

12. Muhammad, L.J.; Algehyne, E.A.; Usman, S.S.; Ahmad, A.; Chakraborty, C.; Mohammed, I.A. Supervised Machine Learning Models for Prediction of COVID-19 Infection using Epidemiology Dataset. SN Comput. Sci. 2021, 2, 11. [CrossRef] 
13. Mirri, S.; Delnevo, G.; Roccetti, M. Is a COVID-19 Second Wave Possible in Emilia-Romagna (Italy)? Forecasting a Future Outbreak with Particulate Pollution and Machine Learning. Computation 2020, 8, 74. [CrossRef]

14. Patel, L.; Shukla, T.; Huang, X.; Ussery, D.W.; Wang, S. Machine Learning Methods in Drug Discovery. Molecules 2020, 25, 5277. [CrossRef] [PubMed]

15. Reda, C.; Kaufmann, E.; Delahaye-Duriez, A. Machine learning applications in drug development. Comput. Struct. Biotechnol. J. 2020, 18, 241-252. [CrossRef]

16. Gupta, R.; Srivastava, D.; Sahu, M.; Tiwari, S.; Ambasta, R.K.; Kumar, P. Artificial intelligence to deep learning: Machine intelligence approach for drug discovery. Mol. Divers. 2021, 25, 1315-1360. [CrossRef]

17. Ramesh, N.; Tasdizen, T. Detection and segmentation in microscopy images. In Computer Vision for Microscopy Image Analysis; Academic Press Books; Elsevier: New York, NY, USA, 2021; pp. 43-71. [CrossRef]

18. Alloghani, M.; Al-Jumeily, D.; Mustafina, J.; Hussain, A.; Aljaaf, A.J. A Systematic Review on Supervised and Unsupervised Machine Learning Algorithms for Data Science. In Supervised and Unsupervised Learning for Data Science; Springer Nature: London, UK, 2020; pp. 3-21. [CrossRef]

19. Cleret de Langavant, L.; Bayen, E.; Yaffe, K. Unsupervised Machine Learning to Identify High Likelihood of Dementia in Population-Based Surveys: Development and Validation Study. J. Med. Internet Res. 2018, 20, e10493. [CrossRef] [PubMed]

20. Lu, C.; Ghoman, S.K.; Cutumisu, M.; Schmolzer, G.M. Unsupervised Machine Learning Algorithms Examine Healthcare Providers' Perceptions and Longitudinal Performance in a Digital Neonatal Resuscitation Simulator. Front. Pediatr. 2020, 8, 544. [CrossRef]

21. Roohi, A.; Faust, K.; Djuric, U.; Diamandis, P. Unsupervised Machine Learning in Pathology: The Next Frontier. Surg. Pathol. Clin. 2020, 13, 349-358. [CrossRef]

22. Lopez, C.; Tucker, S.; Salameh, T.; Tucker, C. An unsupervised machine learning method for discovering patient clusters based on genetic signatures. J. Biomed. Inf. 2018, 85, 30-39. [CrossRef]

23. Ceriotti, M. Unsupervised machine learning in atomistic simulations, between predictions and understanding. J. Chem. Phys. 2019, 150, 150901. [CrossRef]

24. Ma, E.Y.; Kim, J.W.; Lee, Y.; Cho, S.W.; Kim, H.; Kim, J.K. Combined unsupervised-supervised machine learning for phenotyping complex diseases with its application to obstructive sleep apnea. Sci. Rep. 2021, 11, 4457. [CrossRef]

25. Omta, W.A.; van Heesbeen, R.G.; Shen, I.; de Nobel, J.; Robers, D.; van der Velden, L.M.; Medema, R.H.; Siebes, A.; Feelders, A.J.; Brinkkemper, S.; et al. Combining Supervised and Unsupervised Machine Learning Methods for Phenotypic Functional Genomics Screening. SLAS Discov. 2020, 25, 655-664. [CrossRef] [PubMed]

26. Neftci, E.O.; Averbeck, B.B. Reinforcement learning in artificial and biological systems. Nat. Mach. Intell. 2019, 1, 133-143. [CrossRef]

27. Yauney, G.; Shah, P. Reinforcement Learning with Action-Derived Rewards for Chemotherapy and Clinical Trial Dosing Regimen Selection. In Proceedings of the 3rd Machine Learning for Healthcare Conference, Palo Alto, CA, USA, 17-18 August 2018; pp. 161-226.

28. Sirous, H.; Campiani, G.; Brogi, S.; Calderone, V.; Chemi, G. Computer-Driven Development of an in Silico Tool for Finding Selective Histone Deacetylase 1 Inhibitors. Molecules 2020, 25, 1952. [CrossRef]

29. Brogi, S.; Brindisi, M.; Joshi, B.P.; Sanna Coccone, S.; Parapini, S.; Basilico, N.; Novellino, E.; Campiani, G.; Gemma, S.; Butini, S. Exploring clotrimazole-based pharmacophore: 3D-QSAR studies and synthesis of novel antiplasmodial agents. Bioorg Med. Chem. Lett. 2015, 25, 5412-5418. [CrossRef] [PubMed]

30. Brogi, S.; Papazafiri, P.; Roussis, V.; Tafi, A. 3D-QSAR using pharmacophore-based alignment and virtual screening for discovery of novel MCF-7 cell line inhibitors. Eur. J. Med. Chem. 2013, 67, 344-351. [CrossRef] [PubMed]

31. Brogi, S.; Kladi, M.; Vagias, C.; Papazafiri, P.; Roussis, V.; Tafi, A. Pharmacophore modeling for qualitative prediction of antiestrogenic activity. J. Chem. Inf. Model. 2009, 49, 2489-2497. [CrossRef]

32. Muratov, E.N.; Bajorath, J.; Sheridan, R.P.; Tetko, I.V.; Filimonov, D.; Poroikov, V.; Oprea, T.I.; Baskin, I.I.; Varnek, A.; Roitberg, A.; et al. QSAR without borders. Chem. Soc. Rev. 2020, 49, 3525-3564. [CrossRef]

33. Lin, X.; Li, X.; Lin, X. A Review on Applications of Computational Methods in Drug Screening and Design. Molecules 2020, 25, 1375. [CrossRef]

34. Kohlbacher, S.M.; Langer, T.; Seidel, T. QPHAR: Quantitative pharmacophore activity relationship: Method and validation. J. Cheminform. 2021, 13, 57. [CrossRef] [PubMed]

35. Flori, L.; Petrarolo, G.; Brogi, S.; La Motta, C.; Testai, L.; Calderone, V. Identification of novel SIRT1 activators endowed with cardioprotective profile. Eur. J. Pharm. Sci. 2021, 165, 105930. [CrossRef]

36. Sirous, H.; Chemi, G.; Campiani, G.; Brogi, S. An integrated in silico screening strategy for identifying promising disruptors of p53-MDM2 interaction. Comput. Biol. Chem. 2019, 83, 107105. [CrossRef]

37. Battah, B.; Chemi, G.; Butini, S.; Campiani, G.; Brogi, S.; Delogu, G.; Gemma, S. A Repurposing Approach for Uncovering the Anti-Tubercular Activity of FDA-Approved Drugs with Potential Multi-Targeting Profiles. Molecules 2019, 24, 4373. [CrossRef]

38. Sirous, H.; Chemi, G.; Gemma, S.; Butini, S.; Debyser, Z.; Christ, F.; Saghaie, L.; Brogi, S.; Fassihi, A.; Campiani, G.; et al. Identification of Novel 3-Hydroxy-pyran-4-One Derivatives as Potent HIV-1 Integrase Inhibitors Using in silico Structure-Based Combinatorial Library Design Approach. Front. Chem. 2019, 7, 574. [CrossRef]

39. Batool, M.; Ahmad, B.; Choi, S. A Structure-Based Drug Discovery Paradigm. Int. J. Mol. Sci. 2019, 20, 2783. [CrossRef] [PubMed]

40. Maia, E.H.B.; Assis, L.C.; de Oliveira, T.A.; da Silva, A.M.; Taranto, A.G. Structure-Based Virtual Screening: From Classical to Artificial Intelligence. Front. Chem. 2020, 8, 343. [CrossRef] 
41. Fischer, A.; Smiesko, M.; Sellner, M.; Lill, M.A. Decision Making in Structure-Based Drug Discovery: Visual Inspection of Docking Results. J. Med. Chem. 2021, 64, 2489-2500. [CrossRef] [PubMed]

42. De Vivo, M.; Masetti, M.; Bottegoni, G.; Cavalli, A. Role of Molecular Dynamics and Related Methods in Drug Discovery. J. Med. Chem. 2016, 59, 4035-4061. [CrossRef] [PubMed]

43. Decherchi, S.; Grisoni, F.; Tiwary, P.; Cavalli, A. Editorial: Molecular Dynamics and Machine Learning in Drug Discovery. Front. Mol. Biosci. 2021, 8, 673773. [CrossRef]

44. Brogi, S.; Ramalho, T.C.; Kuca, K.; Medina-Franco, J.L.; Valko, M. Editorial: In silico Methods for Drug Design and Discovery. Front. Chem. 2020, 8, 612. [CrossRef]

45. Zaccagnini, L.; Brogi, S.; Brindisi, M.; Gemma, S.; Chemi, G.; Legname, G.; Campiani, G.; Butini, S. Identification of novel fluorescent probes preventing $\operatorname{PrP}(\mathrm{Sc})$ replication in prion diseases. Eur. J. Med. Chem. 2017, 127, 859-873. [CrossRef]

46. Brogi, S. Computational Approaches for Drug Discovery. Molecules 2019, 24, 3061. [CrossRef]

47. Sirous, H.; Campiani, G.; Calderone, V.; Brogi, S. Discovery of novel hit compounds as potential HDAC1 inhibitors: The case of ligand and structure-based virtual screening. Comput. Biol. Med. 2021, 137, 104808. [CrossRef]

48. Vazquez, J.; Lopez, M.; Gibert, E.; Herrero, E.; Luque, F.J. Merging Ligand-Based and Structure-Based Methods in Drug Discovery: An Overview of Combined Virtual Screening Approaches. Molecules 2020, 25, 4723. [CrossRef]

49. Ferreira, L.L.G.; Andricopulo, A.D. Editorial: Chemoinformatics Approaches to Structure- and Ligand-Based Drug Design. Front. Pharm. 2018, 9, 1416. [CrossRef]

50. Ivanov, J.; Polshakov, D.; Kato-Weinstein, J.; Zhou, Q.; Li, Y.; Granet, R.; Garner, L.; Deng, Y.; Liu, C.; Albaiu, D.; et al. Quantitative Structure-Activity Relationship Machine Learning Models and their Applications for Identifying Viral 3CLproand RdRp-Targeting Compounds as Potential Therapeutics for COVID-19 and Related Viral Infections. ACS Omega 2020, 5, 27344-27358. [CrossRef]

51. Ancuceanu, R.; Dinu, M.; Neaga, I.; Laszlo, F.G.; Boda, D. Development of QSAR machine learning-based models to forecast the effect of substances on malignant melanoma cells. Oncol. Lett. 2019, 17, 4188-4196. [CrossRef]

52. Keyvanpour, M.R.; Shirzad, M.B. An Analysis of QSAR Research Based on Machine Learning Concepts. Curr. Drug Discov. Technol. 2021, 18, 17-30. [CrossRef]

53. Simoes, R.S.; Maltarollo, V.G.; Oliveira, P.R.; Honorio, K.M. Transfer and Multi-task Learning in QSAR Modeling: Advances and Challenges. Front. Pharm. 2018, 9, 74. [CrossRef]

54. Lill, M.A. Multi-dimensional QSAR in drug discovery. Drug Discov. Today 2007, 12, 1013-1017. [CrossRef] [PubMed]

55. Sadik, K.; Byadi, S.; Hachim, M.E.; Hamdani, N.E.; Podlipnik, Č.; Aboulmouhajir, A. Multi-QSAR approaches for investigating the relationship between chemical structure descriptors of Thiadiazole derivatives and their corrosion inhibition performance. J. Mol. Struct. 2021, 1240, 130571. [CrossRef]

56. Speck-Planche, A.; Cordeiro, M.N. Multi-Target QSAR Approaches for Modeling Protein Inhibitors. Simultaneous Prediction of Activities Against Biomacromolecules Present in Gram-Negative Bacteria. Curr. Top. Med. Chem. 2015, 15, 1801-1813. [CrossRef] [PubMed]

57. Solimeo, R.; Zhang, J.; Kim, M.; Sedykh, A.; Zhu, H. Predicting chemical ocular toxicity using a combinatorial QSAR approach. Chem. Res. Toxicol. 2012, 25, 2763-2769. [CrossRef]

58. Zhu, H.; Tropsha, A.; Fourches, D.; Varnek, A.; Papa, E.; Gramatica, P.; Oberg, T.; Dao, P.; Cherkasov, A.; Tetko, I.V. Combinatorial QSAR modeling of chemical toxicants tested against Tetrahymena pyriformis. J. Chem. Inf. Model. 2008, 48, 766-784. [CrossRef] [PubMed]

59. Neves, B.J.; Braga, R.C.; Melo-Filho, C.C.; Moreira-Filho, J.T.; Muratov, E.N.; Andrade, C.H. QSAR-Based Virtual Screening: Advances and Applications in Drug Discovery. Front. Pharm. 2018, 9, 1275. [CrossRef] [PubMed]

60. Melo-Filho, C.C.; Dantas, R.F.; Braga, R.C.; Neves, B.J.; Senger, M.R.; Valente, W.C.; Rezende-Neto, J.M.; Chaves, W.T.; Muratov, E.N.; Paveley, R.A.; et al. QSAR-Driven Discovery of Novel Chemical Scaffolds Active against Schistosoma mansoni. J. Chem. Inf. Model. 2016, 56, 1357-1372. [CrossRef]

61. Vamathevan, J.; Clark, D.; Czodrowski, P.; Dunham, I.; Ferran, E.; Lee, G.; Li, B.; Madabhushi, A.; Shah, P.; Spitzer, M.; et al. Applications of machine learning in drug discovery and development. Nat. Rev. Drug Discov. 2019, 18, 463-477. [CrossRef]

62. Dara, S.; Dhamercherla, S.; Jadav, S.S.; Babu, C.M.; Ahsan, M.J. Machine Learning in Drug Discovery: A Review. Artif. Intell. Rev. 2021, 2021, 1-53. [CrossRef]

63. Vignaux, P.A.; Minerali, E.; Foil, D.H.; Puhl, A.C.; Ekins, S. Machine Learning for Discovery of GSK3beta Inhibitors. ACS Omega 2020, 5, 26551-26561. [CrossRef]

64. Huang, D.Z.; Kouznetsova, V.L.; Tsigelny, I.F. Deep-learning- and pharmacophore-based prediction of RAGE inhibitors. Phys. Biol. 2020, 17, 036003. [CrossRef]

65. Shi, C.; Dong, F.; Zhao, G.; Zhu, N.; Lao, X.; Zheng, H. Applications of machine-learning methods for the discovery of NDM-1 inhibitors. Chem. Biol. Drug Des. 2020, 96, 1232-1243. [CrossRef] [PubMed]

66. Tinivella, A.; Pinzi, L.; Rastelli, G. Prediction of activity and selectivity profiles of human Carbonic Anhydrase inhibitors using machine learning classification models. J. Cheminform. 2021, 13, 18. [CrossRef]

67. Ballester, P.J. Machine Learning for Molecular Modelling in Drug Design. Biomolecules 2019, 9, 216. [CrossRef] [PubMed]

68. Medina-Franco, J.L. Grand Challenges of Computer-Aided Drug Design: The Road Ahead. Front. Drug Discov. 2021, 1, 2. 
69. Rodriguez, S.; Hug, C.; Todorov, P.; Moret, N.; Boswell, S.A.; Evans, K.; Zhou, G.; Johnson, N.T.; Hyman, B.T.; Sorger, P.K.; et al. Machine learning identifies candidates for drug repurposing in Alzheimer's disease. Nat. Commun. 2021, 12, 1033. [CrossRef] [PubMed]

70. Tanoli, Z.; Vaha-Koskela, M.; Aittokallio, T. Artificial intelligence, machine learning, and drug repurposing in cancer. Expert Opin. Drug Discov. 2021, 16, 977-989. [CrossRef] [PubMed]

71. Zhou, Y.; Wang, F.; Tang, J.; Nussinov, R.; Cheng, F. Artificial intelligence in COVID-19 drug repurposing. Lancet Digit. Health 2020, 2, e667-e676. [CrossRef]

72. Beck, B.R.; Shin, B.; Choi, Y.; Park, S.; Kang, K. Predicting commercially available antiviral drugs that may act on the novel coronavirus (SARS-CoV-2) through a drug-target interaction deep learning model. Comput. Struct. Biotechnol. J. 2020, 18, 784-790. [CrossRef]

73. Xu, Y.; Verma, D.; Sheridan, R.P.; Liaw, A.; Ma, J.; Marshall, N.M.; McIntosh, J.; Sherer, E.C.; Svetnik, V.; Johnston, J.M. Deep Dive into Machine Learning Models for Protein Engineering. J. Chem. Inf. Model. 2020, 60, 2773-2790. [CrossRef]

74. Gao, W.; Mahajan, S.P.; Sulam, J.; Gray, J.J. Deep Learning in Protein Structural Modeling and Design. Patterns 2020, 1, 100142. [CrossRef]

75. Hameduh, T.; Haddad, Y.; Adam, V.; Heger, Z. Homology modeling in the time of collective and artificial intelligence. Comput. Struct. Biotechnol. J. 2020, 18, 3494-3506. [CrossRef]

76. Torrisi, M.; Pollastri, G.; Le, Q. Deep learning methods in protein structure prediction. Comput. Struct. Biotechnol. J. 2020, 18, 1301-1310. [CrossRef]

77. Lavecchia, A. Machine-learning approaches in drug discovery: Methods and applications. Drug Discov. Today 2015, 20, 318-331. [CrossRef] [PubMed]

78. Gawehn, E.; Hiss, J.A.; Schneider, G. Deep Learning in Drug Discovery. Mol. Inf. 2016, 35, 3-14. [CrossRef]

79. Kimber, T.B.; Chen, Y.; Volkamer, A. Deep Learning in Virtual Screening: Recent Applications and Developments. Int. J. Mol. Sci. 2021, 22, 4435. [CrossRef] [PubMed]

80. Melville, J.L.; Burke, E.K.; Hirst, J.D. Machine learning in virtual screening. Comb. Chem. High Throughput Screen. 2009, 12, 332-343. [CrossRef]

81. Mendez, D.; Gaulton, A.; Bento, A.P.; Chambers, J.; De Veij, M.; Felix, E.; Magarinos, M.P.; Mosquera, J.F.; Mutowo, P.; Nowotka, M.; et al. ChEMBL: Towards direct deposition of bioassay data. Nucleic Acids Res. 2019, 47, D930-D940. [CrossRef] [PubMed]

82. Fang, J.; Li, Y.; Liu, R.; Pang, X.; Li, C.; Yang, R.; He, Y.; Lian, W.; Liu, A.L.; Du, G.H. Discovery of multitarget-directed ligands against Alzheimer's disease through systematic prediction of chemical-protein interactions. J. Chem. Inf. Model. 2015, 55, 149-164. [CrossRef]

83. Shao, Y.M.; Ma, X.; Paira, P.; Tan, A.; Herr, D.R.; Lim, K.L.; Ng, C.H.; Venkatesan, G.; Klotz, K.N.; Federico, S.; et al. Discovery of indolylpiperazinylpyrimidines with dual-target profiles at adenosine A2A and dopamine D2 receptors for Parkinson's disease treatment. PLoS ONE 2018, 13, e0188212. [CrossRef]

84. Michielan, L.; Bolcato, C.; Federico, S.; Cacciari, B.; Bacilieri, M.; Klotz, K.N.; Kachler, S.; Pastorin, G.; Cardin, R.; Sperduti, A.; et al. Combining selectivity and affinity predictions using an integrated Support Vector Machine (SVM) approach: An alternative tool to discriminate between the human adenosine $\mathrm{A}(2 \mathrm{~A})$ and $\mathrm{A}(3)$ receptor pyrazolo-triazolo-pyrimidine antagonists binding sites. Bioorg. Med. Chem. 2009, 17, 5259-5274. [CrossRef]

85. Deshmukh, A.L.; Chandra, S.; Singh, D.K.; Siddiqi, M.I.; Banerjee, D. Identification of human flap endonuclease 1 (FEN1) inhibitors using a machine learning based consensus virtual screening. Mol. Biosyst. 2017, 13, 1630-1639. [CrossRef]

86. Zhang, H.; Liu, W.; Liu, Z.; Ju, Y.; Xu, M.; Zhang, Y.; Wu, X.; Gu, Q.; Wang, Z.; Xu, J. Discovery of indoleamine 2,3-dioxygenase inhibitors using machine learning based virtual screening. Medchemcomm 2018, 9, 937-945. [CrossRef]

87. Kang, D.; Pang, X.; Lian, W.; Xu, L.; Wang, J.; Jia, H.; Zhang, B.; Liu, A.-L.; Du, G.-H. Discovery of VEGFR2 inhibitors by integrating naïve Bayesian classification, molecular docking and drug screening approaches. RSC Adv. 2018, 8, 5286-5297. [CrossRef]

88. Montanari, F.; Cseke, A.; Wlcek, K.; Ecker, G.F. Virtual Screening of DrugBank Reveals Two Drugs as New BCRP Inhibitors. SLAS Discov. 2017, 22, 86-93. [CrossRef]

89. Allen, B.K.; Mehta, S.; Ember, S.W.; Schonbrunn, E.; Ayad, N.; Schurer, S.C. Large-Scale Computational Screening Identifies First in Class Multitarget Inhibitor of EGFR Kinase and BRD4. Sci. Rep. 2015, 5, 16924. [CrossRef] [PubMed]

90. Winkler, D.A. Use of Artificial Intelligence and Machine Learning for Discovery of Drugs for Neglected Tropical Diseases. Front. Chem. 2021, 9, 614073. [CrossRef] [PubMed]

91. Lima Guimaraes, G.; Sanchez-Lengeling, B.; Outeiral, C.; Cunha Farias, P.L.; Aspuru-Guzik, A. Objective-Reinforced Generative Adversarial Networks (ORGAN) for Sequence Generation Models. arXiv 2017, arXiv:1705.10843.

92. Keshavarzi Arshadi, A.; Salem, M.; Collins, J.; Yuan, J.S.; Chakrabarti, D. DeepMalaria: Artificial Intelligence Driven Discovery of Potent Antiplasmodials. Front. Pharm. 2019, 10, 1526. [CrossRef]

93. Aslam, B.; Wang, W.; Arshad, M.I.; Khurshid, M.; Muzammil, S.; Rasool, M.H.; Nisar, M.A.; Alvi, R.F.; Aslam, M.A.; Qamar, M.U.; et al. Antibiotic resistance: A rundown of a global crisis. Infect Drug Resist. 2018, 11, 1645-1658. [CrossRef] [PubMed]

94. Stokes, J.M.; Yang, K.; Swanson, K.; Jin, W.; Cubillos-Ruiz, A.; Donghia, N.M.; MacNair, C.R.; French, S.; Carfrae, L.A.; BloomAckermann, Z.; et al. A Deep Learning Approach to Antibiotic Discovery. Cell 2020, 180, 688-702. [CrossRef]

95. Li, L.; Le, X.; Wang, L.; Gu, Q.; Zhou, H.; Xu, J. Discovering new DNA gyrase inhibitors using machine learning approaches. RSC Adv. 2015, 5, 105600-105608. [CrossRef] 
96. Ekins, S.; Freundlich, J.S.; Clark, A.M.; Anantpadma, M.; Davey, R.A.; Madrid, P. Machine learning models identify molecules active against the Ebola virus in vitro. F1000Research 2015, 4, 1091. [CrossRef]

97. Popova, M.; Isayev, O.; Tropsha, A. Deep reinforcement learning for de novo drug design. Sci. Adv. 2018, 4, eaap7885. [CrossRef] [PubMed]

98. Gupta, A.; Muller, A.T.; Huisman, B.J.H.; Fuchs, J.A.; Schneider, P.; Schneider, G. Generative Recurrent Networks for De Novo Drug Design. Mol. Inf. 2018, 37, 1700111. [CrossRef]

99. Merk, D.; Friedrich, L.; Grisoni, F.; Schneider, G. De Novo Design of Bioactive Small Molecules by Artificial Intelligence. Mol. Inf. 2018, 37, 1700153. [CrossRef] [PubMed]

100. Zhavoronkov, A.; Ivanenkov, Y.A.; Aliper, A.; Veselov, M.S.; Aladinskiy, V.A.; Aladinskaya, A.V.; Terentiev, V.A.; Polykovskiy, D.A.; Kuznetsov, M.D.; Asadulaev, A.; et al. Deep learning enables rapid identification of potent DDR1 kinase inhibitors. Nat. Biotechnol. 2019, 37, 1038-1040. [CrossRef] [PubMed]

101. McCloskey, K.; Sigel, E.A.; Kearnes, S.; Xue, L.; Tian, X.; Moccia, D.; Gikunju, D.; Bazzaz, S.; Chan, B.; Clark, M.A.; et al. Machine Learning on DNA-Encoded Libraries: A New Paradigm for Hit Finding. J. Med. Chem. 2020, 63, 8857-8866. [CrossRef]

102. Zeng, X.; Zhu, S.; Lu, W.; Liu, Z.; Huang, J.; Zhou, Y.; Fang, J.; Huang, Y.; Guo, H.; Li, L.; et al. Target identification among known drugs by deep learning from heterogeneous networks. Chem. Sci. 2020, 11, 1775-1797. [CrossRef]

103. Dennis, M.J.; Beijnen, J.H.; Grochow, L.B.; van Warmerdam, L.J. An overview of the clinical pharmacology of topotecan. Semin. Oncol. 1997, 24, S5-S12.

104. Kollmannsberger, C.; Mross, K.; Jakob, A.; Kanz, L.; Bokemeyer, C. Topotecan-A novel topoisomerase I inhibitor: Pharmacology and clinical experience. Oncology 1999, 56, 1-12. [CrossRef]

105. White, S.C.; Cheeseman, S.; Thatcher, N.; Anderson, H.; Carrington, B.; Hearn, S.; Ross, G.; Ranson, M. Phase II study of oral topotecan in advanced non-small cell lung cancer. Clin. Cancer Res. 2000, 6, 868-873.

106. Yang, Y.; Winger, R.C.; Lee, P.W.; Nuro-Gyina, P.K.; Minc, A.; Larson, M.; Liu, Y.; Pei, W.; Rieser, E.; Racke, M.K.; et al. Impact of suppressing retinoic acid-related orphan receptor gamma $t(R O R)$ gammat in ameliorating central nervous system autoimmunity. Clin. Exp. Immunol. 2015, 179, 108-118. [CrossRef] [PubMed]

107. Zhang, Y.; Luo, X.Y.; Wu, D.H.; Xu, Y. ROR nuclear receptors: Structures, related diseases, and drug discovery. Acta Pharm. Sin. 2015, 36, 71-87. [CrossRef]

108. Madhukar, N.S.; Khade, P.K.; Huang, L.; Gayvert, K.; Galletti, G.; Stogniew, M.; Allen, J.E.; Giannakakou, P.; Elemento, O. A Bayesian machine learning approach for drug target identification using diverse data types. Nat. Commun. 2019, 10, 5221. [CrossRef] [PubMed]

109. Dezso, Z.; Ceccarelli, M. Machine learning prediction of oncology drug targets based on protein and network properties. BMC Bioinform. 2020, 21, 104. [CrossRef]

110. Terranova, N.; Venkatakrishnan, K.; Benincosa, L.J. Application of Machine Learning in Translational Medicine: Current Status and Future Opportunities. AAPS J. 2021, 23, 74. [CrossRef]

111. van der Graaf, P.H.; Benson, N. Systems pharmacology: Bridging systems biology and pharmacokinetics-pharmacodynamics (PKPD) in drug discovery and development. Pharm. Res. 2011, 28, 1460-1464. [CrossRef]

112. Stern, A.M.; Schurdak, M.E.; Bahar, I.; Berg, J.M.; Taylor, D.L. A Perspective on Implementing a Quantitative Systems Pharmacology Platform for Drug Discovery and the Advancement of Personalized Medicine. J. Biomol. Screen. 2016, 21, 521-534. [CrossRef] [PubMed]

113. Agoram, B.M.; Martin, S.W.; van der Graaf, P.H. The role of mechanism-based pharmacokinetic-pharmacodynamic (PK-PD) modelling in translational research of biologics. Drug Discov. Today 2007, 12, 1018-1024. [CrossRef] [PubMed]

114. Bradshaw, E.L.; Spilker, M.E.; Zang, R.; Bansal, L.; He, H.; Jones, R.D.O.; Le, K.; Penney, M.; Schuck, E.; Topp, B.; et al. Applications of Quantitative Systems Pharmacology in Model-Informed Drug Discovery: Perspective on Impact and Opportunities. CPT Pharmacomet. Syst. Pharm. 2019, 8, 777-791. [CrossRef]

115. Lazarou, G.; Chelliah, V.; Small, B.G.; Walker, M.; van der Graaf, P.H.; Kierzek, A.M. Integration of Omics Data Sources to Inform Mechanistic Modeling of Immune-Oncology Therapies: A Tutorial for Clinical Pharmacologists. Clin. Pharm. 2020, 107, 858-870. [CrossRef]

116. Ramm, S.; Todorov, P.; Chandrasekaran, V.; Dohlman, A.; Monteiro, M.B.; Pavkovic, M.; Muhlich, J.; Shankaran, H.; Chen, W.W.; Mettetal, J.T.; et al. A Systems Toxicology Approach for the Prediction of Kidney Toxicity and Its Mechanisms In Vitro. Toxicol. Sci. 2019, 169, 54-69. [CrossRef]

117. Song, J.; Wang, L.; Ng, N.N.; Zhao, M.; Shi, J.; Wu, N.; Li, W.; Liu, Z.; Yeom, K.W.; Tian, J. Development and Validation of a Machine Learning Model to Explore Tyrosine Kinase Inhibitor Response in Patients With Stage IV EGFR Variant-Positive Non-Small Cell Lung Cancer. JAMA Netw. Open 2020, 3, e2030442. [CrossRef]

118. Lu, J.; Bender, B.; Jin, J.Y.; Guan, Y. Deep learning prediction of patient response time course from early data via neuralpharmacokinetic/pharmacodynamic modelling. Nat. Mach. Intell. 2021, 3, 696-704. [CrossRef]

119. Obermeyer, Z.; Emanuel, E.J. Predicting the Future-Big Data, Machine Learning, and Clinical Medicine. N. Engl. J. Med. 2016, 375, 1216-1219. [CrossRef]

120. Aggarwal, R.; Sounderajah, V.; Martin, G.; Ting, D.S.W.; Karthikesalingam, A.; King, D.; Ashrafian, H.; Darzi, A. Diagnostic accuracy of deep learning in medical imaging: A systematic review and meta-analysis. NPJ Digit. Med. 2021, 4, 65. [CrossRef] [PubMed]

121. Briganti, G.; Le Moine, O. Artificial Intelligence in Medicine: Today and Tomorrow. Front. Med. 2020, 7, 27. [CrossRef] [PubMed] 
122. Nichols, J.A.; Herbert Chan, H.W.; Baker, M.A.B. Machine learning: Applications of artificial intelligence to imaging and diagnosis. Biophys. Rev. 2019, 11, 111-118. [CrossRef]

123. Litjens, G.; Kooi, T.; Bejnordi, B.E.; Setio, A.A.A.; Ciompi, F.; Ghafoorian, M.; van der Laak, J.; van Ginneken, B.; Sanchez, C.I. A survey on deep learning in medical image analysis. Med. Image Anal. 2017, 42, 60-88. [CrossRef]

124. Benjamens, S.; Dhunnoo, P.; Mesko, B. The state of artificial intelligence-based FDA-approved medical devices and algorithms: An online database. NPJ Digit. Med. 2020, 3, 118. [CrossRef]

125. Topol, E.J. High-performance medicine: The convergence of human and artificial intelligence. Nat. Med. 2019, 25, 44-56. [CrossRef]

126. Muehlematter, U.J.; Daniore, P.; Vokinger, K.N. Approval of artificial intelligence and machine learning-based medical devices in the USA and Europe (2015-2020): A comparative analysis. Lancet Digit. Health 2021, 3, e195-e203. [CrossRef]

127. Chartrand, G.; Cheng, P.M.; Vorontsov, E.; Drozdzal, M.; Turcotte, S.; Pal, C.J.; Kadoury, S.; Tang, A. Deep Learning: A Primer for Radiologists. Radiographics 2017, 37, 2113-2131. [CrossRef] [PubMed]

128. Adadi, A.; Adadi, S.; Berrada, M. Gastroenterology Meets Machine Learning: Status Quo and Quo Vadis. Adv. Bioinform. 2019, 2019, 1870975. [CrossRef]

129. van der Sommen, F.; de Groof, J.; Struyvenberg, M.; van der Putten, J.; Boers, T.; Fockens, K.; Schoon, E.J.; Curvers, W.; de With, P.; Mori, Y.; et al. Machine learning in GI endoscopy: Practical guidance in how to interpret a novel field. Gut 2020, 69, 2035-2045. [CrossRef] [PubMed]

130. Auger, S.D.; Jacobs, B.M.; Dobson, R.; Marshall, C.R.; Noyce, A.J. Big data, machine learning and artificial intelligence: A neurologist's guide. Pract. Neurol. 2020, 21, 4-11. [CrossRef] [PubMed]

131. Zhu, G.; Jiang, B.; Tong, L.; Xie, Y.; Zaharchuk, G.; Wintermark, M. Applications of Deep Learning to Neuro-Imaging Techniques. Front. Neurol. 2019, 10, 869. [CrossRef]

132. Tong, Y.; Lu, W.; Yu, Y.; Shen, Y. Application of machine learning in ophthalmic imaging modalities. Eye Vis. 2020, 7, 22. [CrossRef]

133. Ting, D.S.W.; Pasquale, L.R.; Peng, L.; Campbell, J.P.; Lee, A.Y.; Raman, R.; Tan, G.S.W.; Schmetterer, L.; Keane, P.A.; Wong, T.Y. Artificial intelligence and deep learning in ophthalmology. Br. J. Ophthalmol. 2019, 103, 167-175. [CrossRef]

134. Quer, G.; Arnaout, R.; Henne, M.; Arnaout, R. Machine Learning and the Future of Cardiovascular Care: JACC State-of-the-Art Review. J. Am. Coll. Cardiol. 2021, 77, 300-313. [CrossRef] [PubMed]

135. Cuocolo, R.; Perillo, T.; De Rosa, E.; Ugga, L.; Petretta, M. Current applications of big data and machine learning in cardiology. J. Geriatr. Cardiol. 2019, 16, 601-607. [CrossRef]

136. Chan, S.; Reddy, V.; Myers, B.; Thibodeaux, Q.; Brownstone, N.; Liao, W. Machine Learning in Dermatology: Current Applications, Opportunities, and Limitations. Dermatol. Ther. 2020, 10, 365-386. [CrossRef] [PubMed]

137. Serag, A.; Ion-Margineanu, A.; Qureshi, H.; McMillan, R.; Saint Martin, M.J.; Diamond, J.; O’Reilly, P.; Hamilton, P. Translational AI and Deep Learning in Diagnostic Pathology. Front. Med. 2019, 6, 185. [CrossRef] [PubMed]

138. Hamamoto, R.; Suvarna, K.; Yamada, M.; Kobayashi, K.; Shinkai, N.; Miyake, M.; Takahashi, M.; Jinnai, S.; Shimoyama, R.; Sakai, A.; et al. Application of Artificial Intelligence Technology in Oncology: Towards the Establishment of Precision Medicine. Cancers 2020, 12, 3532. [CrossRef]

139. Davenport, T.; Kalakota, R. The potential for artificial intelligence in healthcare. Future Healthc. J. 2019, 6, 94-98. [CrossRef] [PubMed]

140. Cutillo, C.M.; Sharma, K.R.; Foschini, L.; Kundu, S.; Mackintosh, M.; Mandl, K.D.; MI in Healthcare Workshop Working Group. Machine intelligence in healthcare-perspectives on trustworthiness, explainability, usability, and transparency. NPJ Digit. Med. 2020, 3, 47. [CrossRef] [PubMed]

141. Shah, P.; Kendall, F.; Khozin, S.; Goosen, R.; Hu, J.; Laramie, J.; Ringel, M.; Schork, N. Artificial intelligence and machine learning in clinical development: A translational perspective. NPJ Digit. Med. 2019, 2, 69. [CrossRef] [PubMed]

142. Adlung, L.; Cohen, Y.; Mor, U.; Elinav, E. Machine learning in clinical decision making. Med 2021, 2, 642-665. [CrossRef]

143. Komatsu, M.; Sakai, A.; Dozen, A.; Shozu, K.; Yasutomi, S.; Machino, H.; Asada, K.; Kaneko, S.; Hamamoto, R. Towards Clinical Application of Artificial Intelligence in Ultrasound Imaging. Biomedicines 2021, 9, 720. [CrossRef]

144. Khemasuwan, D.; Sorensen, J.S.; Colt, H.G. Artificial intelligence in pulmonary medicine: Computer vision, predictive model and COVID-19. Eur. Respir. Rev. 2020, 29, 200181. [CrossRef]

145. Kaplan, A.; Cao, H.; FitzGerald, J.M.; Iannotti, N.; Yang, E.; Kocks, J.W.H.; Kostikas, K.; Price, D.; Reddel, H.K.; Tsiligianni, I.; et al. Artificial Intelligence/Machine Learning in Respiratory Medicine and Potential Role in Asthma and COPD Diagnosis. J. Allergy Clin. Immunol. Pr. 2021, 9, 2255-2261. [CrossRef]

146. Available online: https://www.maxq.ai/products (accessed on 14 October 2021).

147. Available online: https://global.medical.canon/products/computed-tomography/aice (accessed on 14 October 2021).

148. Available online: https://www.siemens-healthineers.com/en-us/digital-health-solutions/digital-solutions-overview/clinicaldecision-support/ai-rad-companion (accessed on 14 October 2021).

149. Chamberlin, J.; Kocher, M.R.; Waltz, J.; Snoddy, M.; Stringer, N.F.C.; Stephenson, J.; Sahbaee, P.; Sharma, P.; Rapaka, S.; Schoepf, U.J.; et al. Automated detection of lung nodules and coronary artery calcium using artificial intelligence on low-dose CT scans for lung cancer screening: Accuracy and prognostic value. BMC Med. 2021, 19, 55. [CrossRef]

150. Available online: https://www.varian.com/fi/resources-support/events/virtual-2020/artificial-intelligence-ai (accessed on 14 October 2021).

151. Available online: https://www.amcad.com.tw/product/uo (accessed on 14 October 2021).

152. Available online: https://www.amcad.com.tw/product/future_detail/3 (accessed on 14 October 2021). 
153. Available online: https://www.amcad.com.tw/product/ut (accessed on 14 October 2021).

154. Li, T.; Jiang, Z.; Lu, M.; Zou, S.; Wu, M.; Wei, T.; Wang, L.; Li, J.; Hu, Z.; Cheng, X.; et al. Computer-aided diagnosis system of thyroid nodules ultrasonography: Diagnostic performance difference between computer-aided diagnosis and 111 radiologists. Medicine 2020, 99, e20634. [CrossRef] [PubMed]

155. Available online: https://www.amcad.com.tw/product/future_detail/2 (accessed on 14 October 2021).

156. Available online: https://www.arterys.com/clinicalapp/cardioapp (accessed on 14 October 2021).

157. Available online: https://www.arterys.com/clinicalapp/lungapp (accessed on 14 October 2021).

158. Available online: https:/ / www.arterys.com/ (accessed on 14 October 2021).

159. Available online: https://www.verathon.com/bladderscan-prime-plus/ (accessed on 14 October 2021).

160. Available online: https://www.gehealthcare.co.uk/products/computed-tomography/advanced-visualization/bone-vcar (accessed on 14 October 2021).

161. Available online: https://www.brainomix.com/e-cta/ (accessed on 14 October 2021).

162. Zelenak, K.; Krajina, A.; Meyer, L.; Fiehler, J.; Esmint Artificial, I.; Robotics Ad Hoc, C.; Behme, D.; Bulja, D.; Caroff, J.; Chotai, A.A.; et al. How to Improve the Management of Acute Ischemic Stroke by Modern Technologies, Artificial Intelligence, and New Treatment Methods. Life 2021, 11, 488. [CrossRef] [PubMed]

163. Available online: https:/ / www.aidoc.com/ (accessed on 14 October 2021).

164. Available online: https://www.circlecvi.com/cvi42/cardiac-mri/deep-learning/ (accessed on 14 October 2021).

165. Karimi-Bidhendi, S.; Arafati, A.; Cheng, A.L.; Wu, Y.; Kheradvar, A.; Jafarkhani, H. Fully-automated deep-learning segmentation of pediatric cardiovascular magnetic resonance of patients with complex congenital heart diseases. J. Cardiovasc. Magn. Reson. 2020, 22, 80. [CrossRef] [PubMed]

166. Available online: https://www.claripi.com/clarict-ai/ (accessed on 14 October 2021).

167. Available online: https://www.riveraintech.com/clearread-ai-solutions/clearread-ct/ (accessed on 14 October 2021).

168. Wagner, A.K.; Hapich, A.; Psychogios, M.N.; Teichgraber, U.; Malich, A.; Papageorgiou, I. Computer-Aided Detection of Pulmonary Nodules in Computed Tomography Using ClearReadCT. J. Med. Syst. 2019, 43, 58. [CrossRef]

169. Available online: https:// curemetrix.com/cm-triage-2/ (accessed on 14 October 2021).

170. Available online: https://www.viz.ai/ (accessed on 14 October 2021).

171. Available online: https://www.gehealthcare.co.uk/products/radiography-systems/mobile-xray-systems/critical-care-suiteon-optima-xr240amx (accessed on 14 October 2021).

172. Available online: https://www.keyamedical.com/curarad-ich/ (accessed on 14 October 2021).

173. Available online: https://www.gehealthcare.co.uk/ (accessed on 14 October 2021).

174. Available online: https://www.deep-voxel.com/\#/home (accessed on 14 October 2021).

175. Available online: https:// captionhealth.com/ (accessed on 14 October 2021).

176. Available online: https:/ / ferriscan.com/ferrismart/ (accessed on 14 October 2021).

177. Available online: https://www.resonancehealth.com/products/ferriscan-mri-measurement-of-liver-iron-concentration.html (accessed on 14 October 2021).

178. Padeniya, P.; Siriwardana, S.; Ediriweera, D.; Samarasinghe, N.; Silva, S.; Silva, I.; Ahamed, N.; Niriella, M.; Premawardhena, A. Comparison of liver MRI R2(FerriScan(R)) VS liver MRI T2* as a measure of body iron load in a cohort of beta thalassaemia major patients. Orphanet J. Rare Dis. 2020, 15, 26. [CrossRef] [PubMed]

179. Available online: https://www.zebra-med.com/solutions/triage/healthcxr (accessed on 14 October 2021).

180. Available online: https:/ / www.zebra-med.com/ (accessed on 14 October 2021).

181. Available online: https://icometrix.com/services/icobrain-ms (accessed on 14 October 2021).

182. Struyfs, H.; Sima, D.M.; Wittens, M.; Ribbens, A.; Pedrosa de Barros, N.; Phan, T.V.; Ferraz Meyer, M.I.; Claes, L.; Niemantsverdriet, E.; Engelborghs, S.; et al. Automated MRI volumetry as a diagnostic tool for Alzheimer's disease: Validation of icobrain dm. Neuroimage Clin. 2020, 26, 102243. [CrossRef]

183. Available online: https://www.usa.philips.com/healthcare/solutions/diagnostic-informatics (accessed on 14 October 2021).

184. Available online: https://global.infervision.com/product/5/ (accessed on 14 October 2021).

185. Available online: https://clinicaltrials.gov/ct2/show/NCT04119960 (accessed on 14 October 2021).

186. Available online: https://www.infinittna.com/solutions/radiology/infinitt-pacs/ (accessed on 14 October 2021).

187. Available online: https://www.imagebiopsy.com/product/koala-ce (accessed on 14 October 2021).

188. Available online: https:/ / koiosmedical.com/ (accessed on 14 October 2021).

189. Available online: https:// perspectum.com/products/livermultiscan (accessed on 14 October 2021).

190. Available online: https://www.dia-analysis.com/lvivo-ef-app (accessed on 14 October 2021).

191. Available online: https://thirona.eu/solutions/chestct/ (accessed on 14 October 2021).

192. Available online: https://perspectum.com/products/mrcp (accessed on 14 October 2021).

193. Available online: https://www.philips.com.au/healthcare/product/HCNMRF320/mrcat-brain-mr-rt-clinical-application (accessed on 14 October 2021).

194. Available online: https:/ /imagen.ai/ (accessed on 14 October 2021).

195. Available online: https://www.algomedica.com/low-radation-ct-scans-algomedica (accessed on 14 October 2021).

196. Available online: https://www.icadmed.com/powerlook-density-assessment.html (accessed on 14 October 2021). 
197. Available online: https://www.icadmed.com/profoundai.html (accessed on 14 October 2021).

198. Available online: https://www.qlarityimaging.com/ (accessed on 14 October 2021).

199. Available online: https:/ / quibim.com/ (accessed on 14 October 2021).

200. Available online: https:/ / www.rapidai.com/rapid-aspects (accessed on 14 October 2021).

201. Available online: https:/ / www.rapidai.com/rapid-ich (accessed on 14 October 2021).

202. Available online: https://www.raysearchlabs.com/data-handling-and-machine-learning/ (accessed on 14 October 2021).

203. Available online: https://www.raysearchlabs.com/machine-learning-in-raystation/ (accessed on 14 October 2021).

204. Available online: https:/ / www.radiobotics.com/products (accessed on 14 October 2021).

205. Available online: https:/ / behold.ai/ (accessed on 14 October 2021).

206. Available online: https://www.imagingbiometrics.com/what-we-offer/product-services/ib-stonechecker/ (accessed on 14 October 2021).

207. Available online: https:/ / strokeviewer.nico-lab.com/ (accessed on 14 October 2021).

208. Available online: https:/ / subtlemedical.com/subtlemr/ (accessed on 14 October 2021).

209. Available online: https://subtlemedical.com/subtlepet/ (accessed on 14 October 2021).

210. Available online: https://www.siemens-healthineers.com/computed-tomography/options-upgrades/clinical-applications / syngo-ct-cardiac-function (accessed on 14 October 2021).

211. Available online: https://screenpoint-medical.com/fusion-ai/ (accessed on 14 October 2021).

212. Sasaki, M.; Tozaki, M.; Rodriguez-Ruiz, A.; Yotsumoto, D.; Ichiki, Y.; Terawaki, A.; Oosako, S.; Sagara, Y.; Sagara, Y. Artificial intelligence for breast cancer detection in mammography: Experience of use of the ScreenPoint Medical Transpara system in 310 Japanese women. Breast Cancer 2020, 27, 642-651. [CrossRef]

213. Available online: https://www.veolity.com/ (accessed on 14 October 2021).

214. Available online: https://mirada-medical.com/ (accessed on 14 October 2021).

215. Available online: https:/ / www.carewell.com.cn/en/AI-ECG.html (accessed on 14 October 2021).

216. Available online: https://www.carewellhealth.com/products_aiecg.html (accessed on 14 October 2021).

217. Available online: https:/ / www.biotricity.com/bioflux-2/ (accessed on 14 October 2021).

218. Available online: https://www.ultromics.com/echogo (accessed on 14 October 2021).

219. Available online: https://www.ekohealth.com/ (accessed on 14 October 2021).

220. Available online: https:/ / emurmur.com/ai/ (accessed on 14 October 2021).

221. Available online: https://www.kardia.com/ (accessed on 14 October 2021).

222. Available online: https://kosmosplatform.com/ (accessed on 14 October 2021).

223. Available online: https://www.ventripoint.com/explore-vms-3-0 (accessed on 14 October 2021).

224. Available online: https://altoida.com/ (accessed on 14 October 2021).

225. Meier, I.B.; Buegler, M.; Harms, R.; Seixas, A.; Coltekin, A.; Tarnanas, I. Using a Digital Neuro Signature to measure longitudinal individual-level change in Alzheimer's disease: The Altoida large cohort study. NPJ Digit. Med. 2021, 4, 101. [CrossRef]

226. Available online: https:/ /www.brainscope.com/ (accessed on 14 October 2021).

227. Available online: https:/ / cognoa.com/providers/ (accessed on 14 October 2021).

228. Available online: https:// coaptengineering.com/user-manual/complete-control-room (accessed on 14 October 2021).

229. Available online: https:/ / www.ensodata.com/ensosleep/ (accessed on 14 October 2021).

230. Available online: https:/ / www.qbtech.com/adhd-tests\# (accessed on 14 October 2021).

231. Hult, N.; Kadesjo, J.; Kadesjo, B.; Gillberg, C.; Billstedt, E. ADHD and the QbTest: Diagnostic Validity of QbTest. J. Atten. Disord. 2018, 22, 1074-1080. [CrossRef]

232. Available online: https://www.zeiss.com/meditec/int/product-portfolio/retinal-cameras / zeiss-clarus-700.html (accessed on 14 October 2021).

233. Available online: https://www.eyenuk.com/en/products/eyeart/ (accessed on 14 October 2021).

234. Ipp, E.L.I.; Shah, V.N.; Bode, B.W.; Sadda, S.R. 599-P: Diabetic Retinopathy (DR) Screening Performance of General Ophthalmologists, Retina Specialists, and Artificial Intelligence (AI): Analysis from a Pivotal Multicenter Prospective Clinical Trial. Diabetes 2019, 68, 599. [CrossRef]

235. Available online: https://www.digitaldiagnostics.com/ (accessed on 14 October 2021).

236. Abramoff, M.D.; Lou, Y.; Erginay, A.; Clarida, W.; Amelon, R.; Folk, J.C.; Niemeijer, M. Improved Automated Detection of Diabetic Retinopathy on a Publicly Available Dataset Through Integration of Deep Learning. Invest. Ophthalmol. Vis. Sci. 2016, 57, 5200-5206. [CrossRef]

237. Available online: https://dreamed-diabetes.com/advisor/ (accessed on 14 October 2021).

238. Available online: https://www.medtronicdiabetes.com/products/guardian-connect-continuous-glucose-monitoring-system (accessed on 14 October 2021).

239. Available online: https:/ / cleverculturesystems.com/apas-independence/ (accessed on 14 October 2021).

240. Brenton, L.; Waters, M.J.; Stanford, T.; Giglio, S. Clinical evaluation of the APAS(R) Independence: Automated imaging and interpretation of urine cultures using artificial intelligence with composite reference standard discrepant resolution. J. Microbiol. Methods 2020, 177, 106047. [CrossRef]

241. Available online: https:/ / nightowl.care/ (accessed on 14 October 2021). 
242. Available online: https://www.nuvasive.com/surgical-solutions/pulse/ (accessed on 14 October 2021).

243. Available online: https://www.sightdx.com/product (accessed on 14 October 2021).

244. Bachar, N.; Benbassat, D.; Brailovsky, D.; Eshel, Y.; Gluck, D.; Levner, D.; Levy, S.; Pecker, S.; Yurkovsky, E.; Zait, A.; et al. An artificial intelligence-assisted diagnostic platform for rapid near-patient hematology. Am. J. Hematol. 2021, 96, 1264-1274. [CrossRef]

245. Available online: https://www.impedimed.com/sozo-launched-first-product-for-health-and-wellness-markets / (accessed on 14 October 2021).

246. Available online: https:/ / respiri.co/products/ (accessed on 14 October 2021).

247. Yu, K.H.; Zhang, C.; Berry, G.J.; Altman, R.B.; Re, C.; Rubin, D.L.; Snyder, M. Predicting non-small cell lung cancer prognosis by fully automated microscopic pathology image features. Nat. Commun. 2016, 7, 12474. [CrossRef] [PubMed]

248. Ozaki, Y.; Yamada, H.; Kikuchi, H.; Hirotsu, A.; Murakami, T.; Matsumoto, T.; Kawabata, T.; Hiramatsu, Y.; Kamiya, K.; Yamauchi, T.; et al. Label-free classification of cells based on supervised machine learning of subcellular structures. PLoS ONE 2019, 14, e0211347. [CrossRef] [PubMed]

249. Simm, J.; Klambauer, G.; Arany, A.; Steijaert, M.; Wegner, J.K.; Gustin, E.; Chupakhin, V.; Chong, Y.T.; Vialard, J.; Buijnsters, P.; et al. Repurposing High-Throughput Image Assays Enables Biological Activity Prediction for Drug Discovery. Cell Chem. Biol. 2018, 25, 611-618. [CrossRef] [PubMed]

250. Nassar, M.; Doan, M.; Filby, A.; Wolkenhauer, O.; Fogg, D.K.; Piasecka, J.; Thornton, C.A.; Carpenter, A.E.; Summers, H.D.; Rees, P.; et al. Label-Free Identification of White Blood Cells Using Machine Learning. Cytometry Part A 2019, 95, 836-842. [CrossRef]

251. Coudray, N.; Ocampo, P.S.; Sakellaropoulos, T.; Narula, N.; Snuderl, M.; Fenyo, D.; Moreira, A.L.; Razavian, N.; Tsirigos, A. Classification and mutation prediction from non-small cell lung cancer histopathology images using deep learning. Nat. Med. 2018, 24, 1559-1567. [CrossRef]

252. Chang, C.H.; Lin, C.H.; Lane, H.Y. Machine Learning and Novel Biomarkers for the Diagnosis of Alzheimer's Disease. Int. J. Mol. Sci. 2021, 22, 2761. [CrossRef]

253. Kang, C.; Wang, D.; Zhang, X.; Wang, L.; Wang, F.; Chen, J. Construction and Validation of a Lung Cancer Diagnostic Model Based on 6-Gene Methylation Frequency in Blood, Clinical Features, and Serum Tumor Markers. Comput. Math. Methods Med. 2021, 2021, 9987067. [CrossRef]

254. Brown, J.M.; Campbell, J.P.; Beers, A.; Chang, K.; Ostmo, S.; Chan, R.V.P.; Dy, J.; Erdogmus, D.; Ioannidis, S.; Kalpathy-Cramer, J.; et al. Automated Diagnosis of Plus Disease in Retinopathy of Prematurity Using Deep Convolutional Neural Networks. JAMA Ophthalmol. 2018, 136, 803-810. [CrossRef] [PubMed]

255. Dai, L.; Wu, L.; Li, H.; Cai, C.; Wu, Q.; Kong, H.; Liu, R.; Wang, X.; Hou, X.; Liu, Y.; et al. A deep learning system for detecting diabetic retinopathy across the disease spectrum. Nat. Commun. 2021, 12, 3242. [CrossRef]

256. Gargeya, R.; Leng, T. Automated Identification of Diabetic Retinopathy Using Deep Learning. Ophthalmology 2017, 124, 962-969. [CrossRef] [PubMed]

257. Ariya, A.; Asha, S. Automated detection of macular Edema using machine learning algorithm. AIP Conf. Proc. 2020, 2222, 030015. [CrossRef]

258. Zhang, Q.; Liu, Z.; Li, J.; Liu, G. Identifying Diabetic Macular Edema and Other Retinal Diseases by Optical Coherence Tomography Image and Multiscale Deep Learning. Diabetes Metab. Syndr. Obes. 2020, 13, 4787-4800. [CrossRef] [PubMed]

259. Kuwayama, S.; Ayatsuka, Y.; Yanagisono, D.; Uta, T.; Usui, H.; Kato, A.; Takase, N.; Ogura, Y.; Yasukawa, T. Automated Detection of Macular Diseases by Optical Coherence Tomography and Artificial Intelligence Machine Learning of Optical Coherence Tomography Images. J. Ophthalmol. 2019, 2019, 6319581. [CrossRef]

260. Burlina, P.M.; Joshi, N.; Pekala, M.; Pacheco, K.D.; Freund, D.E.; Bressler, N.M. Automated Grading of Age-Related Macular Degeneration From Color Fundus Images Using Deep Convolutional Neural Networks. JAMA Ophthalmol. 2017, 135, 1170-1176. [CrossRef]

261. Li, Z.; He, Y.; Keel, S.; Meng, W.; Chang, R.T.; He, M. Efficacy of a Deep Learning System for Detecting Glaucomatous Optic Neuropathy Based on Color Fundus Photographs. Ophthalmology 2018, 125, 1199-1206. [CrossRef]

262. Ran, A.R.; Tham, C.C.; Chan, P.P.; Cheng, C.Y.; Tham, Y.C.; Rim, T.H.; Cheung, C.Y. Deep learning in glaucoma with optical coherence tomography: A review. Eye 2021, 35, 188-201. [CrossRef]

263. Asaoka, R.; Murata, H.; Hirasawa, K.; Fujino, Y.; Matsuura, M.; Miki, A.; Kanamoto, T.; Ikeda, Y.; Mori, K.; Iwase, A.; et al. Using Deep Learning and Transfer Learning to Accurately Diagnose Early-Onset Glaucoma From Macular Optical Coherence Tomography Images. Am. J. Ophthalmol. 2019, 198, 136-145. [CrossRef]

264. Kamal, H.; Lopez, V.; Sheth, S.A. Machine Learning in Acute Ischemic Stroke Neuroimaging. Front. Neurol. 2018, 9, 945. [CrossRef]

265. Plis, S.M.; Hjelm, D.R.; Salakhutdinov, R.; Allen, E.A.; Bockholt, H.J.; Long, J.D.; Johnson, H.J.; Paulsen, J.S.; Turner, J.A.; Calhoun, V.D. Deep learning for neuroimaging: A validation study. Front. Neurosci. 2014, 8, 229. [CrossRef]

266. Myszczynska, M.A.; Ojamies, P.N.; Lacoste, A.M.B.; Neil, D.; Saffari, A.; Mead, R.; Hautbergue, G.M.; Holbrook, J.D.; Ferraiuolo, L. Applications of machine learning to diagnosis and treatment of neurodegenerative diseases. Nat. Rev. Neurol. 2020, 16, 440-456. [CrossRef] [PubMed]

267. Siddiqui, M.K.; Morales-Menendez, R.; Huang, X.; Hussain, N. A review of epileptic seizure detection using machine learning classifiers. Brain Inform. 2020, 7, 1-18. [CrossRef] 
268. Abdelhameed, A.; Bayoumi, M. A Deep Learning Approach for Automatic Seizure Detection in Children With Epilepsy. Front. Comput. Neurosci. 2021, 15, 650050. [CrossRef]

269. Abbasi, B.; Goldenholz, D.M. Machine learning applications in epilepsy. Epilepsia 2019, 60, 2037-2047. [CrossRef]

270. Tenev, A.; Markovska-Simoska, S.; Kocarev, L.; Pop-Jordanov, J.; Muller, A.; Candrian, G. Machine learning approach for classification of ADHD adults. Int. J. Psychophysiol. 2014, 93, 162-166. [CrossRef]

271. Slobodin, O.; Yahav, I.; Berger, I. A Machine-Based Prediction Model of ADHD Using CPT Data. Front. Hum. Neurosci. 2020, 14, 560021. [CrossRef] [PubMed]

272. Kautzky, A.; Vanicek, T.; Philippe, C.; Kranz, G.S.; Wadsak, W.; Mitterhauser, M.; Hartmann, A.; Hahn, A.; Hacker, M.; Rujescu, D.; et al. Machine learning classification of ADHD and HC by multimodal serotonergic data. Transl. Psychiatry 2020, 10, 104. [CrossRef] [PubMed]

273. Dubreuil-Vall, L.; Ruffini, G.; Camprodon, J.A. Deep Learning Convolutional Neural Networks Discriminate Adult ADHD From Healthy Individuals on the Basis of Event-Related Spectral EEG. Front. Neurosci. 2020, 14, 251. [CrossRef] [PubMed]

274. Lu, D.; Popuri, K.; Ding, G.W.; Balachandar, R.; Beg, M.F.; Alzheimer's Disease Neuroimaging Initiative. Multimodal and Multiscale Deep Neural Networks for the Early Diagnosis of Alzheimer's Disease using structural MR and FDG-PET images. Sci. Rep. 2018, 8, 5697. [CrossRef] [PubMed]

275. Shi, J.; Zheng, X.; Li, Y.; Zhang, Q.; Ying, S. Multimodal Neuroimaging Feature Learning With Multimodal Stacked Deep Polynomial Networks for Diagnosis of Alzheimer's Disease. IEEE J. Biomed. Health Inf. 2018, 22, 173-183. [CrossRef] [PubMed]

276. Gao, X.W.; Hui, R. A deep learning based approach to classification of CT brain images. In Proceedings of the 2016 SAI Computing Conference (SAI), London, UK, 13-15 July 2016; pp. 28-31. [CrossRef]

277. Liu, L.; Zhao, S.; Chen, H.; Wang, A. A new machine learning method for identifying Alzheimer's disease. Simul. Model. Pract. Theory 2020, 99, 102023. [CrossRef]

278. Grassi, M.; Perna, G.; Caldirola, D.; Schruers, K.; Duara, R.; Loewenstein, D.A. A Clinically-Translatable Machine Learning Algorithm for the Prediction of Alzheimer's Disease Conversion in Individuals with Mild and Premild Cognitive Impairment. J. Alzheimers Dis. 2018, 61, 1555-1573. [CrossRef] [PubMed]

279. Landolfi, A.; Ricciardi, C.; Donisi, L.; Cesarelli, G.; Troisi, J.; Vitale, C.; Barone, P.; Amboni, M. Machine Learning Approaches in Parkinson's Disease. Curr. Med. Chem. 2021, 28, 6548-6568. [CrossRef]

280. Ahmadi Rastegar, D.; Ho, N.; Halliday, G.M.; Dzamko, N. Parkinson's progression prediction using machine learning and serum cytokines. NPJ Parkinsons Dis. 2019, 5, 14. [CrossRef]

281. Shu, Z.Y.; Cui, S.J.; Wu, X.; Xu, Y.; Huang, P.; Pang, P.P.; Zhang, M. Predicting the progression of Parkinson's disease using conventional MRI and machine learning: An application of radiomic biomarkers in whole-brain white matter. Magn. Reson. Med. 2021, 85, 1611-1624. [CrossRef] [PubMed]

282. Juutinen, M.; Wang, C.; Zhu, J.; Haladjian, J.; Ruokolainen, J.; Puustinen, J.; Vehkaoja, A. Parkinson's disease detection from 20-step walking tests using inertial sensors of a smartphone: Machine learning approach based on an observational case-control study. PLoS ONE 2020, 15, e0236258. [CrossRef]

283. Severson, K.A.; Chahine, L.M.; Smolensky, L.A.; Dhuliawala, M.; Frasier, M.; Ng, K.; Ghosh, S.; Hu, J. Discovery of Parkinson's disease states and disease progression modelling: A longitudinal data study using machine learning. Lancet Digit. Health 2021, 3 , e555-e564. [CrossRef]

284. Park, K.W.; Lee, E.J.; Lee, J.S.; Jeong, J.; Choi, N.; Jo, S.; Jung, M.; Do, J.Y.; Kang, D.W.; Lee, J.G.; et al. Machine Learning-Based Automatic Rating for Cardinal Symptoms of Parkinson Disease. Neurology 2021, 96, e1761-e1769. [CrossRef]

285. Mei, J.; Desrosiers, C.; Frasnelli, J. Machine Learning for the Diagnosis of Parkinson's Disease: A Review of Literature. Front. Aging Neurosci. 2021, 13, 633752. [CrossRef]

286. Maitín, A.M.; García-Tejedor, A.J.; Muñoz, J.P.R. Machine Learning Approaches for Detecting Parkinson's Disease from EEG Analysis: A Systematic Review. Appl. Sci. 2020, 10, 8662. [CrossRef]

287. Sriram, T.V.S.; Rao, M.V.; Narayana, G.V.S.; Kaladhar, D.S.V.G.K. Diagnosis of Parkinson Disease Using Machine Learning and Data Mining Systems from Voice Dataset. Adv. Intell. Syst. Comput. 2015, 327, 151-157. [CrossRef]

288. Karapinar Senturk, Z. Early diagnosis of Parkinson's disease using machine learning algorithms. Med. Hypotheses 2020, 138, 109603. [CrossRef]

289. Seetharam, K.; Shrestha, S.; Sengupta, P.P. Artificial Intelligence in Cardiac Imaging. US Cardiol. Rev. 2020, 13, 110-116. [CrossRef]

290. Constantinides, P.; Fitzmaurice, D.A. Artificial intelligence in cardiology: Applications, benefits and challenges. Br. J. Cardiol. 2018, 25, 86-87. [CrossRef]

291. Seetharam, K.; Shrestha, S.; Sengupta, P.P. Artificial Intelligence in Cardiovascular Medicine. Curr. Treat. Options Cardiovasc. Med. 2019, 21, 25. [CrossRef]

292. Sevakula, R.K.; Au-Yeung, W.M.; Singh, J.P.; Heist, E.K.; Isselbacher, E.M.; Armoundas, A.A. State-of-the-Art Machine Learning Techniques Aiming to Improve Patient Outcomes Pertaining to the Cardiovascular System. J. Am. Heart Assoc. 2020, 9, e013924. [CrossRef] [PubMed]

293. Shrestha, S.; Sengupta, P.P. The Mechanics of Machine Learning: From a Concept to Value. J. Am. Soc. Echocardiogr. 2018, 31, 1285-1287. [CrossRef] [PubMed]

294. Madani, A.; Arnaout, R.; Mofrad, M.; Arnaout, R. Fast and accurate view classification of echocardiograms using deep learning. NPJ Digit. Med. 2018, 1, 1-8. [CrossRef] 
295. Madani, A.; Ong, J.R.; Tibrewal, A.; Mofrad, M.R.K. Deep echocardiography: Data-efficient supervised and semi-supervised deep learning towards automated diagnosis of cardiac disease. NPJ Digit. Med. 2018, 1, 59. [CrossRef] [PubMed]

296. Zhang, J.; Gajjala, S.; Agrawal, P.; Tison, G.H.; Hallock, L.A.; Beussink-Nelson, L.; Lassen, M.H.; Fan, E.; Aras, M.A.; Jordan, C.; et al. Fully Automated Echocardiogram Interpretation in Clinical Practice. Circulation 2018, 138, 1623-1635. [CrossRef] [PubMed]

297. Samad, M.D.; Ulloa, A.; Wehner, G.J.; Jing, L.; Hartzel, D.; Good, C.W.; Williams, B.A.; Haggerty, C.M.; Fornwalt, B.K. Predicting Survival From Large Echocardiography and Electronic Health Record Datasets: Optimization With Machine Learning. JACC Cardiovasc. Imaging 2019, 12, 681-689. [CrossRef]

298. Strodthoff, N.; Strodthoff, C. Detecting and interpreting myocardial infarction using fully convolutional neural networks. Physiol. Meas. 2019, 40, 015001. [CrossRef] [PubMed]

299. Hannun, A.Y.; Rajpurkar, P.; Haghpanahi, M.; Tison, G.H.; Bourn, C.; Turakhia, M.P.; Ng, A.Y. Cardiologist-level arrhythmia detection and classification in ambulatory electrocardiograms using a deep neural network. Nat. Med. 2019, 25, 65-69. [CrossRef] [PubMed]

300. Elul, Y.; Rosenberg, A.A.; Schuster, A.; Bronstein, A.M.; Yaniv, Y. Meeting the unmet needs of clinicians from AI systems showcased for cardiology with deep-learning-based ECG analysis. Proc. Natl. Acad. Sci. USA 2021, 118, 118. [CrossRef]

301. Attia, Z.I.; Kapa, S.; Lopez-Jimenez, F.; McKie, P.M.; Ladewig, D.J.; Satam, G.; Pellikka, P.A.; Enriquez-Sarano, M.; Noseworthy, P.A.; Munger, T.M.; et al. Screening for cardiac contractile dysfunction using an artificial intelligence-enabled electrocardiogram. Nat. Med. 2019, 25, 70-74. [CrossRef]

302. Pandey, A.; Kagiyama, N.; Yanamala, N.; Segar, M.W.; Cho, J.S.; Tokodi, M.; Sengupta, P.P. Deep-Learning Models for the Echocardiographic Assessment of Diastolic Dysfunction. JACC Cardiovasc. Imaging 2021, 14, 1887-1900. [CrossRef]

303. Ma, W.; Cheng, X.; Xu, X.; Wang, F.; Zhou, R.; Fenster, A.; Ding, M. Multilevel Strip Pooling-Based Convolutional Neural Network for the Classification of Carotid Plaque Echogenicity. Comput. Math. Methods Med. 2021, 2021, 3425893. [CrossRef]

304. Yang, Y.J.; Bang, C.S. Application of artificial intelligence in gastroenterology. World J. Gastroenterol. 2019, 25, 1666-1683. [CrossRef]

305. Namikawa, K.; Hirasawa, T.; Yoshio, T.; Fujisaki, J.; Ozawa, T.; Ishihara, S.; Aoki, T.; Yamada, A.; Koike, K.; Suzuki, H.; et al. Utilizing artificial intelligence in endoscopy: A clinician's guide. Expert Rev. Gastroenterol. Hepatol. 2020, 14, 689-706. [CrossRef] [PubMed]

306. Sung, J.J.; Poon, N.C. Artificial intelligence in gastroenterology: Where are we heading? Front. Med. 2020, 14, 511-517. [CrossRef]

307. Mori, Y.; Kudo, S.E.; Misawa, M.; Saito, Y.; Ikematsu, H.; Hotta, K.; Ohtsuka, K.; Urushibara, F.; Kataoka, S.; Ogawa, Y.; et al Real-Time Use of Artificial Intelligence in Identification of Diminutive Polyps During Colonoscopy: A Prospective Study. Ann. Intern. Med. 2018, 169, 357-366. [CrossRef] [PubMed]

308. Wang, P.; Xiao, X.; Glissen Brown, J.R.; Berzin, T.M.; Tu, M.; Xiong, F.; Hu, X.; Liu, P.; Song, Y.; Zhang, D.; et al. Development and validation of a deep-learning algorithm for the detection of polyps during colonoscopy. Nat. Biomed. Eng. 2018, 2, 741-748. [CrossRef] [PubMed]

309. Byrne, M.F.; Chapados, N.; Soudan, F.; Oertel, C.; Linares Perez, M.; Kelly, R.; Iqbal, N.; Chandelier, F.; Rex, D.K. Real-time differentiation of adenomatous and hyperplastic diminutive colorectal polyps during analysis of unaltered videos of standard colonoscopy using a deep learning model. Gut 2019, 68, 94-100. [CrossRef]

310. Urban, G.; Tripathi, P.; Alkayali, T.; Mittal, M.; Jalali, F.; Karnes, W.; Baldi, P. Deep Learning Localizes and Identifies Polyps in Real Time With 96\% Accuracy in Screening Colonoscopy. Gastroenterology 2018, 155, 1069-1078. [CrossRef]

311. Tokai, Y.; Yoshio, T.; Aoyama, K.; Horie, Y.; Yoshimizu, S.; Horiuchi, Y.; Ishiyama, A.; Tsuchida, T.; Hirasawa, T.; Sakakibara, Y.; et al. Application of artificial intelligence using convolutional neural networks in determining the invasion depth of esophageal squamous cell carcinoma. Esophagus 2020, 17, 250-256. [CrossRef]

312. Nakagawa, K.; Ishihara, R.; Aoyama, K.; Ohmori, M.; Nakahira, H.; Matsuura, N.; Shichijo, S.; Nishida, T.; Yamada, T.; Yamaguchi, S.; et al. Classification for invasion depth of esophageal squamous cell carcinoma using a deep neural network compared with experienced endoscopists. Gastrointest. Endosc. 2019, 90, 407-414. [CrossRef]

313. Ozawa, T.; Ishihara, S.; Fujishiro, M.; Saito, H.; Kumagai, Y.; Shichijo, S.; Aoyama, K.; Tada, T. Novel computer-assisted diagnosis system for endoscopic disease activity in patients with ulcerative colitis. Gastrointest. Endosc. 2019, 89, 416-421.e1. [CrossRef]

314. Mossotto, E.; Ashton, J.J.; Coelho, T.; Beattie, R.M.; MacArthur, B.D.; Ennis, S. Classification of Paediatric Inflammatory Bowel Disease using Machine Learning. Sci. Rep. 2017, 7, 2427. [CrossRef]

315. Molder, A.; Balaban, D.V.; Jinga, M.; Molder, C.C. Current Evidence on Computer-Aided Diagnosis of Celiac Disease: Systematic Review. Front. Pharm. 2020, 11, 341. [CrossRef]

316. Vecsei, A.; Amann, G.; Hegenbart, S.; Liedlgruber, M.; Uhl, A. Automated Marsh-like classification of celiac disease in children using local texture operators. Comput. Biol. Med. 2011, 41, 313-325. [CrossRef]

317. Wimmer, G.; Vecsei, A.; Uhl, A. CNN transfer learning for the automated diagnosis of celiac disease. In Proceedings of the 2016 Sixth International Conference on Image Processing Theory, Tools and Applications (IPTA) (IEEE 2016), Oulu, Finland, 12-15 December 2016; pp. 1-6. [CrossRef]

318. Hujoel, I.A.; Murphree, D.H., Jr.; Van Dyke, C.T.; Choung, R.S.; Sharma, A.; Murray, J.A.; Rubio-Tapia, A. Machine Learning in Detection of Undiagnosed Celiac Disease. Clin. Gastroenterol. Hepatol. 2018, 16, 1354-1355. [CrossRef] [PubMed]

319. Koh, J.E.W.; De Michele, S.; Sudarshan, V.K.; Jahmunah, V.; Ciaccio, E.J.; Ooi, C.P.; Gururajan, R.; Gururajan, R.; Oh, S.L.; Lewis, S.K.; et al. Automated interpretation of biopsy images for the detection of celiac disease using a machine learning approach. Comput. Methods Programs Biomed. 2021, 203, 106010. [CrossRef]

320. Klein, S.; Gildenblat, J.; Ihle, M.A.; Merkelbach-Bruse, S.; Noh, K.W.; Peifer, M.; Quaas, A.; Buttner, R. Deep learning for sensitive detection of Helicobacter Pylori in gastric biopsies. BMC Gastroenterol. 2020, 20, 417. [CrossRef] [PubMed] 
321. Gomolin, A.; Netchiporouk, E.; Gniadecki, R.; Litvinov, I.V. Artificial Intelligence Applications in Dermatology: Where Do We Stand? Front. Med. 2020, 7, 100. [CrossRef] [PubMed]

322. Spyridonos, P.; Gaitanis, G.; Likas, A.; Bassukas, I.D. Automatic discrimination of actinic keratoses from clinical photographs. Comput. Biol. Med. 2017, 88, 50-59. [CrossRef]

323. Hirota, M.; Kouzuki, H.; Ashikaga, T.; Sono, S.; Tsujita, K.; Sasa, H.; Aiba, S. Artificial neural network analysis of data from multiple in vitro assays for prediction of skin sensitization potency of chemicals. Toxicol. Vitr. 2013, 27, 1233-1246. [CrossRef]

324. Tsujita-Inoue, K.; Hirota, M.; Ashikaga, T.; Atobe, T.; Kouzuki, H.; Aiba, S. Skin sensitization risk assessment model using artificial neural network analysis of data from multiple in vitro assays. Toxicol. Vitr. 2014, 28, 626-639. [CrossRef] [PubMed]

325. Zang, Q.; Paris, M.; Lehmann, D.M.; Bell, S.; Kleinstreuer, N.; Allen, D.; Matheson, J.; Jacobs, A.; Casey, W.; Strickland, J. Prediction of skin sensitization potency using machine learning approaches. J. Appl. Toxicol. 2017, 37, 792-805. [CrossRef]

326. Esteva, A.; Kuprel, B.; Novoa, R.A.; Ko, J.; Swetter, S.M.; Blau, H.M.; Thrun, S. Dermatologist-level classification of skin cancer with deep neural networks. Nature 2017, 542, 115-118. [CrossRef]

327. Haenssle, H.A.; Fink, C.; Schneiderbauer, R.; Toberer, F.; Buhl, T.; Blum, A.; Kalloo, A.; Hassen, A.B.H.; Thomas, L.; Enk, A.; et al. Man against machine: Diagnostic performance of a deep learning convolutional neural network for dermoscopic melanoma recognition in comparison to 58 dermatologists. Ann. Oncol. 2018, 29, 1836-1842. [CrossRef]

328. Haenssle, H.A.; Fink, C.; Toberer, F.; Winkler, J.; Stolz, W.; Deinlein, T.; Hofmann-Wellenhof, R.; Lallas, A.; Emmert, S.; Buhl, T.; et al. Man against machine reloaded: Performance of a market-approved convolutional neural network in classifying a broad spectrum of skin lesions in comparison with 96 dermatologists working under less artificial conditions. Ann. Oncol. 2020, 31, 137-143. [CrossRef] [PubMed]

329. Han, S.S.; Kim, M.S.; Lim, W.; Park, G.H.; Park, I.; Chang, S.E. Classification of the Clinical Images for Benign and Malignant Cutaneous Tumors Using a Deep Learning Algorithm. J. Investig. Derm. 2018, 138, 1529-1538. [CrossRef]

330. Yap, J.; Yolland, W.; Tschandl, P. Multimodal skin lesion classification using deep learning. Exp. Derm. 2018, 27, 1261-1267. [CrossRef]

331. Phillips, M.; Marsden, H.; Jaffe, W.; Matin, R.N.; Wali, G.N.; Greenhalgh, J.; McGrath, E.; James, R.; Ladoyanni, E.; Bewley, A.; et al. Assessment of Accuracy of an Artificial Intelligence Algorithm to Detect Melanoma in Images of Skin Lesions. JAMA Netw. Open 2019, 2, e1913436. [CrossRef] [PubMed]

332. Kharazmi, P.; Kalia, S.; Lui, H.; Wang, Z.J.; Lee, T.K. A feature fusion system for basal cell carcinoma detection through data-driven feature learning and patient profile. Ski. Res. Technol. 2018, 24, 256-264. [CrossRef]

333. Hekler, A.; Utikal, J.S.; Enk, A.H.; Hauschild, A.; Weichenthal, M.; Maron, R.C.; Berking, C.; Haferkamp, S.; Klode, J.; Schadendorf, D.; et al. Superior skin cancer classification by the combination of human and artificial intelligence. Eur. J. Cancer 2019, 120, 114-121. [CrossRef]

334. Brinker, T.J.; Hekler, A.; Enk, A.H.; Berking, C.; Haferkamp, S.; Hauschild, A.; Weichenthal, M.; Klode, J.; Schadendorf, D.; Holland-Letz, T.; et al. Deep neural networks are superior to dermatologists in melanoma image classification. Eur. J. Cancer 2019, 119, 11-17. [CrossRef] [PubMed]

335. Brinker, T.J.; Hekler, A.; Enk, A.H.; Klode, J.; Hauschild, A.; Berking, C.; Schilling, B.; Haferkamp, S.; Schadendorf, D.; Holland-Letz, T.; et al. Deep learning outperformed 136 of 157 dermatologists in a head-to-head dermoscopic melanoma image classification task. Eur. J. Cancer 2019, 113, 47-54. [CrossRef] [PubMed]

336. Tschandl, P.; Rosendahl, C.; Akay, B.N.; Argenziano, G.; Blum, A.; Braun, R.P.; Cabo, H.; Gourhant, J.Y.; Kreusch, J.; Lallas, A.; et al. Expert-Level Diagnosis of Nonpigmented Skin Cancer by Combined Convolutional Neural Networks. JAMA Derm. 2019, 155, 58-65. [CrossRef]

337. Arevalo, J.; Cruz-Roa, A.; Arias, V.; Romero, E.; Gonzalez, F.A. An unsupervised feature learning framework for basal cell carcinoma image analysis. Artif. Intell. Med. 2015, 64, 131-145. [CrossRef]

338. De Guzman, L.C.; Maglaque, R.P.C.; Torres, V.M.B.; Zapido, S.P.A.; Cordel, M.O. Design and Evaluation of a Multi-model, Multi-level Artificial Neural Network for Eczema Skin Lesion Detection. In Proceedings of the 2015 3rd International Conference on Artificial Intelligence, Modelling and Simulation (AIMS), Kota Kinabalu, Malaysia, 2-4 December 2015; pp. 42-47. [CrossRef]

339. Han, S.S.; Park, G.H.; Lim, W.; Kim, M.S.; Na, J.I.; Park, I.; Chang, S.E. Deep neural networks show an equivalent and often superior performance to dermatologists in onychomycosis diagnosis: Automatic construction of onychomycosis datasets by region-based convolutional deep neural network. PLoS ONE 2018, 13, e0191493. [CrossRef]

340. Binol, H.; Plotner, A.; Sopkovich, J.; Kaffenberger, B.; Niazi, M.K.K.; Gurcan, M.N. Ros-NET: A deep convolutional neural network for automatic identification of rosacea lesions. Ski. Res. Technol. 2020, 26, 413-421. [CrossRef]

341. Meienberger, N.; Anzengruber, F.; Amruthalingam, L.; Christen, R.; Koller, T.; Maul, J.T.; Pouly, M.; Djamei, V.; Navarini, A.A. Observer-independent assessment of psoriasis-affected area using machine learning. J. Eur. Acad. Derm. Venereol. 2020, 34, 1362-1368. [CrossRef] [PubMed]

342. Fadzil, M.H.; Ihtatho, D.; Affandi, A.M.; Hussein, S.H. Area assessment of psoriasis lesions for PASI scoring. J. Med. Eng. Technol. 2009, 33, 426-436. [CrossRef]

343. George, Y.; Aldeen, M.; Garnavi, R. Automatic Scale Severity Assessment Method in Psoriasis Skin Images Using Local Descriptors. IEEE J. Biomed. Health Inf. 2020, 24, 577-585. [CrossRef] [PubMed]

344. Pal, A.; Garain, U.; Chandra, A.; Chatterjee, R.; Senapati, S. Psoriasis skin biopsy image segmentation using Deep Convolutional Neural Network. Comput. Methods Programs Biomed. 2018, 159, 59-69. [CrossRef] [PubMed] 